Article

\title{
On Multivariate Bernstein Polynomials
}

\author{
Mama Foupouagnigni ${ }^{1,2, \dagger}$ and Merlin Mouafo Wouodjié ${ }^{3, *, \dagger}$ \\ 1 African Institute for Mathematical Sciences, P.O. Box 608, Limbe, Cameroon; \\ mfoupouagnigni@aims-cameroon.org \\ 2 Higher Teacher Training College, University of Yaounde I, P.O. Box 47, Yaounde, Cameroon \\ 3 Institute of Mathematics, University of Kassel, Heinrich-Plett Str. 40, 34132 Kassel, Germany \\ * Correspondence: merlin@mathematik.uni-kassel.de \\ + These authors contributed equally to this work.
}

Received: 20 June 2020; Accepted: 27 July 2020; Published: 21 August 2020

check for updates

\begin{abstract}
In this paper, we first revisit and bring together as a sort of survey, properties of Bernstein polynomials of one variable. Secondly, we extend the results from one variable to several ones, namely-uniform convergence, uniform convergence of the derivatives, order of convergence, monotonicity, fixed sign for the $p$-th derivative, and deduction of the upper and lower bounds of Bernstein polynomials from those of the corresponding functions.
\end{abstract}

Keywords: Bernstein polynomials; functions of several variables; derivatives; uniform convergence; order of convergence; monotonicity; convexity; upper and lower bounds

\section{Introduction}

It is not often easy to find exact solutions to most equations governing real-life problems. Even when such solutions can be found, for their practical use, there is a need to find their approximation by polynomials, when possible.

Weierstrass's theorem asserts the possibility of uniform approximation by polynomials of continuous functions over a closed interval. An analytic function can be expanded in a uniformly convergent power series and a continuous but non-analytic function can be expanded in a uniformly convergent series of general polynomials, with no possibility of rearranging its terms so as to produce a convergent power series [1]. There are many proofs of the Weierstrass theorem. In this paper, we shall present one called "S. Bernstein's proof" since it also gives an explicit representation of the polynomials for uniform approximation. An example of Bernstein polynomials of one variable is (see Reference [1]):

$$
B_{n}(f ; x)=\sum_{k=0}^{n} f\left(\frac{k}{n}\right)\left(\begin{array}{l}
n \\
k
\end{array}\right) x^{k}(1-x)^{n-k} .
$$

The polynomials $B_{n}(f ;$.$) are known to approximate uniformly their corresponding functions f$ in $[0,1]$, provided that $f$ is continuous on the interval $[0,1]$. Moreover, the $n^{\text {th }}$ derivative of $B_{n}(f ;$.$) converges$ uniformly to $f^{(n)}$, provided that $f \in \mathcal{C}^{n}[0,1]\left(f \in \mathcal{C}^{n}[0,1]\right.$ means $f$ is continuous on the interval $[0,1]$ and all its derivatives $f^{(i)}, i=1, \cdots, n$, exist and are also continuous on $\left.[0,1]\right)$. According to the literature, there are several ways (with different notations) to define explicitly the Bernstein polynomials of one variable, which lead to the same results as in Reference [1] - uniform convergence as well as uniform convergence of the derivatives (see References [2-7]). 
Bernstein polynomials have been used for approximation of functions in many areas of Mathematics and other fields such as smoothing in Statistics, and constructing and generalizing Bézier curves (see References [8,9]). We also have some applications:

1. in the quadrature rule on equally spaced knots (see Reference [10]);

2. to numerical differentiation and quadrature and perform numerical experiments showing the effectiveness of the considered technique (see Reference [11]): they perform some numerical tests for approximation by polynomials of a given function, numerical differentiation and numerical quadrature.

"In contrast to other modes of approximation, in particular to Tchebyschev or best uniform approximation, the Bernstein polynomials yield smooth approximants; but there is a price that must be paid for these beautiful approximations properties: the convergence of the Bernstein polynomials is very slow."

P. J. Davis [1].

Several authors like A. Jafarian, S. Measoomy Nia, Alireza K. Golmankhaneh and D. Baleanu [12], D. H. Tucker [13], E. H. Doha, A. H. Bhrawy, M. A. Saker [14], George M. Phillips [15], Henryk Gzyl and José Luis Palacios [16], M. H. T Alshbool, I. Hashim [17], P. J. Davis [1], Richard V. Kadison [18] and others, have worked on Bernstein polynomials for functions with one variable, and have derived some beautiful properties whose most important ones are: the uniform convergence, the uniform convergence of derivatives, the fixed sign for the $p^{\text {th }}$ derivative, the deduction of upper and lower bounds of Bernstein polynomials from those of the corresponding functions.

The main problem here is to extend these properties of Bernstein polynomials of one variable by generalizing them for functions of several real variables. There are more than five ways with different notations to define Bernstein polynomials in the multi-dimensional case for a function $f$ defined on $[0,1]^{m}$, where $m$ is the dimension of the space. Some used notations are $x=\left(x_{1}, \cdots, x_{m}\right) \in[0,1]^{m}$, $k=\left(k_{1}, \cdots, k_{m}\right) \in \mathbb{N}^{m},|x|=x_{1}+\cdots+x_{m}, x^{k}=x_{1}^{k_{1}} \cdots x_{m}^{k_{m}},|k|=k_{1}+\cdots+k_{m}, k !=k_{1} ! \cdots k_{m}$ ! and $\frac{k}{n}=\left(\frac{k_{1}}{n}, \cdots, \frac{k_{m}}{n}\right), n \in \mathbb{N} \backslash\{0\}$. These notations are used in the following definitions:

(a) the Bernstein polynomials on $(m-1)$-dimensional simplex in cube $[0,1]^{m}$ (see References [19-22]):

$$
b^{n}[f](x):=\sum_{|k|=n} f\left(\frac{k}{n}\right) \frac{n !}{k !} x^{k}, \quad \text { where } x_{1}+\cdots+x_{m}=1
$$

(b) the Bernstein polynomials on a $m$-dimensional simplex and very useful in stochastic analysis (see References [7,23-26]):

$$
B_{n, m}(f, x):=\sum_{|k| \leq n} f\left(\frac{k}{n}\right) \frac{n !}{k !(n-|k|) !} x^{k}(1-|x|)^{n-|k|}, \quad \text { where } \quad x_{1}+\cdots+x_{m} \leq 1 ;
$$

(c) the Bernstein polynomials on simplices with a pointwise orthogonality relation (see Reference [27]):

$$
B_{n}(f, x):=\sum_{|\alpha|=n} f\left(\frac{\bar{\alpha}}{n}\right) \frac{(|\alpha|) !}{\alpha !} x^{\bar{\alpha}}(1-|x|)^{\alpha_{m+1}},
$$

where $\alpha=\left(\alpha_{1}, \cdots, \alpha_{m+1}\right) \in \mathbb{N}^{m+1}$ and $\bar{\alpha}=\left(\alpha_{1}, \cdots, \alpha_{m}\right)$; 
(d) the Bernstein polynomials on $(m-1)$-dimensional simplex in cube $[0,1]^{m}$ used in the field of mathematical biology with quadrartic mappings of "standard" $(m-1)$-dimensional simplex to self (see Reference [21]):

$$
B_{n}(f)\left(x_{1}, \cdots, x_{m}\right)=\sum_{k_{1}=0}^{n} \cdots \sum_{k_{m}=0}^{n} f\left(\frac{k}{n}\right) \prod_{i=1}^{m}\left(\begin{array}{l}
n \\
k_{i}
\end{array}\right) x_{i}^{k_{i}}\left(1-x_{i}\right)^{n-k_{i}} ;
$$

(e) the Bernstein polynomials related to the convergence in the uniform and $L_{p}$-norms for polynomials with integral coefficients (see Reference [28] for two variables, References [1,29] for the generalized one):

$$
B_{n_{1}, \cdots, n_{m}}\left(f ; x_{1}, \cdots, x_{m}\right)=\sum_{k_{1}=0}^{n_{1}} \cdots \sum_{k_{m}=0}^{n_{m}} f\left(\frac{k_{1}}{n_{1}}, \cdots, \frac{k_{m}}{n_{m}}\right) \prod_{i=1}^{m}\left(\begin{array}{l}
n_{i} \\
k_{i}
\end{array}\right) x_{i}^{k_{i}}\left(1-x_{i}\right)^{n_{i}-k_{i}},
$$

where $n_{1}, \cdots, n_{m} \in \mathbb{N}$.

Using the above mentioned definitions, many authors did some investigations aimed at generalizing some properties of univariate Bernstein polynomials to Bernstein polynomials with two or more variables. Let us take for example the forms given by (2), (3) and (6).

1. For the multivariate Bernstein polynomials given by Equation (2): they have proven the pointwise and uniform convergence (see References $[21,22,30]$ ). They have also presented two variations which are preserved and do coincide with classical convexity in the univariate case (see Reference [31]). Moreover, they showed the monotonicity of successive Bernstein polynomials for classically convex functions (see References [31,32]).

2. For the multivariate Bernstein polynomials given by Equation (3): they have proven two identities for these polynomials on simplex, which are considered on a pointwise (see References [23-25,25]). They also showed that these polynomials converge to their corresponding functions $f$ along with partial derivatives provided that the latter derivatives exist and are continuous (see References [7,26,33]).

3. For the Bernstein polynomials given by Equation (6): they established (but only for two variables) the pointwise and uniform convergence; the same result was established for the partial derivatives of the corresponding function (see References [28,29,34-36]).

According to the last definition (see Equation (6)) of the generalized Bernstein polynomial $B_{n_{1}, \cdots, n_{m}}\left(f ; x_{1}, \cdots, x_{m}\right)$, there is just a few new results (pointwise and uniform convergence) given by some authors like Clemens Heitzinger (see Reference [29]). That is why we focus, in this paper, on the multivariate Bernstein polynomials given by the form defined by Equation (6).

Many authors have made tremendous efforts to prove some properties for multivariate Bernstein polynomials on simplex (see Reference [23]), which are considered on a pointwise convergence (good approximations of Bernstein polynomials for every continuous function on simplex and the higher dimensional q-analogue of Bernstein polynomials on simplex). Some have tried to study the approximation by combination of Bernstein polynomials or operators (the rate of convergence of derivatives and the smoothness of the derivatives of functions) in order to approximate, under definite conditions, the function more closely than the Bernstein polynomials (see References [3,6,37-41]). Moreover, they defined and also tried to study the q-Bernstein polynomials (see References [2,4,42-45]), the generalized Bernstein polynomials (see References $[10,26]$ ) and the modified Bernstein polynomials (see Reference [25]).

To achieve our aim which is to generalize these beautiful properties for functions with one variable to functions with several variables, we will use the following properties of univariate Bernstein polynomials (with some references where they can be found): 
- Property 1 (see Reference [1]): if $f$ is bounded on $[0,1]$ and continuous at $x_{0} \in[0,1]$, then

$$
\lim _{n \rightarrow \infty} B_{n}\left(f ; x_{0}\right)=f\left(x_{0}\right) ;
$$

- $\quad$ Property 2 (see References $[1,3,5-7,10,11,39,46-48])$ : if $f \in \mathcal{C}[0,1]$, the Bernstein polynomial $B_{n}(f ; \cdot)$ converges uniformly to $f$;

- Property 3 (see References $[1,7,11]$ ): if $f \in \mathcal{C}^{p}[0,1]$ ( $f$ is continuous on the interval $[0,1]$ and all its derivatives $f^{(i)}, i=1, \cdots, p$, exist and are also continuous on $\left.[0,1]\right)$, then $B_{n}^{p}\left(f_{;} \cdot\right)$ converges uniformly to $f^{(p)}$;

- Property 4 (see References [1,21]): if $f \in \mathcal{C}^{p}[0,1]$, for some $0 \leq p \leq n$ and $A \leq f^{(p)}(x) \leq$ $B$ (A and $\mathrm{B}$ are constants $), 0 \leq x \leq 1$, then

$$
A \leq \frac{n^{p}}{n(n-1) \cdots(n-p+1)} B_{n}^{(p)}(f ; x) \leq B, \quad 0 \leq x \leq 1 ;
$$

(the consequence is: if $f$ is decreasing (resp. nondecreasing) on $[0,1]$, then $B_{n}(f ; \cdot)$ is decreasing (resp. nondecreasing) on $[0,1]$.)

- Property 5 (see References [1,31]): if $f$ is convex in $[0,1]$, then $B_{n}(f ; \cdot)$ is convex there and for $n=2,3, \cdots$

$$
B_{n-1}(f ; x) \geq B_{n}(f ; x), 0<x<1 ;
$$

- Property 6 (see References [1,11,39]): if $f(x)$ is bounded on $[0,1]$ and $x_{0}$ is a point of $[0,1]$ at which $f^{\prime \prime}$ exists and is continuous, then

$$
\lim _{n \rightarrow \infty} n\left[B_{n}\left(f ; x_{0}\right)-f\left(x_{0}\right)\right]=\frac{1}{2} x_{0}\left(1-x_{0}\right) f^{\prime \prime}\left(x_{0}\right) .
$$

The following intermediate results for the case of univariate Bernstein polynomials are needed to prove all those properties:

(a) see References [1,15]: for $x \in[0,1]$ we have $B_{n}(1 ; x)=1$;

(b) see Reference [1]: for $x \in[0,1]$ and $\alpha \in \mathbb{R}$ we have

$$
B_{n}(\exp (\alpha x) ; x)=(x+\exp (\alpha / n)+(1-x))^{n} ;
$$

(c) see References [1,15]: the Bernstein polynomial may be expressed in the form

$$
B_{n}(f ; x)=\sum_{k=0}^{n}\left(\begin{array}{l}
n \\
k
\end{array}\right) \triangle^{k} f_{n}(0) x^{k},
$$

where $f_{n}(x)=f\left(\frac{x}{n}\right)$ and $\triangle$ is the forward difference operator with step size $h=\frac{1}{n}$ defined as follows (see Reference [15]):

$$
\left\{\begin{array}{l}
\triangle f\left(x_{i}\right):=f\left(x_{i+1}\right)-f\left(x_{i}\right) \\
\triangle^{k+1} f\left(x_{i}\right):=\triangle\left(\triangle^{k} f\left(x_{i}\right)\right)=\triangle^{k} f\left(x_{i+1}\right)-\triangle^{k} f\left(x_{i}\right) \text { fork } \geq 1 \\
\triangle^{1} f\left(x_{i}\right):=\triangle f\left(x_{i}\right) \text { and } \triangle^{0} f\left(x_{i}\right):=f\left(x_{i}\right)
\end{array}\right.
$$

where $\triangle^{k}$ is called the $k^{\text {th }}$ difference; 
(d) See References $[1,15,21,22]$ : for a given $\delta>0, n \in \mathbb{N} \backslash\{0\}$ and $x \in[0,1]$, we have

$$
\sum_{\left|\frac{k}{n}-x\right| \geq \delta}\left(\begin{array}{l}
n \\
k
\end{array}\right) x^{k}(1-x)^{n-k} \leq \frac{1}{4 n \delta^{2}}
$$

(e) see References [1,15]: for any integer $k \geq 0$, the $k$ - th derivative of $B_{n+k}(f ; x)$ may be expressed in terms of $k$-th differences of $f$ as

$$
B_{n+k}^{(k)}(f ; x)=\frac{(n+k) !}{n !} \sum_{r=0}^{n} \Delta^{k} f\left(\frac{r}{n+k}\right)\left(\begin{array}{l}
n \\
r
\end{array}\right) x^{r}(1-x)^{n-r}
$$

for all $n \geq 0$, where $\triangle$ is applied with step size $h=\frac{1}{n+k}$;

(f) see (Reference [1] Lemma 6.3.5): there is a constant $C$ independent of a given positive integer $n$ such that for all $x$ in $[0,1]$

$$
\sum_{\left|\frac{k}{n}-x\right| \geq n^{-1 / 4}}\left(\begin{array}{l}
n \\
k
\end{array}\right) x^{k}(1-x)^{n-k} \leq \frac{C}{n^{3 / 2}} .
$$

With all those properties, we will prove the following for our generalized Bernstein polynomials:

- Property 1: if $f$ is bounded on $[0,1]^{m}$ and continuous on $\left(a_{1}, \cdots, a_{m}\right) \in[0,1]^{m}$, then the Bernstein polynomial $B_{n_{1}, \cdots, n_{m}}\left(f ; a_{1}, \cdots, a_{m}\right)$ converges to $f\left(a_{1}, \cdots, a_{m}\right)$, as $n_{i} \rightarrow \infty$ for all $i$;

- Property 2: if $f \in \mathcal{C}[0,1]^{m}$, then the Bernstein polynomial $B_{n_{1}, \cdots, n_{m}}(f ; \cdot)$ converges uniformly to $f$;

- Property 3: if $f \in \mathcal{C}^{p_{1} \cdots p_{m}}[0,1]^{m}$, then $B_{n_{1}, \cdots, n_{m}}^{p_{1}, \cdots, p_{m}}(f ; \cdot)$ converges uniformly to $f^{\left(p_{1}\right), \cdots,\left(p_{m}\right)}$, where $f \in$ $\mathcal{C}^{p_{1}, \cdots, p_{m}}[0,1]^{m}$ means $f$ is continuous on $[0,1]^{m}$ and $\frac{\partial^{p_{i}} f}{\partial x^{p_{i}}}$ is continuous on $[0,1]^{m}, 0 \leq p_{i} \leq n_{i}$, $i=1, \cdots, m$;

- $\quad$ Property 4: if $f \in \mathcal{C}^{p_{1} \cdots p_{m}}[0,1]^{m}, 0 \leq p_{i} \leq n_{i}, i=1, \cdots, m$, and $A \leq f^{\left(p_{1}\right) \cdots\left(p_{m}\right)}\left(x_{1}, \cdots, x_{m}\right) \leq B$, $\left(x_{1}, \cdots, x_{m}\right) \in[0,1]^{m}$, then

$$
A \leq \prod_{i=1}^{m} \frac{n_{i}^{p_{i}}}{n_{i}\left(n_{i}-1\right) \cdots\left(n_{i}-p_{i}+1\right)} B_{n_{1}, \cdots, n_{m}}^{p_{1}, \cdots, p_{m}}\left(f ; x_{1}, \cdots, x_{m}\right) \leq B
$$

for all $\left(x_{1}, \cdots, x_{m}\right) \in[0,1]^{m}$. As a consequence, if $f\left(x_{1}, \cdots, x_{m}\right)$ is decreasing (resp. nondecreasing) on $[0,1]$ with respect to the variable $x_{i}, i \in 1, \cdots, m$, then $B_{n_{1}, \cdots, n_{m}}\left(f ; x_{1}, \cdots, x_{m}\right)$ is decreasing (resp. nondecreasing) on $[0,1]$ with respect to the variable $x_{i}$ there.

- Property 5: if $f\left(x_{1}, \cdots, x_{m}\right)$ is convex in $[0,1]^{m}$, then, for $n_{i}=2,3, \cdots$ with $i=1, \cdots, m$, we have

$$
B_{n_{1}-1, \cdots, n_{m}-1}\left(f ; x_{1}, \cdots, x_{m}\right) \geq B_{n_{1}, \cdots, n_{m}}\left(f ; x_{1}, \cdots, x_{m}\right)
$$

for all $\left(x_{1}, \cdots, x_{m}\right) \in[0,1]^{m}$;

- Property 6: if $f\left(x_{1}, \cdots, x_{m}\right)$ is bounded in $[0,1]^{m}$ and let $\left(a_{1}, \cdots, a_{m}\right)$ be a point of $[0,1]^{m}$ at which $f^{(2) \cdots(2)}\left(x_{1}, \cdots, x_{m}\right)$ exists and is continuous, then, for $n_{1}=n_{2}=\cdots=n_{m}=n$

$$
\lim _{n \rightarrow \infty} n\left[B_{n, \cdots, n}\left(f ; a_{1}, \cdots, a_{m}\right)-f\left(a_{1}, \cdots, a_{m}\right)\right]=\frac{1}{2} \sum_{i=1}^{m} a_{i}\left(1-a_{i}\right) \frac{\partial^{2} f}{\partial x_{i}^{2}}\left(a_{1}, \cdots, a_{m}\right) .
$$


The following intermediate results for the multivariate case, that we will also prove, are needed in order to prove all these cited properties:

(a) for $m \geq 1, i \in\{1, \cdots, m\}, j_{i}$ and $n_{i}$ integers, $n_{i} \geq 1$, and $x_{i} \in[0,1]$

$$
\left\{\begin{array}{l}
x_{1}^{j_{1}} \cdots x_{m}^{j_{m}}=\sum_{k_{1}=0}^{j_{1}} \cdots \sum_{k_{m}=0}^{j_{m}} \prod_{i=1}^{m} \sigma_{j_{i}, k_{i}}\left(\begin{array}{c}
n_{i} \\
k_{i}
\end{array}\right) x_{i}^{k_{i}}\left(1-x_{i}\right)^{n_{i}-k_{i}}, \\
B_{n_{1}, \cdots, n_{m}}\left(1 ; x_{1}, \cdots, x_{m}\right)=1 \\
B_{n_{1}, \cdots, n_{m}}\left(\prod_{i=1}^{m} x_{i}^{j_{i}} ; x_{1}, \cdots, x_{m}\right)=\prod_{i=1}^{m} B_{n_{i}}\left(x_{i}^{j_{i}} ; x_{i}\right),
\end{array}\right.
$$

where $\sigma_{j_{i}, k_{i}}$ are real numbers;

(b) for $i \in\{1, \cdots, m\}, x_{i} \in[0,1]$ and $\alpha_{i} \in \mathbb{R}$

$$
\begin{aligned}
& B_{n_{1}, \cdots, n_{m}}\left(\exp \left(\sum_{i=1}^{m} \alpha_{i} x_{i}\right) ; x_{1}, \cdots, x_{m}\right)=\prod_{i=1}^{m} B_{n_{i}}\left(\exp \left(\alpha_{i} x_{i}\right) ; x_{i}\right)=\prod_{i=1}^{m}\left[x_{i} \exp \left(\alpha_{i} / n_{i}\right)+\left(1-x_{i}\right)\right]^{n_{i}}, \\
& \sum_{k_{1}=0}^{n_{1}} \cdots \sum_{k_{m}=0}^{n_{m}} \prod_{i=1}^{m}\left(\begin{array}{c}
n_{i} \\
k_{i}
\end{array}\right)\left(\frac{k_{i}}{n_{i}}-x_{i}\right)^{j_{i}} x_{i}^{k_{i}}\left(1-x_{i}\right)^{n_{i}-k_{i}}=\prod_{i=1}^{m} \sum_{k_{i}=0}^{n_{i}}\left(\begin{array}{c}
n_{i} \\
k_{i}
\end{array}\right)\left(\frac{k_{i}}{n_{i}}-x_{i}\right)^{j_{i}} x_{i}^{k_{i}}\left(1-x_{i}\right)^{n_{i}-k_{i}} ;
\end{aligned}
$$

(c) the Bernstein polynomial may be expressed in the form

$$
B_{n_{1}, \cdots, n_{m}}\left(f ; x_{1}, \cdots, x_{m}\right)=\sum_{t_{1}=0}^{n_{1}} \cdots \sum_{t_{m}=0}^{n_{m}} \triangle_{1}^{t_{1}} \cdots \triangle_{m}^{t_{m}} f_{n_{1}, \cdots, n_{m}}(0, \cdots, 0) \prod_{i=1}^{m}\left(\begin{array}{c}
n_{i} \\
t_{i}
\end{array}\right) x_{i}^{t_{i}}
$$

where $f_{n_{1}, \cdots, n_{m}}\left(t_{1}, \cdots, t_{m}\right)=f\left(\frac{t_{1}}{n_{1}}, \cdots, \frac{t_{m}}{n_{m}}\right)$ and $\triangle_{i}^{t_{i}}, i \in\{1, \cdots, m\}$, is the $t_{i}^{\text {th }}$ difference(s) related to the $i^{\text {th }}$ variable $x_{i}$, and applied with step size $h_{i}=\frac{1}{n_{i}}$.

(d) for a given $\delta_{i}>0, i=1, \cdots, m$ and $\left(x_{1}, \cdots, x_{m}\right) \in[0,1]^{m}$, we have

$$
\sum_{\left|\frac{k_{1}}{n_{1}}-x_{1}\right| \geq \delta_{1}} \ldots \sum_{\left|\frac{k_{m}}{n_{m}}-x_{m}\right| \geq \delta_{m}} \prod_{i=1}^{m}\left(\begin{array}{c}
n_{i} \\
k_{i}
\end{array}\right) x_{i}^{k_{i}}\left(1-x_{i}\right)^{n_{i}-k_{i}} \leq \frac{1}{4^{m} \prod_{i=1}^{m} n_{i} \delta_{i}^{2}}
$$

summing over those values of $k_{i}=0, \cdots, n_{i}, i=1 \cdots m$, for which $\left|\frac{k_{i}}{n_{i}}-x_{i}\right| \geq \delta_{i}$;

(e) for $p_{1}, \cdots, p_{m} \geq 0$ integers with $0 \leq p_{i} \leq n_{i}, i=1,2, \cdots, m$, the $\left(p_{1}, \cdots, p_{m}\right)^{\text {th }}$ derivative of $B_{n_{1}+p_{1}, \cdots, n_{m}+p_{m}}\left(f ; x_{1}, \cdots, x_{m}\right)$ may be expressed in terms of $\left(p_{1}, \cdots, p_{m}\right)^{\text {th }}$ differences of $f$ as

$$
\begin{aligned}
& B_{n_{1}+p_{1}, \cdots, n_{m}+p_{m}}^{p_{1}, \cdots, p_{m}}\left(f ; x_{1}, \cdots, x_{m}\right) \\
= & \left(\prod_{i=1}^{m} \frac{\left(n_{i}+p_{i}\right) !}{n_{i} !}\right) \sum_{t_{1}=0}^{n_{1}} \cdots \sum_{t_{m}=0}^{n_{m}} \triangle_{1}^{p_{1}} \cdots \triangle_{m}^{p_{m}} f\left(\frac{t_{1}}{n_{1}+p_{1}}, \cdots, \frac{t_{m}}{n_{m}+p_{m}}\right) \prod_{i=1}^{m}\left(\begin{array}{c}
n_{i} \\
t_{i}
\end{array}\right) x_{i}^{t_{i}}\left(1-x_{i}\right)^{n_{i}-t_{i}},
\end{aligned}
$$

where the $\Delta_{k}$ is acting with the mesh $h_{k}=\frac{1}{n_{k}+p_{k}}$;

(f) there is a constant $C$ independent of given positive integers $n_{i}$ such that for all $\left(x_{1}, \cdots, x_{m}\right) \in$ $[0,1]^{m}, i=1, \cdots, m$, 


$$
\sum_{\left|\frac{k_{1}}{n_{1}}-x_{1}\right| \geq n_{1}^{-1 / 4}} \cdots \sum_{\left|\frac{k_{m}}{n_{m}}-x_{m}\right| \geq n_{m}^{-1 / 4}} \prod_{i=1}^{m}\left(\begin{array}{l}
n_{i} \\
k_{i}
\end{array}\right) x_{i}^{k_{i}}\left(1-x_{i}\right)^{n_{i}-k_{i}} \leq \frac{C}{\prod_{i=1}^{m} n_{i}^{3 / 2}}
$$

\section{Definition and Properties}

We shall now derive the properties of the $m$-dimensional generalization of the Bernstein polynomials. First of all, we give $m$-dimensional generalization of the Bernstein polynomials.

Definition 1 (Generalization). (P. J. Davis [1], page 122)

Let $f\left(x_{1}, \cdots, x_{m}\right)$ be defined on $[0,1]^{m}$ and $n_{i} \geq 1, i=1, \cdots, m$, integers. The $\left(n_{1}, \cdots, n_{m}\right)^{\text {th }}$ Bernstein polynomial for $f\left(x_{1}, \cdots, x_{m}\right)$ is given by

$$
B_{n_{1}, \cdots, n_{m}}\left(f ; x_{1}, \cdots, x_{m}\right)=\sum_{k_{1}=0}^{n_{1}} \cdots \sum_{k_{m}=0}^{n_{m}} f\left(\frac{k_{1}}{n_{1}}, \cdots, \frac{k_{m}}{n_{m}}\right) \prod_{i=1}^{m} b_{k_{i}, n_{i}}\left(x_{i}\right),
$$

where $b_{k_{i}, n_{i}}\left(x_{i}\right)=\left(\begin{array}{l}n_{i} \\ k_{i}\end{array}\right) x_{i}^{k_{i}}\left(1-x_{i}\right)^{n_{i}-k_{i}}, \quad i=1, \cdots, m$.

Notice that

$$
\left\{\begin{array}{l}
B_{n_{1}, \cdots, n_{m}}(f ; 0, \cdots, 0)=f(0, \cdots, 0) ; \\
B_{n_{1}, \cdots, n_{m}}(f ; 1, \cdots, 1)=f(1, \cdots, 1) .
\end{array}\right.
$$

We call $B_{n_{1}, \cdots, n_{m}}$ the Bernstein operator; it maps a function $f$, defined on $[0,1]^{m}$, to $B_{n_{1}, \cdots, n_{m}} f$, where the function $B_{n_{1}, \cdots, n_{m}} f$ evaluated at $\left(x_{1}, \cdots, x_{m}\right)$ is denoted by $B_{n_{1}, \cdots, n_{m}}\left(f ; x_{1}, \cdots, x_{m}\right)$. This operator is linear, since it follows from Equation (27) that

$$
B_{n_{1}, \cdots, n_{m}}(\lambda f+\mu g)=\lambda B_{n_{1}, \cdots, n_{m}} f+\mu B_{n_{1}, \cdots, n_{m}} g,
$$

for all functions $f$ and $g$ defined on $[0,1]^{m}$, and all real $\lambda$ and $\mu$.

Using the results obtained with one variable, it is easy to obtain some pertinent properties for the generalization of Bernstein polynomials.

\section{Lemma 1.}

1 Let $m \geq 1, j_{1}, \cdots, j_{m}$ be integers. Then,

$$
x_{1}^{j_{1}} \cdots x_{m}^{j_{m}}=\sum_{k_{1}=0}^{j_{1}} \cdots \sum_{k_{m}=0}^{j_{m}} \prod_{i=1}^{m} \sigma_{j_{i}, k_{i}} b_{k_{i}, j_{i}}\left(x_{i}\right),
$$

where $\sigma_{j_{i}, k_{i}}, k_{i}=0, \cdots, j_{i}, 1 \leq i \leq m$, are real numbers.

2 For $i \in\{1, \cdots, m\}$, let $n_{i} \geq 1, j_{i}$ be integer and $x_{i} \in[0,1]$. Then,

$$
\left\{\begin{array}{l}
B_{n_{1}, \cdots, n_{m}}\left(1 ; x_{1}, \cdots, x_{m}\right)=1 \\
B_{n_{1}, \cdots, n_{m}}\left(\prod_{i=1}^{m} x_{i}^{j_{i}} ; x_{1}, \cdots, x_{m}\right)=\prod_{i=1}^{m} B_{n_{i}}\left(x_{i}^{j_{i}} ; x_{i}\right)
\end{array}\right.
$$

(a similar result for one variable can be found in References [1,15] for the first equation of the system (31)). 
3 For $i \in\{1, \cdots, m\}$, let $x_{i} \in[0,1]$ and $\alpha_{i} \in \mathbb{R}$. Then,

$$
B_{n_{1}, \cdots, n_{m}}\left(\exp \left(\sum_{i=1}^{m} \alpha_{i} x_{i}\right) ; x_{1}, \cdots, x_{m}\right)=\prod_{i=1}^{m} B_{n_{i}}\left(\exp \left(\alpha_{i} x_{i}\right) ; x_{i}\right)=\prod_{i=1}^{m}\left[x_{i} \exp \left(\alpha_{i} / n_{i}\right)+\left(1-x_{i}\right)\right]^{n_{i}}
$$

(a similar result for one variable can be found in Reference [1]).

4 For $x_{i} \in[0,1], 1 \leq i \leq m$, we have

$$
\sum_{k_{1}=0}^{n_{1}} \cdots \sum_{k_{m}=0}^{n_{m}} \prod_{i=1}^{m}\left(\begin{array}{l}
n_{i} \\
k_{i}
\end{array}\right)\left(\frac{k_{i}}{n_{i}}-x_{i}\right)^{j_{i}} x_{i}^{k_{i}}\left(1-x_{i}\right)^{n_{i}-k_{i}}=\prod_{i=1}^{m} \sum_{k_{i}=0}^{n_{i}}\left(\begin{array}{l}
n_{i} \\
k_{i}
\end{array}\right)\left(\frac{k_{i}}{n_{i}}-x_{i}\right)^{j_{i}} x_{i}^{k_{i}}\left(1-x_{i}\right)^{n_{i}-k_{i}} .
$$

Proof of Lemma 1. See References [1,15] for the proofs.

\section{Approximation by Bernstein Polynomials with Several Variables}

We express in this theorem our multivariate Bernstein polynomials in terms of differences. A similar result exists for one variable (see References [1,15]).

Theorem 1. Let $f\left(x_{1}, \cdots, x_{m}\right)$ be defined on $[0,1]^{m}$. We have for positive integers $n_{i}, 1 \leq i \leq m$,

$$
B_{n_{1}, \cdots, n_{m}}\left(f ; x_{1}, \cdots, x_{m}\right)=\sum_{t_{1}=0}^{n_{1}} \cdots \sum_{t_{m}=0}^{n_{m}} \triangle_{1}^{t_{1}} \cdots \triangle_{m}^{t_{m}} f_{n_{1}, \cdots, n_{m}}(0, \cdots, 0) \prod_{i=1}^{m}\left(\begin{array}{c}
n_{i} \\
t_{i}
\end{array}\right) x_{i}^{t_{i}}
$$

where $f_{n_{1}, \cdots, n_{m}}\left(t_{1}, \cdots, t_{m}\right)=f\left(\frac{t_{1}}{n_{1}}, \cdots, \frac{t_{m}}{n_{m}}\right)$ and $\triangle_{i}^{t_{i}}, i \in\{1, \cdots, m\}$, is the $t_{i}^{\text {th }}$ difference(s) (see References $[1,15])$ related to the $i^{\text {th }}$ variable $x_{i}$, and applied with step size $h_{i}=\frac{1}{n_{i}}$.

Proof of Theorem 1. We will sometimes use the square brackets to draw attention on computations of certain terms.

$$
\begin{aligned}
& B_{n_{1}, \cdots, n_{m}}\left(f ; x_{1}, \cdots, x_{m}\right) \\
= & \sum_{k_{1}=0}^{n_{1}} \cdots \sum_{k_{m}=0}^{n_{m}} f\left(\frac{k_{1}}{n_{1}}, \cdots, \frac{k_{m}}{n_{m}}\right) \prod_{i=1}^{m}\left(\begin{array}{c}
n_{i} \\
k_{i}
\end{array}\right) x_{i}^{k_{i}}\left(1-x_{i}\right)^{n_{i}-k_{i}} \\
= & \sum_{k_{2}=0}^{n_{2}} \cdots \sum_{k_{m}=0}^{n_{m}}\left[\sum_{k_{1}=0}^{n_{1}} f\left(\frac{k_{1}}{n_{1}}, \frac{k_{2}}{n_{2}}, \cdots, \frac{k_{m}}{n_{m}}\right)\left(\begin{array}{l}
n_{1} \\
k_{1}
\end{array}\right) x_{1}^{k_{1}}\left(1-x_{1}\right)^{n_{1}-k_{1}}\right] \prod_{i=2}^{m}\left(\begin{array}{c}
n_{i} \\
k_{i}
\end{array}\right) x_{i}^{k_{i}}\left(1-x_{i}\right)^{n_{i}-k_{i}} .
\end{aligned}
$$

We take the first variable of $f$ and fix the others. Hence $f\left(\frac{k_{1}}{n_{1}}, \cdots, \frac{k_{m}}{n_{m}}\right)$ is seen as a function $g_{1}\left(\frac{k_{1}}{n_{1}}\right)$, and we apply the following result from P. J. Davis [1], about Bernstein polynomials with one variable:

$$
B_{n}(g ; x)=\sum_{t=0}^{n} \triangle^{t} g_{n}(0)\left(\begin{array}{l}
n \\
t
\end{array}\right) x^{t}
$$

where $n \in \mathbb{N}$ and $g_{n}(t)=g\left(\frac{t}{n}\right)$. Then we get 


$$
\begin{aligned}
& B_{n_{1}, \cdots, n_{m}}\left(f ; x_{1}, \cdots, x_{m}\right) \\
= & \sum_{k_{2}=0}^{n_{2}} \cdots \sum_{k_{m}=0}^{n_{m}}\left(\sum_{t_{1}=0}^{n_{1}} \triangle_{1}^{t_{1}} g_{1}(0) x_{1}^{t_{1}}\left(\begin{array}{c}
n_{1} \\
t_{1}
\end{array}\right)\right) \prod_{i=2}^{m}\left(\begin{array}{c}
n_{i} \\
k_{i}
\end{array}\right) x_{i}^{k_{i}}\left(1-x_{i}\right)^{n_{i}-k_{i},} \\
= & \sum_{k_{2}=0}^{n_{2}} \cdots \sum_{k_{m}=0}^{n_{m}}\left(\sum_{t_{1}=0}^{n_{1}} \triangle_{1}^{t_{1}} f\left(0, \frac{k_{2}}{n_{2}}, \cdots, \frac{k_{m}}{n_{m}}\right) x_{1}^{t_{1}}\left(\begin{array}{c}
n_{1} \\
t_{1}
\end{array}\right)\right) \prod_{i=2}^{m}\left(\begin{array}{c}
n_{i} \\
k_{i}
\end{array}\right) x_{i}^{k_{i}}\left(1-x_{i}\right)^{n_{i}-k_{i}}, \\
= & \sum_{k_{3}=0}^{n_{3}} \cdots \sum_{k_{m}=0}^{n_{m}}\left(\sum_{t_{1}=0}^{n_{1}}\left[\sum_{k_{2}=0}^{n_{2}} \triangle_{1}^{t_{1}} f\left(0, \frac{k_{2}}{n_{2}}, \frac{k_{3}}{n_{3}}, \cdots, \frac{k_{m}}{n_{m}}\right)\left(\begin{array}{c}
n_{2} \\
k_{2}
\end{array}\right) x_{2}^{k_{2}}\left(1-x_{2}\right)^{n_{2}-k_{2}}\right] x_{1}^{t_{1}}\left(\begin{array}{c}
n_{1} \\
t_{1}
\end{array}\right)\right) \\
& \times \prod_{i=3}^{m}\left(\begin{array}{c}
n_{i} \\
k_{i}
\end{array}\right) x_{i}^{k_{i}}\left(1-x_{i}\right)^{n_{i}-k_{i}} .
\end{aligned}
$$

We repeat the action with the second variable of the function $\triangle_{1}^{t_{1}} f\left(0, \frac{k_{2}}{n_{2}}, \cdots, \frac{k_{m}}{n_{m}}\right)$, to get

$$
\begin{aligned}
& B_{n_{1}, \cdots, n_{m}}\left(f ; x_{1}, \cdots, x_{m}\right) \\
= & \sum_{k_{3}=0}^{n_{3}} \cdots \sum_{k_{m}=0}^{n_{m}}\left(\sum_{t_{1}=0}^{n_{1}} \sum_{t_{2}=0}^{n_{2}} \triangle_{1}^{t_{1}} \triangle_{2}^{t_{2}} f\left(0,0, \frac{k_{3}}{n_{3}}, \cdots, \frac{k_{m}}{n_{m}}\right) \prod_{i=1}^{2}\left(\begin{array}{c}
n_{i} \\
t_{i}
\end{array}\right) x_{i}^{t_{i}}\right) \prod_{i=3}^{m}\left(\begin{array}{c}
n_{i} \\
k_{i}
\end{array}\right) x_{i}^{k_{i}}\left(1-x_{i}\right)^{n_{i}-k_{i}} .
\end{aligned}
$$

Then $B_{n_{1}, \cdots, n_{m}}\left(f ; x_{1}, \cdots, x_{m}\right)$

$$
\begin{aligned}
=\sum_{k_{4}=0}^{n_{4}} \cdots \sum_{k_{m}=0}^{n_{m}}\left(\sum_{t_{1}=0}^{n_{1}} \sum_{t_{2}=0}^{n_{2}}\left[\sum_{k_{3}=0}^{n_{3}} \triangle_{1}^{t_{1}} \triangle_{2}^{t_{2}} f\left(0,0, \frac{k_{3}}{n_{3}}, \cdots, \frac{k_{m}}{n_{m}}\right)\left(\begin{array}{l}
n_{3} \\
k_{3}
\end{array}\right) x_{3}^{k_{3}}\left(1-x_{3}\right)^{n_{3}-k_{3}}\right] \prod_{i=1}^{2}\left(\begin{array}{c}
n_{i} \\
t_{i}
\end{array}\right) x_{i}^{t_{i}}\right) \\
\times \prod_{i=4}^{m}\left(\begin{array}{c}
n_{i} \\
k_{i}
\end{array}\right) x_{i}^{k_{i}}\left(1-x_{i}\right)^{n_{i}-k_{i}} .
\end{aligned}
$$

Similarly, with the third variable of $\triangle_{1}^{t_{1}} \triangle_{2}^{t_{2}} f\left(0,0, \frac{k_{3}}{n_{3}}, \cdots, \frac{k_{m}}{n_{m}}\right)$, we get

$$
\begin{aligned}
& B_{n_{1}, \cdots, n_{m}}\left(f ; x_{1}, \cdots, x_{m}\right) \\
= & \sum_{k_{4}=0}^{n_{4}} \cdots \sum_{k_{m}=0}^{n_{m}}\left(\sum_{t_{1}=0}^{n_{1}} \sum_{t_{2}=0}^{n_{2}} \sum_{t_{3}=0}^{n_{3}} \triangle_{1}^{t_{1}} \triangle_{2}^{t_{2}} \triangle_{3}^{t_{3}} f\left(0,0,0, \frac{k_{4}}{n_{4}}, \cdots, \frac{k_{m}}{n_{m}}\right) \prod_{i=1}^{3}\left(\begin{array}{c}
n_{i} \\
t_{i}
\end{array}\right) x_{i}^{t_{i}}\right) \prod_{i=4}^{m}\left(\begin{array}{c}
n_{i} \\
k_{i}
\end{array}\right) x_{i}^{k_{i}}\left(1-x_{i}\right)^{n_{i}-k_{i}} .
\end{aligned}
$$

By repeating, successively, the actions with the $4^{\text {th }}, 5^{\text {th }}, \cdots, m^{\text {th }}$ variables but with the functions $\triangle_{1}^{t_{1}} \triangle_{2}^{t_{2}} \triangle_{3}^{t_{3}} f\left(0,0,0, \frac{k_{4}}{n_{4}}, \cdots, \frac{k_{m}}{n_{m}}\right), \triangle_{1}^{t_{1}} \triangle_{2}^{t_{2}} \triangle_{3}^{t_{3}} \triangle_{4}^{t_{4}} f\left(0,0,0,0, \frac{k_{5}}{n_{5}}, \cdots, \frac{k_{m}}{n_{m}}\right), \cdots, \triangle_{1}^{t_{1}} \triangle_{2}^{t_{2}} \cdots \triangle_{m-1}^{t_{m-1}}$ $f\left(0,0,0, \cdots, 0, \frac{k_{m}}{n_{m}}\right)$ respectively, we obtain the result.

In order to show the pointwise and uniform convergence of our multivariate Bernstein polynomials, let us state and prove the following lemma. A similar result exists for one variable (see References [1,15,21,22]).

Lemma 2. For a given $\delta_{i}>0, i=1, \cdots$, m and $\left(x_{1}, \cdots, x_{m}\right) \in[0,1]^{m}$, we have

$$
\sum_{\left|\frac{k_{1}}{n_{1}}-x_{1}\right| \geq \delta_{1}} \cdots \sum_{\left|\frac{k_{m}}{n_{m}}-x_{m}\right| \geq \delta_{m}} \prod_{i=1}^{m}\left(\begin{array}{c}
n_{i} \\
k_{i}
\end{array}\right) x_{i}^{k_{i}}\left(1-x_{i}\right)^{n_{i}-k_{i}} \leq \frac{1}{4^{m} \prod_{i=1}^{m} n_{i} \delta_{i}^{2}}
$$


summing over those values of $k_{i}=0, \cdots, n_{i}$ for which $\left|\frac{k_{i}}{n_{i}}-x_{i}\right| \geq \delta_{i}$.

Proof of Lemma 2. We will sometimes use the square brackets to draw attention to computations of certain terms.

$$
\begin{aligned}
& \sum_{\left|\frac{k_{1}}{n_{1}}-x_{1}\right| \geq \delta_{1}} \cdots \sum_{\left|\frac{k_{m}}{n_{m}}-x_{m}\right| \geq \delta_{m}} \prod_{i=1}^{m}\left(\begin{array}{c}
n_{i} \\
k_{i}
\end{array}\right) x_{i}^{k_{i}}\left(1-x_{i}\right)^{n_{i}-k_{i}} \\
& =\sum_{\left|\frac{k_{2}}{n_{2}}-x_{2}\right| \geq \delta_{2}} \cdots \sum_{\left|\frac{k_{m}}{n_{m}}-x_{m}\right| \geq \delta_{m}}\left[\sum_{\left|\frac{k_{1}}{n_{1}}-x_{1}\right| \geq \delta_{1}}\left(\begin{array}{l}
n_{1} \\
k_{1}
\end{array}\right) x_{1}^{k_{1}}\left(1-x_{1}\right)^{n_{1}-k_{1}}\right] \prod_{i=2}^{m}\left(\begin{array}{l}
n_{i} \\
k_{i}
\end{array}\right) x_{i}^{k_{i}}\left(1-x_{i}\right)^{n_{i}-k_{i}} \\
& =\left(\sum_{\left|\frac{k_{1}}{n_{1}}-x_{1}\right| \geq \delta_{1}}\left(\begin{array}{l}
n_{1} \\
k_{1}
\end{array}\right) x_{1}^{k_{1}}\left(1-x_{1}\right)^{n_{1}-k_{1}}\right) \sum_{\left|\frac{k_{3}}{n_{3}}-x_{3}\right| \geq \delta_{3}} \cdots \\
& \left.\sum_{\left|\frac{k_{m}}{n_{m}}-x_{m}\right| \geq \delta_{m}} \sum_{\left|\frac{k_{2}}{n_{2}}-x_{2}\right| \geq \delta_{2}}\left(\begin{array}{l}
n_{2} \\
k_{2}
\end{array}\right) x_{2}^{k_{2}}\left(1-x_{2}\right)^{n_{2}-k_{2}}\right] \prod_{i=3}^{m}\left(\begin{array}{l}
n_{i} \\
k_{i}
\end{array}\right) x_{i}^{k_{i}}\left(1-x_{i}\right)^{n_{i}-k_{i}} \\
& =\left(\prod_{i=1}^{2} \sum_{\left|\frac{k_{i}}{n_{i}}-x_{i}\right| \geq \delta_{i}}\left(\begin{array}{c}
n_{i} \\
k_{i}
\end{array}\right) x_{i}^{k_{i}}\left(1-x_{i}\right)^{n_{i}-k_{i}}\right) \underset{\sum_{\left|\frac{k_{3}}{n_{3}}-x_{3}\right| \geq \delta_{3}}}{\cdots} \sum_{\left|\frac{k_{m}}{n_{m}}-x_{m}\right| \geq \delta_{m}} \prod_{i=3}^{m}\left(\begin{array}{c}
n_{i} \\
k_{i}
\end{array}\right) x_{i}^{k_{i}}\left(1-x_{i}\right)^{n_{i}-k_{i}} .
\end{aligned}
$$

By repeating, successively, the actions with $k_{3}, \cdots, k_{m}$, we obtain

$$
\sum_{\left|\frac{k_{1}}{n_{1}}-x_{1}\right| \geq \delta_{1}} \ldots \sum_{\left|\frac{k_{m}}{n_{m}}-x_{m}\right| \geq \delta_{m}} \prod_{i=1}^{m}\left(\begin{array}{c}
n_{i} \\
k_{i}
\end{array}\right) x_{i}^{k_{i}}\left(1-x_{i}\right)^{n_{i}-k_{i}}=\prod_{i=1}^{m} \sum_{\left|\frac{k_{i}}{n_{i}}-x_{i}\right| \geq \delta_{i}}\left(\begin{array}{c}
n_{i} \\
k_{i}
\end{array}\right) x_{i}^{k_{i}}\left(1-x_{i}\right)^{n_{i}-k_{i}}
$$

Let us use the following result which can be found in References [1,21,22]:

$$
\sum_{\left|\frac{k}{n}-x\right| \geq \delta}\left(\begin{array}{l}
n \\
k
\end{array}\right) x^{k}(1-x)^{n-k} \leq \frac{1}{4 n \delta^{2}}
$$

where $n, k \in \mathbb{N}, n \neq 0, k \leq n, \delta>0$ and $x \in[0,1]$. We then get 


$$
\sum_{\left|\frac{k_{1}}{n_{1}}-x_{1}\right| \geq \delta_{1}} \ldots \sum_{\left|\frac{k_{m}}{n_{m}}-x_{m}\right| \geq \delta_{m}} \prod_{i=1}^{m}\left(\begin{array}{c}
n_{i} \\
k_{i}
\end{array}\right) x_{i}^{k_{i}}\left(1-x_{i}\right)^{n_{i}-k_{i}} \leq \prod_{i=1}^{m}\left(\frac{1}{4 n_{i} \delta_{i}^{2}}\right) \leq \frac{1}{4^{m} \prod_{i=1}^{m} n_{i} \delta_{i}^{2}}
$$

Remark 1. Lemma 3 is still valid if the summation is not over all the $k_{i}, 1 \leq i \leq m$ but over some of them. In this case, the upper bound will contain only terms involved in the summation.

The following theorem states for the pointwise and uniform convergence of our Bernstein polynomials with several variables. It should be mentionned that similar work has been done but only for two variables (see (Theorem 1.1 in Reference [26]), [30], (Theorems 3, 5 and 8 in [21]), (Theorem 6.7 in [33]), (Lemma 1 in [28]), (Theorem in [32]) and (Theorems 1 and 3 in [7]).

Theorem 2. Let $f\left(x_{1}, \cdots, x_{m}\right)$ be bounded in the m-dimensional cube $0 \leq x_{i} \leq 1, i=1, \cdots, m$. Then $B_{n_{1}, \cdots, n_{m}}\left(f ; x_{1}, \cdots, x_{m}\right)$ converges towards $f\left(x_{1}, \cdots, x_{m}\right)$ at any point of continuity of this function, as $n_{i} \rightarrow \infty$ for all $i$. If $f \in \mathcal{C}[0,1]^{m}$, the limit holds uniformly in $[0,1]^{m}$.

Proof of Theorem 2. The function $f\left(x_{1}, \cdots, x_{m}\right)$ is assumed bounded in $[0,1]^{m}$. Hence for some $M>0,\left|f\left(x_{1}, \cdots, x_{m}\right)\right| \leq M, \forall\left(x_{1}, \cdots, x_{m}\right) \in[0,1]^{m}$, and therefore for any two points $\left(\alpha_{1}, \cdots, \alpha_{m}\right)$, $\left(\beta_{1}, \cdots, \beta_{m}\right) \in[0,1]^{m}$

$$
\left|f\left(\alpha_{1}, \cdots, \alpha_{m}\right)-f\left(\beta_{1}, \cdots, \beta_{m}\right)\right| \leq 2 M
$$

Let $\left(x_{1}, \cdots, x_{m}\right) \in[0,1]^{m}$ be a point of continuity of $f$. Given $\varepsilon>0$, we can find $\delta_{1}, \cdots, \delta_{m}$ depending on $\left(x_{1}, \cdots, x_{m}\right)$ and $\varepsilon$ such that $\left|f\left(x_{1}, \cdots, x_{m}\right)-f\left(y_{1}, \cdots, y_{m}\right)\right|<\frac{\varepsilon}{2}$, whenever $\left|x_{i}-y_{i}\right|<\delta_{i}, i=1, \cdots, m$.

Since $B_{n_{1}, \cdots, n_{m}}\left(1 ; x_{1}, \cdots, x_{m}\right)=1$, we have

$$
\begin{aligned}
f\left(x_{1}, \cdots, x_{m}\right) & =f\left(x_{1}, \cdots, x_{m}\right) B_{n_{1}, \cdots, n_{m}}\left(1 ; x_{1}, \cdots, x_{m}\right) \\
& =f\left(x_{1}, \cdots, x_{m}\right) \sum_{k_{1}=0}^{n_{1}} \cdots \sum_{k_{m}=0}^{n_{m}} \prod_{i=1}^{m}\left(\begin{array}{l}
n_{i} \\
k_{i}
\end{array}\right) x_{i}^{k_{i}}\left(1-x_{i}\right)^{n_{i}-k_{i}} \\
& =\sum_{k_{1}=0}^{n_{1}} \cdots \sum_{k_{m}=0}^{n_{m}} f\left(x_{1}, \cdots, x_{m}\right) \prod_{i=1}^{m}\left(\begin{array}{l}
n_{i} \\
k_{i}
\end{array}\right) x_{i}^{k_{i}}\left(1-x_{i}\right)^{n_{i}-k_{i}} .
\end{aligned}
$$

Let us consider the set $E=\prod_{i=1}^{m}\left\{0, \cdots, n_{i}\right\}$, and for $j=1, \cdots, m$, we define the sets

$$
\Omega_{j}=\left\{k_{j} \in\left\{0, \cdots, n_{j}\right\}:\left|\frac{k_{j}}{n_{j}}-x_{j}\right|<\delta_{j}\right\} \text { and } F=E \backslash\left(\Omega_{1} \times \cdots \times \Omega_{m}\right)
$$

Then, $F=\bigcup_{k=1}^{m} F_{k}$, with $F_{k}=\left\{\prod_{i=1}^{m} \Omega_{i k}^{\left[\alpha_{i k}\right]} \in F: \alpha_{i k} \in\{0,1\}, \quad \sum_{i=1}^{m} \alpha_{i k}=k\right\}$, where $\Omega_{i k}^{\left[\alpha_{i k}\right]}=\left\{\begin{array}{ll}\Omega_{i} & \text { if } \alpha_{i k}=0 \\ \Omega_{i}^{c} & \text { if } \alpha_{i k}=1\end{array}\right.$ and $\Omega_{i}^{c}=\left\{k_{i} \in\left\{0, \cdots, n_{i}\right\}:\left|\frac{k_{i}}{n_{i}}-x_{i}\right| \geq \delta_{i}\right\}$.

For $A_{k}=\prod_{i=1}^{m} \Omega_{i k}^{\left[\alpha_{i k}\right]} \in F_{k}, k=1, \cdots, m$, let us define also $I_{A_{k}}=\left\{i \in\{1, \cdots, m\}: \alpha_{i k}=1\right\}$ (that means $\left.\operatorname{card}\left(I_{A_{k}}\right)=k \geq 1\right)$. We have 


$$
\begin{aligned}
& \left|f\left(x_{1}, \cdots, x_{m}\right)-B_{n_{1}, \cdots, n_{m}}\left(f ; x_{1}, \cdots, x_{m}\right)\right| \\
= & \mid \sum_{k_{1}=0}^{n_{1}} \cdots \sum_{k_{m}=0}^{n_{m}} f\left(x_{1}, \cdots, x_{m}\right) \prod_{i=1}^{m}\left(\begin{array}{c}
n_{i} \\
k_{i}
\end{array}\right) x_{i}^{k_{i}}\left(1-x_{i}\right)^{n_{i}-k_{i}} \\
& -\sum_{k_{1}=0}^{n_{1}} \cdots \sum_{k_{m}=0}^{n_{m}} f\left(\frac{k_{1}}{n_{1}}, \cdots, \frac{k_{m}}{n_{m}}\right) \prod_{i=1}^{m}\left(\begin{array}{c}
n_{i} \\
k_{i}
\end{array}\right) x_{i}^{k_{i}}\left(1-x_{i}\right)^{n_{i}-k_{i}} \mid, \\
= & \left|\sum_{k_{1}=0}^{n_{1}} \cdots \sum_{k_{m}=0}^{n_{m}}\left[f\left(x_{1}, \cdots, x_{m}\right)-f\left(\frac{k_{1}}{n_{1}}, \cdots, \frac{k_{m}}{n_{m}}\right)\right] \prod_{i=1}^{m}\left(\begin{array}{l}
n_{i} \\
k_{i}
\end{array}\right) x_{i}^{k_{i}}\left(1-x_{i}\right)^{n_{i}-k_{i}}\right|, \\
\leq & \sum_{k_{1}=0}^{n_{1}} \cdots \sum_{k_{m}=0}^{n_{m}}\left|f\left(x_{1}, \cdots, x_{m}\right)-f\left(\frac{k_{1}}{n_{1}}, \cdots, \frac{k_{m}}{n_{m}}\right)\right| \prod_{i=1}^{m}\left(\begin{array}{l}
n_{i} \\
k_{i}
\end{array}\right) x_{i}^{k_{i}}\left(1-x_{i}\right)^{n_{i}-k_{i},}, \\
\leq & \sum_{\Omega_{1}} \cdots \sum_{\Omega_{m}}\left|f\left(x_{1}, \cdots, x_{m}\right)-f\left(\frac{k_{1}}{n_{1}}, \cdots, \frac{k_{m}}{n_{m}}\right)\right| \prod_{i=1}^{m}\left(\begin{array}{c}
n_{i} \\
k_{i}
\end{array}\right) x_{i}^{k_{i}}\left(1-x_{i}\right)^{n_{i}-k_{i}} \\
& +\sum_{F}\left|f\left(x_{1}, \cdots, x_{m}\right)-f\left(\frac{k_{1}}{n_{1}}, \cdots, \frac{k_{m}}{n_{m}}\right)\right| \prod_{i=1}^{m}\left(\begin{array}{c}
n_{i} \\
k_{i}
\end{array}\right) x_{i}^{k_{i}}\left(1-x_{i}\right)^{n_{i}-k_{i} .} .
\end{aligned}
$$

Using the fact that $f$ is continuous and bounded, we get

$$
\begin{aligned}
& \left|f\left(x_{1}, \cdots, x_{m}\right)-B_{n_{1}, \cdots, n_{m}}\left(f ; x_{1}, \cdots, x_{m}\right)\right| \\
\leq & \frac{\varepsilon}{2} \sum_{\Omega_{1}} \cdots \sum_{\Omega_{m}} \prod_{i=1}^{m}\left(\begin{array}{c}
n_{i} \\
k_{i}
\end{array}\right) x_{i}^{k_{i}}\left(1-x_{i}\right)^{n_{i}-k_{i}}+2 M \sum_{F} \prod_{i=1}^{m}\left(\begin{array}{c}
n_{i} \\
k_{i}
\end{array}\right) x_{i}^{k_{i}}\left(1-x_{i}\right)^{n_{i}-k_{i},} \\
\leq & \left.\frac{\varepsilon}{2} \sum_{k_{1}=0}^{n_{1}} \cdots \sum_{k_{m}=0}^{n_{m}} \prod_{i=1}^{m}\left(\begin{array}{c}
n_{i} \\
k_{i}
\end{array}\right) x_{i}^{k_{i}}\left(1-x_{i}\right)^{n_{i}-k_{i}}+2 M \sum_{l=1}^{m} \sum_{A_{l} \in F_{l}} \prod_{i=1}^{m}\left(\begin{array}{c}
n_{i} \\
k_{i}
\end{array}\right) x_{i}^{k_{i}}\left(1-x_{i}\right)^{n_{i}-k_{i}}, \quad \text { since } F=\bigcup_{l=1}^{m} F_{l}\right) \\
\leq & \frac{\varepsilon}{2} B_{n_{1}, \cdots, n_{m}}\left(1 ; x_{1}, \cdots, x_{m}\right)+2 M \sum_{l=1}^{m} \sum_{A_{l} \in F_{l}} \prod_{i=1}^{m}\left(\begin{array}{c}
n_{i} \\
k_{i}
\end{array}\right) x_{i}^{k_{i}}\left(1-x_{i}\right)^{n_{i}-k_{i},} \\
\leq & \frac{\varepsilon}{2}+2 M \sum_{l=1}^{m} \sum_{A_{l} \in F_{l}} \prod_{i=1}^{m}\left(\begin{array}{c}
n_{i} \\
k_{i}
\end{array}\right) x_{i}^{k_{i}}\left(1-x_{i}\right)^{n_{i}-k_{i}} \quad \text { (using Equation (31)). }
\end{aligned}
$$

For $l \in\{1, \cdots, m\}$ and $A_{l} \in F_{l}$,

$$
\sum_{A_{l}} \prod_{i=1}^{m}\left(\begin{array}{c}
n_{i} \\
k_{i}
\end{array}\right) x_{i}^{k_{i}}\left(1-x_{i}\right)^{n_{i}-k_{i}}=\sum_{\Omega_{11}^{\left[\alpha_{n}\right]}} \cdots \sum_{\Omega_{m l}^{\left[a_{m l}\right]}} \prod_{i=1}^{m}\left(\begin{array}{c}
n_{i} \\
k_{i}
\end{array}\right) x_{i}^{k_{i}}\left(1-x_{i}\right)^{n_{i}-k_{i}}=\prod_{i=1}^{m} \sum_{\Omega_{i l}^{\left[a_{i l}\right]}}\left(\begin{array}{c}
n_{i} \\
k_{i}
\end{array}\right) x_{i}^{k_{i}}\left(1-x_{i}\right)^{n_{i}-k_{i}}
$$

using a similar proof as in Lemma 1. For $i \in\{1, \cdots, m\}$,

- $\quad$ if $\alpha_{i l}=0$, this means $i \notin I_{A_{l}}$ then,

$$
\sum_{\Omega_{i l}^{\left[\alpha_{i}\right]}}\left(\begin{array}{c}
n_{i} \\
k_{i}
\end{array}\right) x_{i}^{k_{i}}\left(1-x_{i}\right)^{n_{i}-k_{i}}=\sum_{\Omega_{i}}\left(\begin{array}{c}
n_{i} \\
k_{i}
\end{array}\right) x_{i}^{k_{i}}\left(1-x_{i}\right)^{n_{i}-k_{i}} \leq \sum_{k_{i}=0}^{n_{i}}\left(\begin{array}{c}
n_{i} \\
k_{i}
\end{array}\right) x_{i}^{k_{i}}\left(1-x_{i}\right)^{n_{i}-k_{i}}=1
$$

- $\quad$ if $\alpha_{i l}=1$ that means $i \in I_{A_{l}}$ then

$$
\sum_{\Omega_{i l}^{\left[\alpha_{i}\right]}}\left(\begin{array}{c}
n_{i} \\
k_{i}
\end{array}\right) x_{i}^{k_{i}}\left(1-x_{i}\right)^{n_{i}-k_{i}}=\sum_{\Omega_{i}^{c}}\left(\begin{array}{c}
n_{i} \\
k_{i}
\end{array}\right) x_{i}^{k_{i}}\left(1-x_{i}\right)^{n_{i}-k_{i}} \leq \frac{1}{4 n_{i} \delta_{i}^{2}}
$$


(using Equation (45)).

Hence, for $i, l \in\{1, \cdots, m\}$,

$$
\sum_{\Omega_{i l}^{\left[\alpha_{i l}\right]}}\left(\begin{array}{l}
n_{i} \\
k_{i}
\end{array}\right) x_{i}^{k_{i}}\left(1-x_{i}\right)^{n_{i}-k_{i}} \leq \begin{cases}1 & \text { if } i \notin I_{A_{l}} \\
\frac{1}{4 n_{i} \delta_{i}^{2}} & \text { if } i \in I_{A_{l}}\end{cases}
$$

and this implies

$$
\sum_{A_{l}} \prod_{i=1}^{m}\left(\begin{array}{c}
n_{i} \\
k_{i}
\end{array}\right) x_{i}^{k_{i}}\left(1-x_{i}\right)^{n_{i}-k_{i}} \leq \prod_{i \in I_{A_{l}}} \frac{1}{4 n_{i} \delta_{i}^{2}} .
$$

Therefore,

$$
\begin{aligned}
& \left|f\left(x_{1}, \cdots, x_{m}\right)-B_{n_{1}, \cdots, n_{m}}\left(f ; x_{1}, \cdots, x_{m}\right)\right| \\
\leq & \frac{\varepsilon}{2}+2 M \sum_{l=1}^{m} \sum_{A_{l} \in F_{l}} \prod_{i \in I_{A_{l}}} \frac{1}{4 n_{i} \delta_{i}^{2}}=\frac{\varepsilon}{2}+\sum_{l=1}^{m} \sum_{A_{l} \in F_{l}} \frac{2 M}{\prod_{i \in I_{A_{l}}} 4 n_{i} \delta_{i}^{2}}, \\
\leq & \left.\frac{\varepsilon}{2}+\sum_{l=1}^{m} \sum_{A_{l} \in F_{l}} \frac{2 M}{n^{l} \prod_{i \in I_{A_{l}}} 4 \delta_{i}^{2}} \quad \text { (because card }\left(I_{A_{l}}\right)=l\right), \\
\leq & \frac{\varepsilon}{2}+\frac{1}{n} \sum_{l=1}^{m} \sum_{A_{l} \in F_{l}} \frac{2 M}{\prod_{i \in I_{l}} 4 \delta_{i}^{2}} \quad\left(\text { with } n=\min \left(n_{i}: i=1, \cdots, m\right)\right) .
\end{aligned}
$$

For $n_{1}, \cdots, n_{m}$ sufficiently large (that means $n$ sufficiently large), we have $\frac{1}{n} \sum_{l=1}^{m} \sum_{A_{l} \in F_{l}} \frac{2 M}{\prod_{i \in I_{l}} \delta_{i}^{2}}<\frac{\varepsilon}{2}$. Therefore,

$$
\left|f\left(x_{1}, \cdots, x_{m}\right)-B_{n_{1}, \cdots, n_{m}}\left(f ; x_{1}, \cdots, x_{m}\right)\right| \leq \frac{\varepsilon}{2}+\frac{\varepsilon}{2}=\varepsilon .
$$

Here, it should be noticed that the minimum integer $\tilde{n}$, such that

$$
\left|f\left(x_{1}, \cdots, x_{m}\right)-B_{n_{1}, \cdots, n_{m}}\left(f ; x_{1}, \cdots, x_{m}\right)\right| \leq \varepsilon \quad \forall n_{1}, \cdots, n_{m} \geq n \geq \tilde{n},
$$

depends on $\delta_{1}, \cdots, \delta_{m}$ which themselves depend on $x_{1}, \cdots, x_{m}$ and $\varepsilon$. That is why we only have a pointwise convergence.

Suppose now that $f \in \mathcal{C}[0,1]^{m}$, then $f$ is uniformly continuous on $[0,1]^{m}$ that is, given $\varepsilon>0$, we can find $\delta_{1}, \cdots, \delta_{m}$ depending just on $\varepsilon$, but not on any element in $[0,1]$, such that $\left|f\left(x_{1}, \cdots, x_{m}\right)-f\left(y_{1}, \cdots, y_{m}\right)\right|<\frac{\varepsilon}{2}$, for all $\left(x_{1}, \cdots, x_{m}\right),\left(y_{1}, \cdots, y_{m}\right) \in[0,1]^{m}$ satisfying $\left|x_{i}-y_{i}\right|<\delta_{i}$, $i=1, \cdots, m$. By following the same process, we obtain that

$$
\left|f\left(x_{1}, \cdots, x_{m}\right)-B_{n_{1}, \cdots, n_{m}}\left(f ; x_{1}, \cdots, x_{m}\right)\right| \leq \frac{\varepsilon}{2}+\frac{1}{n} \sum_{l=1}^{m} \frac{2 M}{\prod_{i \in I_{l}} \delta_{i}^{2}}
$$

for all $x_{1}, \cdots, x_{m} \in[0,1]$ and $n=\min \left(n_{i}: i=1, \cdots, m\right)$. Hence, there exist $\tilde{n}$ depending only on $\varepsilon$ and not on $x_{1}, \cdots, x_{m}$ such that

$$
\left|f\left(x_{1}, \cdots, x_{m}\right)-B_{n_{1}, \cdots, n_{m}}\left(f ; x_{1}, \cdots, x_{m}\right)\right|<\varepsilon, \quad \forall x_{1}, \cdots, x_{m} \in[0,1]
$$

and $n_{1}, \cdots, n_{m} \geq n \geq \tilde{n}$. Therefore, the convergence is uniform in $[0,1]^{m}$. 
We express now, in Lemma 3, the $\left(p_{1}, \cdots, p_{m}\right)$-th derivative of our multivariate Bernstein polynomials of order $\left(n_{1}+p_{1}, \cdots, n_{m}+p_{m}\right)$-th in terms of $\left(p_{1}, \cdots, p_{m}\right)$-th differences of the corresponding functions. A similar result exists for one variable (see References [1,15]).

Lemma 3. For $p_{1}, \cdots, p_{m} \geq 0$ integers with $0 \leq p_{i} \leq n_{i}, i=1,2, \cdots, m$, the $\left(p_{1}, \cdots, p_{m}\right)$-th derivative of $B_{n_{1}+p_{1}, \cdots, n_{m}+p_{m}}\left(f ; x_{1}, \cdots, x_{m}\right)$ may be expressed in terms of $\left(p_{1}, \cdots, p_{m}\right)$-th differences of $f$ as

$$
\begin{aligned}
& B_{n_{1}+p_{1}, \cdots, n_{m}+p_{m}}^{p_{1}, \cdots ; x_{1}}\left(f ; \cdots, x_{m}\right) \\
= & {\left[\prod_{i=1}^{m} \frac{\left(n_{i}+p_{i}\right) !}{n_{i} !}\right] \sum_{t_{1}=0}^{n_{1}} \cdots \sum_{t_{m}=0}^{n_{m}} \triangle_{1}^{p_{1}} \cdots \triangle_{m}^{p_{m}} f\left(\frac{t_{1}}{n_{1}+p_{1}}, \cdots, \frac{t_{m}}{n_{m}+p_{m}}\right) \prod_{i=1}^{m}\left(\begin{array}{c}
n_{i} \\
t_{i}
\end{array}\right) x_{i}^{t_{i}}\left(1-x_{i}\right)^{n_{i}-t_{i}} . }
\end{aligned}
$$

Proof of Lemma 3. We will sometimes use the square brackets to draw attention on computations of certain terms. We write

$$
\begin{aligned}
& B_{n_{1}+p_{1}, \cdots, n_{m}+p_{m}}\left(f ; x_{1}, \cdots, x_{m}\right) \\
= & \sum_{k_{1}=0}^{n_{1}+p_{1}} \cdots \sum_{k_{m}=0}^{n_{m}+p_{m}} f\left(\frac{k_{1}}{n_{1}+p_{1}}, \cdots, \frac{k_{m}}{n_{m}+p_{m}}\right) \prod_{i=1}^{m}\left(\begin{array}{l}
n_{i} \\
k_{i}
\end{array}\right) x_{i}^{k_{i}}\left(1-x_{i}\right)^{n_{i}-k_{i}}, \\
= & \sum_{k_{2}=0}^{n_{2}+p_{2}} \cdots \sum_{k_{m}=0}^{n_{m}+p_{m}}\left[\sum_{k_{1}=0}^{n_{1}+p_{1}} f\left(\frac{k_{1}}{n_{1}+p_{1}}, \cdots, \frac{k_{m}}{n_{m}+p_{m}}\right)\left(\begin{array}{l}
n_{1} \\
k_{1}
\end{array}\right) x_{1}^{k_{1}}\left(1-x_{1}\right)^{n_{1}-k_{1}}\right] \\
\times & \prod_{i=2}^{m}\left(\begin{array}{c}
n_{i} \\
k_{i}
\end{array}\right) x_{i}^{k_{i}}\left(1-x_{i}\right)^{n_{i}-k_{i}} .
\end{aligned}
$$

We take the first variable of $f$ and fix the others. Hence, $f\left(\frac{k_{1}}{n_{1}+p_{1}}, \cdots, \frac{k_{m}}{n_{m}+p_{m}}\right)$ is seen as a function $g_{1}\left(\frac{k_{1}}{n_{1}+p_{1}}\right)$ :

$$
\begin{aligned}
& B_{n_{1}+p_{1}, \cdots, n_{m}+p_{m}}\left(f ; x_{1}, \cdots, x_{m}\right) \\
= & \sum_{k_{2}=0}^{n_{2}+p_{2}} \cdots \sum_{k_{m}=0}^{n_{m}+p_{m}}\left[\sum_{k_{1}=0}^{n_{1}+p_{1}} g_{1}\left(\frac{k_{1}}{n_{1}+p_{1}}\right)\left(\begin{array}{l}
n_{1} \\
k_{1}
\end{array}\right) x_{1}^{k_{1}}\left(1-x_{1}\right)^{n_{1}-k_{1}}\right] \prod_{i=2}^{m}\left(\begin{array}{l}
n_{i} \\
k_{i}
\end{array}\right) x_{i}^{k_{i}}\left(1-x_{i}\right)^{n_{i}-k_{i}}, \\
= & \sum_{k_{2}=0}^{n_{2}+p_{2}} \cdots \sum_{k_{m}=0}^{n_{m}+p_{m}} B_{n_{1}+p_{1}}\left(g_{1}, x_{1}\right) \prod_{i=2}^{m}\left(\begin{array}{l}
n_{i} \\
k_{i}
\end{array}\right) x_{i}^{k_{i}}\left(1-x_{i}\right)^{n_{i}-k_{i}} .
\end{aligned}
$$

Hence

$$
B_{n_{1}+p_{1}, \cdots, n_{m}+p_{m}}^{p_{1}}\left(f ; x_{1}, \cdots, x_{m}\right)=\sum_{k_{2}=0}^{n_{2}+p_{2}} \cdots \sum_{k_{m}=0}^{n_{m}+p_{m}} B_{n_{1}+p_{1}}^{p_{1}}\left(g_{1}, x_{1}\right) \prod_{i=2}^{m}\left(\begin{array}{l}
n_{i} \\
k_{i}
\end{array}\right) x_{i}^{k_{i}}\left(1-x_{i}\right)^{n_{i}-k_{i}} .
$$

Let us use the following result from P. J. Davis [1]:

$$
B_{n+p}^{(p)}(f ; x)=\frac{(n+p) !}{n !} \sum_{t=0}^{n} \triangle^{p} f_{n+p}(t)\left(\begin{array}{l}
n \\
t
\end{array}\right) x^{t}(1-x)^{n-t},
$$

where $n, p \in \mathbb{N}$ and $f_{n+p}(t)=f\left(\frac{t}{n+p}\right)$. We then get 


$$
\begin{aligned}
& B_{n_{1}+p_{1}, \cdots, n_{m}+p_{m}}^{p_{1}}\left(f ; x_{1}, \cdots, x_{m}\right) \\
& =\sum_{k_{2}=0}^{n_{2}+p_{2}} \cdots \sum_{k_{m}=0}^{n_{m}+p_{m}}\left(\frac{\left(n_{1}+p_{1}\right) !}{n !} \sum_{t_{1}=0}^{n_{1}} \triangle_{1}^{p_{1}} g_{1}\left(\frac{t_{1}}{n_{1}+p_{1}}\right)\left(\begin{array}{c}
n_{1} \\
t_{1}
\end{array}\right) x_{1}^{t_{1}}\left(1-x_{1}\right)^{n_{1}-t_{1}}\right) \prod_{i=2}^{m}\left(\begin{array}{c}
n_{i} \\
k_{i}
\end{array}\right) x_{i}^{k_{i}}\left(1-x_{i}\right)^{n_{i}-k_{i}}, \\
& =\sum_{k_{2}=0}^{n_{2}+p_{2}} \cdots \sum_{k_{m}=0}^{n_{m}+p_{m}}\left(\frac{\left(n_{1}+p_{1}\right) !}{n !} \sum_{t_{1}=0}^{n_{1}} \triangle_{1}^{p_{1}} f\left(\frac{t_{1}}{n_{1}+p_{1}}, \frac{k_{2}}{n_{2}+p_{2}}, \cdots, \frac{k_{m}}{n_{m}+p_{m}}\right)\left(\begin{array}{c}
n_{1} \\
t_{1}
\end{array}\right) x_{1}^{t_{1}}\left(1-x_{1}\right)^{n_{1}-t_{1}}\right) \\
& \times \prod_{i=2}^{m}\left(\begin{array}{c}
n_{i} \\
k_{i}
\end{array}\right) x_{i}^{k_{i}}\left(1-x_{i}\right)^{n_{i}-k_{i}}, \\
& =\sum_{k_{3}=0}^{n_{3}+p_{3}} \cdots \sum_{k_{m}=0}^{n_{m}+p_{m}}\left(\frac { ( n _ { 1 } + p _ { 1 } ) ! } { n ! } \sum _ { t _ { 1 } = 0 } ^ { n _ { 1 } } \left[\sum_{k_{2}=0}^{n_{2}+p_{2}} \triangle_{1}^{p_{1}} f\left(\frac{t_{1}}{n_{1}+p_{1}}, \frac{k_{2}}{n_{2}+p_{2}}, \cdots, \frac{k_{m}}{n_{m}+p_{m}}\right)\right.\right. \\
& \left.\left.\times\left(\begin{array}{l}
n_{2} \\
k_{2}
\end{array}\right) x_{2}^{k_{2}}\left(1-x_{2}\right)^{n_{2}-k_{2}}\right]\left(\begin{array}{l}
n_{1} \\
t_{1}
\end{array}\right) x_{1}^{t_{1}}\left(1-x_{1}\right)^{n_{1}-t_{1}}\right) \prod_{i=3}^{m}\left(\begin{array}{c}
n_{i} \\
k_{i}
\end{array}\right) x_{i}^{k_{i}}\left(1-x_{i}\right)^{n_{i}-k_{i}} .
\end{aligned}
$$
we get

We repeat the action with the second variable of the function $\triangle_{1}^{p_{1}} f\left(\frac{t_{1}}{n_{1}+p_{1}}, \frac{k_{2}}{n_{2}+p_{2}}, \cdots, \frac{k_{m}}{n_{m}+p_{m}}\right)$,

$$
\begin{aligned}
& B_{n_{1}+p_{1}, \cdots, n_{m}+p_{m}}^{p_{1}, p_{2}}\left(f ; x_{1}, \cdots, x_{m}\right) \\
= & \sum_{k_{3}=0}^{n_{3}+p_{3}} \cdots \sum_{k_{m}=0}^{n_{m}+p_{m}}\left(( \prod _ { i = 1 } ^ { 2 } \frac { ( n _ { i } + p _ { i } ) ! } { n _ { i } ! } ) \sum _ { t _ { 1 } = 0 } ^ { n _ { 1 } } \sum _ { t _ { 2 } = 0 } ^ { n _ { 2 } } \triangle _ { 1 } ^ { p _ { 1 } } \triangle _ { 2 } ^ { p _ { 2 } } f \left(\frac{t_{1}}{n_{1}+p_{1}}, \frac{t_{2}}{n_{2}+p_{2}},\right.\right. \\
& \left.\left.\frac{k_{3}}{n_{3}+p_{3}}, \cdots, \frac{k_{m}}{n_{m}+p_{m}}\right) \prod_{i=1}^{2}\left(\begin{array}{c}
n_{i} \\
t_{i}
\end{array}\right) x_{i}^{t_{i}}\left(1-x_{i}\right)^{n_{i}-t_{i}}\right) \prod_{i=3}^{m}\left(\begin{array}{c}
n_{i} \\
k_{i}
\end{array}\right) x_{i}^{k_{i}}\left(1-x_{i}\right)^{n_{i}-k_{i}}, \\
= & \sum_{k_{3}=0}^{n_{3}+p_{3}} \cdots \sum_{k_{m}=0}^{n_{m}+p_{m}}\left(( \prod _ { i = 1 } ^ { 2 } \frac { ( n _ { i } + p _ { i } ) ! } { n _ { i } ! } ) \sum _ { t _ { 1 } = 0 } ^ { n _ { 1 } } \sum _ { t _ { 2 } = 0 } ^ { n _ { 2 } } \sum _ { n _ { 3 } = 0 } ^ { n _ { 3 } + p _ { 3 } } \sum _ { k _ { 3 } = 0 } ^ { p _ { 1 } } \triangle _ { 2 } ^ { p _ { 2 } } f \left(\frac{t_{1}}{n_{1}+p_{1}}, \frac{t_{2}}{n_{2}+p_{2}},\right.\right. \\
& \left.\left.\left.\frac{k_{3}}{n_{3}+p_{3}}, \cdots, \frac{k_{m}}{n_{m}+p_{m}}\right)\left(\begin{array}{c}
n_{3} \\
k_{3}
\end{array}\right) x_{3}^{k_{3}}\left(1-x_{3}\right)^{n_{3}-k_{3}}\right] \prod_{i=1}^{2}\left(\begin{array}{c}
n_{i} \\
t_{i}
\end{array}\right) x_{i}^{t_{i}}\left(1-x_{i}\right)^{n_{i}-t_{i}}\right) \prod_{i=4}^{m}\left(\begin{array}{c}
n_{i} \\
k_{i}
\end{array}\right) x_{i}^{k_{i}}\left(1-x_{i}\right)^{n_{i}-k_{i}} .
\end{aligned}
$$

After, we continue the same action with the third variable of $\triangle_{1}^{t_{1}} \triangle_{2}^{t_{2}} f\left(\frac{t_{1}}{n_{1}+p_{1}}, \frac{t_{2}}{n_{2}+p_{2}}, \frac{k_{3}}{n_{3}+p_{3}}, \cdots\right.$, $\left.\frac{k_{m}}{n_{m}+p_{m}}\right)$ and get

$$
\begin{aligned}
& B_{n_{1}+p_{1}, \cdots, n_{m}+p_{m}}^{p_{1}, p_{2}, p_{3}}\left(f ; x_{1}, \cdots, x_{m}\right) \\
= & \sum_{k_{4}=0}^{n_{4}+p_{4}} \cdots \sum_{k_{m}=0}^{n_{m}+p_{m}}\left(( \prod _ { i = 1 } ^ { 3 } \frac { ( n _ { i } + p _ { i } ) ! } { n _ { i } ! } ) \sum _ { t _ { 2 } = 0 } ^ { n _ { 2 } } \triangle _ { 1 } ^ { p _ { 1 } } \triangle _ { 2 } ^ { p _ { 2 } } f \left(\frac{t_{1}}{n_{1}+p_{1}}, \frac{t_{2}}{n_{2}+p_{2}}, \frac{t_{3}}{n_{3}+p_{3}},\right.\right. \\
& \left.\left.\frac{k_{4}}{n_{4}+p_{4}}, \cdots, \frac{k_{m}}{n_{m}+p_{m}}\right) \prod_{i=1}^{3}\left(\begin{array}{c}
n_{i} \\
t_{i}
\end{array}\right) x_{i}^{t_{i}}\left(1-x_{i}\right)^{n_{i}-k_{i}}\right) \prod_{i=4}^{m}\left(\begin{array}{c}
n_{i} \\
k_{i}
\end{array}\right) x_{i}^{k_{i}}\left(1-x_{i}\right)^{n_{i}-k_{i}},
\end{aligned}
$$

which can be written as 


$$
\begin{gathered}
B_{n_{1}+p_{1}, \cdots, n_{m}+p_{m}}^{p_{1}, p_{2}, p_{3}}\left(f ; x_{1}, \cdots, x_{m}\right) \\
=\sum_{k_{5}=0}^{n_{5}+p_{5}} \cdots \sum_{k_{m}=0}^{n_{m}+p_{m}}\left(( \prod _ { i = 1 } ^ { 3 } \frac { ( n _ { i } + p _ { i } ) ! } { n _ { i } ! } ) \sum _ { t _ { 1 } = 0 } ^ { n _ { 1 } } \sum _ { t _ { 2 } = 0 } ^ { n _ { 2 } } \sum _ { t _ { 3 } = 0 } ^ { n _ { 3 } } \left[\sum _ { k _ { 4 } = 0 } ^ { n _ { 4 } + p _ { 4 } } \triangle _ { 1 } ^ { p _ { 1 } } \triangle _ { 2 } ^ { p _ { 2 } } \triangle _ { 3 } ^ { p _ { 3 } } f \left(\frac{t_{1}}{n_{1}+p_{1}}, \frac{t_{2}}{n_{2}+p_{2}},\right.\right.\right. \\
\left.\left.\left.\frac{t_{3}}{n_{3}+p_{3}}, \frac{k_{4}}{n_{4}+p_{4}}, \cdots, \frac{k_{m}}{n_{m}+p_{m}}\right)\left(\begin{array}{c}
n_{4} \\
t_{4}
\end{array}\right) x_{4}^{t_{4}}\left(1-x_{4}\right)^{n_{4}-k_{4}}\right] \prod_{i=1}^{3}\left(\begin{array}{c}
n_{i} \\
t_{i}
\end{array}\right) x_{i}^{t_{i}}\left(1-x_{i}\right)^{n_{i}-k_{i}}\right) \\
\times \prod_{i=5}^{m}\left(\begin{array}{c}
n_{i} \\
k_{i}
\end{array}\right) x_{i}^{k_{i}}\left(1-x_{i}\right)^{n_{i}-k_{i}} .
\end{gathered}
$$

By repeating successively the actions with the $4-$ th, $5-$ th, $\cdots, m-$ th variables but with the functions $\triangle_{1}^{t_{1}} \triangle_{2}^{t_{2}} \triangle_{3}^{t_{3}} f\left(\frac{t_{1}}{n_{1}+p_{1}}, \frac{t_{2}}{n_{2}+p_{2}}, \frac{t_{3}}{n_{3}+p_{3}}, \frac{k_{4}}{n_{4}+p_{4}}, \cdots, \frac{k_{m}}{n_{m}+p_{m}}\right)$, $\triangle_{1}^{t_{1}} \triangle_{2}^{t_{2}} \triangle_{3}^{t_{3}} \triangle_{4}^{t_{4}} f\left(\frac{t_{1}}{n_{1}+p_{1}}, \frac{t_{2}}{n_{2}+p_{2}}, \frac{t_{3}}{n_{3}+p_{3}}, \frac{t_{4}}{n_{4}+p_{4}}, \frac{k_{5}}{n_{5}+p_{5}}, \cdots, \frac{k_{m}}{n_{m}+p_{m}}\right), \cdots$, $\triangle_{1}^{t_{1}} \triangle_{2}^{t_{2}} \cdots \triangle_{m-1}^{t_{m-1}} f\left(\frac{t_{1}}{n_{1}+p_{1}}, \frac{t_{2}}{n_{2}+p_{2}}, \cdots, \frac{t_{m-1}}{n_{m-1}+p_{m-1}}, \frac{k_{m}}{n_{m}+p_{m}}\right)$ respectively, we obtain the result.

Let us see how to deduce the monotonicity, the fixed sign of the $\left(p_{1}, \cdots, p_{m}\right)$-th derivative, the convexity, and the upper and lower bounds of our multivariate Bernstein polynomials from those of the corresponding function. Similar results exist for one variable (see References $[1,21,31]$ ).

Theorem 3. Let $0 \leq p_{i} \leq n_{i}, i=1, \cdots, m$, be fixed integers and the restriction of $f$ to each of its variables $i$ belong to $\mathcal{C}^{p_{i}}[0,1], i=1, \cdots, m$. If

$$
\begin{aligned}
A & \leq f^{\left(p_{1}\right) \cdots\left(p_{m}\right)}\left(x_{1}, \cdots, x_{m}\right) \leq B, \quad\left(x_{1}, \cdots, x_{m}\right) \in[0,1]^{m}, \\
\text { then } A & \leq \prod_{i=1}^{m} \frac{n_{i}^{p_{i}}}{n_{i}\left(n_{i}-1\right) \cdots\left(n_{i}-p_{i}+1\right)} B_{n_{1}, \cdots, n_{m}}^{p_{1}, \cdots, p_{m}}\left(f ; x_{1}, \cdots, x_{m}\right) \leq B
\end{aligned}
$$

with $\left(x_{1}, \cdots, x_{m}\right) \in[0,1]^{m}$. For all $p_{i}=0, i=1, \cdots, m$, the multiplier of $B_{n_{1}, \cdots, n_{m}}^{p_{1}, \cdots, p_{m}}$ is to be interpreted as 1 .

- If $f^{\left(p_{1}\right) \cdots\left(p_{m}\right)}\left(x_{1}, \cdots, x_{m}\right) \geq 0,\left(x_{1}, \cdots, x_{m}\right) \in[0,1]^{m}$, then

$$
B_{n_{1}, \cdots, n_{m}}^{p_{1}, \cdots, p_{m}}\left(f ; x_{1}, \cdots, x_{m}\right) \geq 0,\left(x_{1}, \cdots, x_{m}\right) \in[0,1]^{m}
$$

- If $f\left(x_{1}, \cdots, x_{m}\right)$ is convex on $[0,1]^{m}$, then $B_{n_{1}, \cdots, n_{m}}\left(f ; x_{1}, \cdots, x_{m}\right)$ is convex on $[0,1]^{m}$.

Proof of Theorem 3. We begin with Equation (62) and replace $n_{i}$ by $n_{i}-p_{i}, i=1, \cdots, m$. We have

$$
\begin{gathered}
B_{n_{1}, \cdots, n_{m}}^{p_{1}, \cdots, p_{m}}\left(f ; x_{1}, \cdots, x_{m}\right)=\left(\prod_{i=1}^{m} \frac{n_{i} !}{\left(n_{i}-p_{i}\right) !}\right) \sum_{t_{1}=0}^{n_{1}-p_{1}} \cdots \sum_{t_{m}=0}^{n_{m}-p_{m}} \triangle_{1}^{p_{1}} \cdots \triangle_{m}^{p_{m}} f\left(\frac{t_{1}}{n_{1}}, \cdots, \frac{t_{m}}{n_{m}}\right) \\
\times \prod_{i=1}^{m}\left(\begin{array}{c}
n_{i} \\
t_{i}
\end{array}\right) x_{i}^{t_{i}}\left(1-x_{i}\right)^{n_{i}-p_{i}-t_{i}} .
\end{gathered}
$$

Let us use the following result from P. J. Davis [1]: for $g \in \mathcal{C}^{p}[a, b]$ and $a=e_{0}<e_{1}<\cdots<e_{n}=b$ with $e_{i}=e_{0}+i h \in \mathbb{R}, h=e_{i+1}-e_{i}$, a subdivision of $[a, b]$,

$$
\left.\exists \varepsilon_{0} \in\right] e_{0}, e_{p}\left[, \quad \frac{\triangle^{p} g\left(x_{0}\right)}{h^{p}}=g^{(p)}\left(\varepsilon_{0}\right)\right.
$$


Then we get for any variable of $f$ with step size $h_{i}=\frac{1}{n_{i}}, i=1, \cdots, m$,

$$
\triangle_{1}^{p_{1}} \cdots \triangle_{m}^{p_{m}} f\left(\frac{t_{1}}{n_{1}}, \cdots, \frac{t_{m}}{n_{m}}\right)=\frac{f^{\left(p_{1}\right) \cdots\left(p_{m}\right)}\left(\varepsilon_{t_{1}}, \cdots, \varepsilon_{t_{m}}\right)}{n_{1}^{p_{1}} \cdots n_{m}^{p_{m}}},
$$

where $\frac{t_{i}}{n_{i}}<\varepsilon_{t_{i}}<\frac{t_{i}+p_{i}}{n_{i}}, i=1, \cdots, m$. Thus,

$$
\begin{aligned}
& B_{n_{1}, \cdots, n_{m}}^{p_{1}, \cdots, p_{m}}\left(f ; x_{1}, \cdots, x_{m}\right) \\
& =\left(\prod_{i=1}^{m} \frac{n_{i} !}{\left(n_{i}-p_{i}\right) !}\right) \sum_{t_{1}=0}^{n_{1}-p_{1}} \cdots \sum_{t_{m}=0}^{n_{m}-p_{m}} \frac{f^{\left(p_{1}\right) \cdots\left(p_{m}\right)}\left(\varepsilon_{t_{1}}, \cdots, \varepsilon_{t_{m}}\right)}{n_{1}^{p_{1}} \cdots n_{m}^{p_{m}}} \prod_{i=1}^{m}\left(\begin{array}{c}
n_{i}-p_{i} \\
t_{i}
\end{array}\right) x_{i}^{t_{i}}\left(1-x_{i}\right)^{n_{i}-p_{i}-t_{i}} \\
& =\left(\prod_{i=1}^{m} \frac{n_{i}\left(n_{i}-1\right) \cdots\left(n_{i}-p_{i}+1\right)}{n_{i}^{p_{i}}}\right) \sum_{t_{1}=0}^{n_{1}-p_{1}} \cdots \sum_{t_{m}=0}^{n_{m}-p_{m}} f^{\left(p_{1}\right) \cdots\left(p_{m}\right)}\left(\varepsilon_{t_{1}}, \cdots, \varepsilon_{t_{m}}\right) \\
& \times \prod_{i=1}^{m}\left(\begin{array}{c}
n_{i}-p_{i} \\
t_{i}
\end{array}\right) x_{i}^{t_{i}}\left(1-x_{i}\right)^{n_{i}-p_{i}-t_{i}} \\
& A \leq f^{\left(p_{1}\right) \cdots\left(p_{m}\right)}\left(x_{1}, \cdots, x_{m}\right) \leq B, \quad\left(x_{1}, \cdots, x_{m}\right) \in[0,1]^{m} \\
& \Longrightarrow A \leq f^{\left(p_{1}\right) \cdots\left(p_{m}\right)}\left(\varepsilon_{t_{1}}, \cdots, \varepsilon_{t_{m}}\right) \leq B \\
& \Longrightarrow A \leq f^{\left(p_{1}\right) \cdots\left(p_{m}\right)}\left(\varepsilon_{t_{1}}, \cdots, \varepsilon_{t_{m}}\right) \sum_{t_{1}=0}^{n_{1}-p_{1}} \cdots \sum_{t_{m}=0}^{n_{m}-p_{m}} \prod_{i=1}^{m}\left(\begin{array}{c}
n_{i}-p_{i} \\
t_{i}
\end{array}\right) x_{i}^{t_{i}}\left(1-x_{i}\right)^{n_{i}-p_{i}-t_{i}} \leq B \\
& \text { (since } \left.\sum_{t_{1}=0}^{n_{1}-p_{1}} \cdots \sum_{t_{m}=0}^{n_{m}-p_{m}} \prod_{i=1}^{m}\left(\begin{array}{c}
n_{i}-p_{i} \\
t_{i}
\end{array}\right) x_{i}^{t_{i}}\left(1-x_{i}\right)^{n_{i}-p_{i}-t_{i}}=1\right) \\
& \Longrightarrow A \leq \sum_{t_{1}=0}^{n_{1}-p_{1}} \cdots \sum_{t_{m}=0}^{n_{m}-p_{m}} f^{\left(p_{1}\right) \cdots\left(p_{m}\right)}\left(\varepsilon_{t_{1}}, \cdots, \varepsilon_{t_{m}}\right) \prod_{i=1}^{m}\left(\begin{array}{c}
n_{i}-p_{i} \\
t_{i}
\end{array}\right) x_{i}^{t_{i}}\left(1-x_{i}\right)^{n_{i}-p_{i}-t_{i}} \leq B \\
& \Longrightarrow A \leq\left(\prod_{i=1}^{m} \frac{n_{i}^{p_{i}}}{n_{i}\left(n_{i}-1\right) \cdots\left(n_{i}-p_{i}+1\right)}\right) B_{n_{1}, \cdots, n_{m}}^{p_{1}, \cdots, p_{m}}\left(f ; x_{1}, \cdots, x_{m}\right) \leq B \quad \text { for all } \\
& \left.\left(x_{1}, \cdots, x_{m}\right) \in[0,1]^{m} \text { (using }(77)\right) \text {. }
\end{aligned}
$$

When all $p_{i}=0$, the multiplier of $B_{n_{1}, \cdots, n_{m}}^{p_{1}, \cdots, p_{m}}$ is

$$
\prod_{i=1}^{m} \frac{n_{i}^{p_{i}}}{n_{i}\left(n_{i}-1\right) \cdots\left(n_{i}-p_{i}+1\right)}=\prod_{i=1}^{m} \frac{\left(n_{i}-p_{i}\right) ! n^{p_{i}}}{n_{i} !}=1 \quad\left(p_{i}=0 \forall i=1, \cdots, m\right) .
$$

* If $f^{\left(p_{1}\right) \cdots\left(p_{m}\right)}\left(x_{1}, \cdots, x_{m}\right) \geq 0,\left(x_{1}, \cdots, x_{m}\right) \in[0,1]^{m}$, we set $A=0$ and obtain

$$
0 \leq\left(\prod_{i=1}^{m} \frac{n_{i}^{p_{i}}}{n_{i}\left(n_{i}-1\right) \cdots\left(n_{i}-p_{i}+1\right)}\right) B_{n_{1}, \cdots, n_{m}}^{p_{1}, \cdots, p_{m}}\left(f ; x_{1}, \cdots, x_{m}\right)
$$

for all $\left(x_{1}, \cdots, x_{m}\right) \in[0,1]^{m}$. This means $B_{n_{1}, \cdots, n_{m}}^{p_{1}, \cdots, p_{m}}\left(f ; x_{1}, \cdots, x_{m}\right) \geq 0,\left(x_{1}, \cdots, x_{m}\right) \in[0,1]^{m}$. 
* If $f$ is convex, $\triangle_{1}^{2} \cdots \triangle_{m}^{2} f\left(\frac{t_{1}}{n_{1}}, \cdots, \frac{t_{m}}{n_{m}}\right) \geq 0$ and hence we have $\frac{f^{(2) \cdots(2)}\left(\varepsilon_{t_{1}}, \cdots, \varepsilon_{t_{m}}\right)}{n_{1}^{2} \cdots n_{m}^{2}} \geq 0$ since $\frac{f^{\left(p_{1}\right) \cdots\left(p_{m}\right)}\left(\varepsilon_{t_{1}}, \cdots, \varepsilon_{t_{m}}\right)}{n_{1}^{p_{1}} \cdots n_{m}^{p_{m}}}=\triangle_{1}^{p_{1}} \cdots \triangle_{m}^{p_{m}} f\left(\frac{t_{1}}{n_{1}}, \cdots, \frac{t_{m}}{n_{m}}\right)$, this means $f^{(2) \cdots(2)}\left(\varepsilon_{t_{1}}, \cdots, \varepsilon_{t_{m}}\right) \geq 0$. Thus, from (77) with $p_{i}=2 \forall i=1, \cdots, m$,

$$
B_{n_{1}, \cdots, n_{m}}^{2, \cdots, 2}\left(f ; x_{1}, \cdots, x_{m}\right) \geq 0,\left(x_{1}, \cdots, x_{m}\right) \in[0,1]^{m} .
$$

This implies that $B_{n_{1}, \cdots, n_{m}}$ is convex in every closed interval of $(0,1)^{m}$. Since $B_{n_{1}, \cdots, n_{m}}$ is continuous, it is convex in $[0,1]^{m}$.

Corollary 1. If $f\left(x_{1}, \cdots, x_{m}\right)$ is decreasing (resp. nondecreasing) on $[0,1]$ with respect to the variable $x_{i}, i \in$ $\{1, \cdots, m\}$, then $B_{n_{1}, \cdots, n_{m}}\left(f ; x_{1}, \cdots, x_{m}\right)$ is decreasing (resp. nondecreasing) with respect to the variable $x_{i}$, $i \in\{1, \cdots, m\}$ on $[-1,1]$.

Proof of Corollary 1. We just use Theorem 3, and the rest follows easily.

The following theorem states for the pointwise and uniform convergence of the $\left(p_{1}, \cdots, p_{m}\right)^{\text {th }}$ derivative of our multivariate Bernstein polynomials, compare ([35] Theorem of Kingsley) and ([28] Theorem 1) (just for two variables), and ([25] Theorem 4) and ([7] Theorem 2).

Theorem 4. Let $0 \leq p_{i} \leq n_{i}, i=0, \cdots, m$, be fixed integers, $f$ uniformly continuous on $[0,1]^{m}$ and $\frac{\partial^{p_{i}} f}{\partial x_{i}^{p_{i}}}$ also uniformly continuous on $[0,1]^{m}, i=1, \cdots, m$. Then $B_{n_{1}, \cdots, n_{m}}^{p_{1}, \cdots, p_{m}}\left(f ; x_{1}, \cdots, x_{m}\right)$ converges towards $f^{\left(p_{1}\right) \cdots\left(p_{m}\right)}\left(x_{1}, \cdots, x_{m}\right)$ uniformly on $[0,1]^{m}$.

Proof of Theorem 4. We have already shown that the the above result holds for $p_{i}=0, \forall i=0, \cdots, m$. We have to look at the case where $p_{i}$ are not all zero.

We begin with the expression for $B_{n_{1}+p_{1}, \cdots, n_{m}+p_{m}}^{p_{1}, \cdots, p_{m}}\left(f ; x_{1}, \cdots, x_{m}\right)$ given in Equation (62) and write (using Equation (75) with $h_{i}=\frac{1}{n_{i}+p_{i}}, i=1, \cdots, m$ )

$$
\triangle_{1}^{p_{1}} \cdots \triangle_{m}^{p_{m}} f\left(\frac{t_{1}}{n_{1}+p_{1}}, \cdots, \frac{t_{m}}{n_{m}+p_{m}}\right)=\frac{f^{\left(p_{1}\right) \cdots\left(p_{m}\right)}\left(\varepsilon_{t_{1}}, \cdots, \varepsilon_{t_{m}}\right)}{\prod_{i=1}^{m}\left(n_{i}+p_{i}\right)^{p_{i}}}
$$

where $\frac{t_{i}}{n_{i}+p_{i}}<\varepsilon_{t_{i}}<\frac{t_{i}+p_{i}}{n_{i}+p_{i}}, \quad i=1, \cdots, m$. We get

$$
\begin{aligned}
& B_{n_{1}+p_{1}, \cdots, n_{m}+p_{m}}^{p_{1}, \cdots},\left(f ; x_{1}, \cdots, x_{m}\right) \\
= & \left(\prod_{i=1}^{m} \frac{\left(n_{i}+p_{i}\right) !}{n_{i} !}\right) \sum_{t_{1}=0}^{n_{1}} \cdots \sum_{t_{m}=0}^{n_{m}} \triangle_{1}^{p_{1}} \cdots \triangle_{m}^{p_{m}} f\left(\frac{t_{1}}{n_{1}+p_{1}}, \cdots, \frac{t_{m}}{n_{m}+p_{m}}\right) \prod_{i=1}^{m}\left(\begin{array}{c}
n_{i} \\
t_{i}
\end{array}\right) x_{i}^{t_{i}}\left(1-x_{i}\right)^{n_{i}-t_{i}} \\
= & \left(\prod_{i=1}^{m} \frac{\left(n_{i}+p_{i}\right) !}{n_{i} !\left(n_{i}+p_{i}\right)}\right) \sum_{t_{1}=0}^{n_{1}} \cdots \sum_{t_{m}=0}^{n_{m}} f^{\left(p_{1}\right) \cdots\left(p_{m}\right)}\left(\varepsilon_{t_{1}}, \cdots, \varepsilon_{t_{m}}\right) \prod_{i=1}^{m}\left(\begin{array}{c}
n_{i} \\
t_{i}
\end{array}\right) x_{i}^{t_{i}}\left(1-x_{i}\right)^{n_{i}-t_{i},}
\end{aligned}
$$

which gives us 


$$
\begin{aligned}
& \left(\prod_{i=1}^{m} \frac{n_{i} !\left(n_{i}+p_{i}\right)^{p_{i}}}{\left(n_{i}+p_{i}\right) !}\right) B_{n_{1}+p_{1}, \cdots, n_{m}+p_{m}}^{p_{1}, \cdots, p_{m}}\left(f ; x_{1}, \cdots, x_{m}\right) \\
& \quad=\sum_{t_{1}=0}^{n_{1}} \cdots \sum_{t_{m}=0}^{n_{m}} f^{\left(p_{1}\right) \cdots\left(p_{m}\right)}\left(\varepsilon_{t_{1}}, \cdots, \varepsilon_{t_{m}}\right) \prod_{i=1}^{m}\left(\begin{array}{c}
n_{i} \\
t_{i}
\end{array}\right) x_{i}^{t_{i}}\left(1-x_{i}\right)^{n_{i}-t_{i}} .
\end{aligned}
$$

We then approximate $f^{\left(p_{1}\right) \cdots\left(p_{m}\right)}\left(\varepsilon_{t_{1}}, \cdots, \varepsilon_{t_{m}}\right)$ writing

$$
\begin{aligned}
& f^{\left(p_{1}\right) \cdots\left(p_{m}\right)}\left(\varepsilon_{t_{1}}, \cdots, \varepsilon_{t_{m}}\right) \\
= & \left(f^{\left(p_{1}\right) \cdots\left(p_{m}\right)}\left(\varepsilon_{t_{1}}, \cdots, \varepsilon_{t_{m}}\right)-f^{\left(p_{1}\right) \cdots\left(p_{m}\right)}\left(\frac{t_{1}}{n_{1}}, \cdots, \frac{t_{m}}{n_{m}}\right)\right)+f^{\left(p_{1}\right) \cdots\left(p_{m}\right)}\left(\frac{t_{1}}{n_{1}}, \cdots, \frac{t_{m}}{n_{m}}\right) .
\end{aligned}
$$

We thus obtain

$$
\begin{aligned}
& \left(\prod_{i=1}^{m} \frac{n_{i} !\left(n_{i}+p_{i}\right)^{p_{i}}}{\left(n_{i}+p_{i}\right) !}\right) B_{n_{1}+p_{1}, \cdots, n_{m}+p_{m}}^{p_{1}, \cdots, p_{m}}\left(f ; x_{1}, \cdots, x_{m}\right) \\
& =\sum_{t_{1}=0}^{n_{1}} \cdots \sum_{t_{m}=0}^{n_{m}}\left(f^{\left(p_{1}\right) \cdots\left(p_{m}\right)}\left(\frac{t_{1}}{n_{1}}, \cdots, \frac{t_{m}}{n_{m}}\right)+\left(f^{\left(p_{1}\right) \cdots\left(p_{m}\right)}\left(\varepsilon_{t_{1}}, \cdots, \varepsilon_{t_{m}}\right)\right.\right. \\
& \left.\left.-f^{\left(p_{1}\right) \cdots\left(p_{m}\right)}\left(\frac{t_{1}}{n_{1}}, \cdots, \frac{t_{m}}{n_{m}}\right)\right)\right) \prod_{i=1}^{m}\left(\begin{array}{c}
n_{i} \\
t_{i}
\end{array}\right) x_{i}^{t_{i}}\left(1-x_{i}\right)^{n_{i}-t_{i}} \\
& =T_{1}\left(x_{1}, \cdots, x_{m}\right)+T_{2}\left(x_{1}, \cdots, x_{m}\right) \text { where } \\
& \left(T_{1}\left(x_{1}, \cdots, x_{m}\right)=\sum_{t_{1}=0}^{n_{1}} \cdots \sum_{t_{m}=0}^{n_{m}} f^{\left(p_{1}\right) \cdots\left(p_{m}\right)}\left(\frac{t_{1}}{n_{1}}, \cdots, \frac{t_{m}}{n_{m}}\right)\right. \\
& \times \prod_{i=1}^{m}\left(\begin{array}{c}
n_{i} \\
t_{i}
\end{array}\right) x_{i}^{t_{i}}\left(1-x_{i}\right)^{n_{i}-t_{i}}, \\
& T_{2}\left(x_{1}, \cdots, x_{m}\right)=\sum_{t_{1}=0}^{n_{1}} \cdots \sum_{t_{m}=0}^{n_{m}}\left[f^{\left(p_{1}\right) \cdots\left(p_{m}\right)}\left(\varepsilon_{t_{1}}, \cdots, \varepsilon_{t_{m}}\right)\right. \\
& \left.-f^{\left(p_{1}\right) \cdots\left(p_{m}\right)}\left(\frac{t_{1}}{n_{1}}, \cdots, \frac{t_{m}}{n_{m}}\right)\right] \prod_{i=1}^{m}\left(\begin{array}{c}
n_{i} \\
t_{i}
\end{array}\right) x_{i}^{t_{i}}\left(1-x_{i}\right)^{n_{i}-t_{i}} .
\end{aligned}
$$

Since $\frac{t_{i}}{n_{i}+p_{i}}<\frac{t_{i}}{n_{i}}<\frac{t_{i}+p_{i}}{n_{i}+p_{i}}, i=1, \cdots, m, t_{i}=0, \cdots, n_{i}$, it follows from the bounds on $\varepsilon_{t_{i}}$, $i=1, \cdots, m$, that

$$
-\frac{p_{i}}{n_{i}+p_{i}}=\frac{t_{i}}{n_{i}+p_{i}}-\frac{t_{i}+p_{i}}{n_{i}+p_{i}}<\varepsilon_{t_{i}}-\frac{t_{i}}{n_{i}}<\frac{t_{i}+p_{i}}{n_{i}+p_{i}}-\frac{t_{i}}{n_{i}+p_{i}}=\frac{p_{i}}{n_{i}+p_{i}}
$$

for $i=1, \cdots, m$. This means $\left|\varepsilon_{t_{i}}-\frac{t_{i}}{n_{i}}\right|<\frac{p_{i}}{n_{i}+p_{i}}, \quad i=1, \cdots, m$. From the uniform continuity of $f^{\left(p_{1}\right) \cdots\left(p_{m}\right)}\left(x_{1}, \cdots, x_{m}\right)$, given $\varepsilon>0$, we can find $n_{i 0}$ such that for all $n_{i} \geq n_{i 0}$ and all $t_{i}$,

$$
\left|f^{\left(p_{1}\right) \cdots\left(p_{m}\right)}\left(\varepsilon_{t_{1}}, \cdots, \varepsilon_{t_{m}}\right)-f^{\left(p_{1}\right) \cdots\left(p_{m}\right)}\left(\frac{t_{1}}{n_{1}}, \cdots, \frac{t_{m}}{n_{m}}\right)\right|<\varepsilon .
$$

Thus, $T_{2}\left(x_{1}, \cdots, x_{m}\right)$ converges uniformly to zero on $[0,1]^{m}$. We have 


$$
\prod_{i=1}^{m} \frac{n_{i} !\left(n_{i}+p_{i}\right)^{p_{i}}}{\left(n_{i}+p_{i}\right) !} \longrightarrow 1 \text { as } n_{i} \rightarrow \infty, \forall i=1, \cdots, m
$$

and we see from Theorem 2) with $f^{\left(p_{1}\right) \cdots\left(p_{m}\right)}$ in place of $f$ that $T_{1}\left(x_{1}, \cdots, x_{m}\right)$ converges uniformly to $f^{\left(p_{1}\right) \cdots\left(p_{m}\right)}\left(x_{1}, \cdots, x_{m}\right)$. This completes the proof.

The following theorem, in the case where $f$ is a convex function, is the generalization of the inequality related to the decreasing of the sequence of Bernstein's polynomials with one variable (see References [1,31]).

Theorem 5. Let $f\left(x_{1}, \cdots, x_{m}\right)$ be convex in $[0,1]^{m}$. Then, for $n_{i}=2,3, \cdots, i=1, \cdots, m$, we have

$$
B_{n_{1}-1, \cdots, n_{m}-1}\left(f ; x_{1}, \cdots, x_{m}\right) \geq B_{n_{1}, \cdots, n_{m}}\left(f ; x_{1}, \cdots, x_{m}\right), \quad\left(x_{1}, \cdots, x_{m}\right) \in[0,1]^{m} .
$$

Proof of Theorem 5. The case of one variable is already solved (see P. J. Davis [1]). Because of long calculations and space limitations, we will just show how this theorem works for three variables (we did not choose the case of two variables because with three variables, our strategy is well illustrated and we can easily use it for any large number of variables). Set $t_{i}=\frac{x_{i}}{1-x_{i}}, i=1,2,3$. Then,

$$
\left\{\begin{array} { l } 
{ x _ { i } ^ { k _ { i } } ( 1 - x _ { i } ) ^ { - k _ { i } } = t _ { i } ^ { k _ { i } } } \\
{ 1 + t _ { i } = ( 1 - x _ { i } ) ^ { - 1 } , }
\end{array} \quad \text { that is, } \quad \left\{\begin{array}{l}
x_{i}^{k_{i}}\left(1-x_{i}\right)^{-k_{i}}=t_{i}^{k_{i}} \\
x_{i}^{k_{i}}\left(1-x_{i}\right)^{-k_{i}-1}=t_{i}^{k_{i}}\left(1+t_{i}\right)
\end{array}\right.\right.
$$

and

$$
\begin{aligned}
& \left(1-x_{1}\right)^{-n_{1}}\left(1-x_{2}\right)^{-n_{2}}\left(1-x_{3}\right)^{-n_{3}}\left(B_{n_{1}-1, n_{2}-1, n_{3}-1}\left(f ; x_{1}, x_{2}, x_{3}\right)-B_{n_{1}, n_{2}, n_{3}}\left(f ; x_{1}, x_{2}, x_{3}\right)\right) \\
& =\sum_{k_{1}=0}^{n_{1}-1} \sum_{k_{2}=0}^{n_{2}-1} \sum_{k_{3}=0}^{n_{3}-1} f\left(\frac{k_{1}}{n_{1}-1}, \frac{k_{2}}{n_{2}-1}, \frac{k_{3}}{n_{3}-1}\right) \prod_{i=1}^{3}\left(\begin{array}{c}
n_{i}-1 \\
k_{i}
\end{array}\right) x_{i}^{k_{i}}\left(1-x_{i}\right)^{-k_{i}-1} \\
& -\sum_{k_{1}=0}^{n_{1}} \sum_{k_{2}=0}^{n_{2}} \sum_{k_{3}=0}^{n_{3}} f\left(\frac{k_{1}}{n_{1}}, \frac{k_{2}}{n_{2}}, \frac{k_{3}}{n_{3}}\right) \prod_{i=1}^{3}\left(\begin{array}{c}
n_{i} \\
k_{i}
\end{array}\right) x_{i}^{k_{i}}\left(1-x_{i}\right)^{-k_{i}} \\
& =\sum_{k_{1}=0}^{n_{1}-1} \sum_{k_{2}=0}^{n_{2}-1} \sum_{k_{3}=0}^{n_{3}-1} f\left(\frac{k_{1}}{n_{1}-1}, \frac{k_{2}}{n_{2}-1}, \frac{k_{3}}{n_{3}-1}\right) \prod_{i=1}^{3}\left(\begin{array}{c}
n_{i}-1 \\
k_{i}
\end{array}\right) t_{i}^{k_{i}}\left(1+t_{i}\right) \\
& -\sum_{k_{1}=0}^{n_{1}} \sum_{k_{2}=0}^{n_{2}} \sum_{k_{3}=0}^{n_{3}} f\left(\frac{k_{1}}{n_{1}}, \frac{k_{2}}{n_{2}}, \frac{k_{3}}{n_{3}}\right) \prod_{i=1}^{3}\left(\begin{array}{l}
n_{i} \\
k_{i}
\end{array}\right) t_{i}^{k_{i}} \\
& =\sum_{i_{1}, i_{2}, i_{3} \in\{0,1\}} \sum_{k_{1}=i_{1}}^{n_{1}-1+i_{1}} \sum_{k_{2}=i_{2}}^{n_{2}-1+i_{2}} \sum_{k_{3}=i_{3}}^{n_{3}-1+i_{3}} f\left(\frac{k_{1}-i_{1}}{n_{1}-1}, \frac{k_{2}-i_{2}}{n_{2}-1}, \frac{k_{3}-i_{3}}{n_{3}-1}\right)\left(\begin{array}{l}
n_{1}-1 \\
k_{1}-i_{1}
\end{array}\right)\left(\begin{array}{l}
n_{2}-1 \\
k_{2}-i_{2}
\end{array}\right)\left(\begin{array}{l}
n_{3}-1 \\
k_{3}-i_{3}
\end{array}\right) t_{1}^{k_{1}} t_{2}^{k_{2}} t_{3}^{k_{3}} \\
& -\sum_{k_{1}=0}^{n_{1}} \sum_{k_{2}=0}^{n_{2}} \sum_{k_{3}=0}^{n_{3}} f\left(\frac{k_{1}}{n_{1}}, \frac{k_{2}}{n_{2}}, \frac{k_{3}}{n_{3}}\right)\left(\begin{array}{l}
n_{1} \\
k_{1}
\end{array}\right)\left(\begin{array}{l}
n_{2} \\
k_{2}
\end{array}\right)\left(\begin{array}{l}
n_{3} \\
k_{3}
\end{array}\right) t_{1}^{k_{1}} t_{2}^{k_{2}} t_{3}^{k_{3}} \\
& =\sum_{i_{1}, i_{2}, i_{3} \in\{0,1\}} \sum_{k_{1}=i_{1}}^{n_{1}-1+i_{1}} \sum_{k_{2}=i_{2}}^{n_{2}-1+i_{2}} \sum_{k_{3}=i_{3}}^{n_{3}-1+i_{3}} f\left(\frac{k_{1}-i_{1}}{n_{1}-1}, \frac{k_{2}-i_{2}}{n_{2}-1}, \frac{k_{3}-i_{3}}{n_{3}-1}\right)\left(\begin{array}{l}
n_{1}-1 \\
k_{1}-i_{1}
\end{array}\right)\left(\begin{array}{l}
n_{2}-1 \\
k_{2}-i_{2}
\end{array}\right)\left(\begin{array}{l}
n_{3}-1 \\
k_{3}-i_{3}
\end{array}\right) t_{1}^{k_{1}} t_{2}^{k_{2}} t_{3}^{k_{3}} \\
& -\sum_{k_{1}=0}^{n_{1}} \sum_{k_{2}=0}^{n_{2}} \sum_{k_{3}=0}^{n_{3}} f\left(\frac{k_{1}}{n_{1}}, \frac{k_{2}}{n_{2}}, \frac{k_{3}}{n_{3}}\right)\left(\begin{array}{l}
n_{1} \\
k_{1}
\end{array}\right)\left(\begin{array}{l}
n_{2} \\
k_{2}
\end{array}\right)\left(\begin{array}{l}
n_{3} \\
k_{3}
\end{array}\right) t_{1}^{k_{1}} t_{2}^{k_{2}} t_{3}^{k_{3}} \\
& =\mathrm{F}_{n_{1}, n_{2}, n_{3}}\left(t_{1}, t_{2}, t_{3}\right) \text {. }
\end{aligned}
$$

$\mathrm{F}_{n_{1}, n_{2}, n_{3}}\left(t_{1}, t_{2}, t_{3}\right)$ is a polynomial of the variables $t_{1}, t_{2}$ and $t_{3}$. It can be written as 


$$
\mathrm{F}_{n_{1}, n_{2}, n_{3}}\left(t_{1}, t_{2}, t_{3}\right)=\sum_{k_{1}=0}^{n_{1}} \sum_{k_{2}=0}^{n_{2}} \sum_{k_{3}=0}^{n_{3}} \mathrm{~F}_{n_{1}, n_{2}, n_{3}}^{k_{1}, k_{2}, k_{3}} t_{1}^{k_{1}} t_{2}^{k_{2}} t_{3}^{k_{3}}
$$

To have our result, we just need to prove that $\mathrm{F}_{n_{1}, n_{2}, n_{3}}\left(t_{1}, t_{2}, t_{3}\right)$ is positive.

For $i_{e} \in\{0,1\}$ with $e \in\{1,2,3\}$, we define

$$
\overline{i_{e}}=\left\{\begin{array}{l}
0 \text { if } i_{e}=1 \\
1 \text { if } i_{e}=0
\end{array}\right.
$$

Now, we will analyse all the possibilities of the summand $F_{n_{1}, n_{2}, n_{3}}^{k_{1}, k_{2} k_{3}} t_{1}^{k_{1}} t_{2}^{k_{2}} t_{3}^{k_{3}}$ of our polynomial $\mathrm{F}_{n_{1}, n_{2}, n_{3}}\left(t_{1}, t_{2}, t_{3}\right)$. For simplicity and depending of the upper and lower bounds of (99), we will write $\mathrm{F}_{n_{1}, n_{2}, n_{3}}\left(t_{1}, t_{2}, t_{3}\right)$ as

$$
\mathrm{F}_{n_{1}, n_{2}, n_{3}}\left(t_{1}, t_{2}, t_{3}\right)=\mathrm{F}_{n_{1}, n_{2}, n_{3}}^{1}\left(t_{1}, t_{2}, t_{3}\right)+\mathrm{F}_{n_{1}, n_{2}, n_{3}}^{2}\left(t_{1}, t_{2}, t_{3}\right)+\mathrm{F}_{n_{1}, n_{2}, n_{3}}^{3}\left(t_{1}, t_{2}, t_{3}\right)+\mathrm{F}_{n_{1}, n_{2}, n_{3}}^{4}\left(t_{1}, t_{2}, t_{3}\right),
$$

where

* $\quad \mathrm{F}_{n_{1}, n_{2}, n_{3}}^{1}\left(t_{1}, t_{2}, t_{3}\right)$ is the sum with index $1 \leq k_{1} \leq n_{1}-1,1 \leq k_{2} \leq n_{2}-1$ and $1 \leq k_{3} \leq n_{3}-1$;

* $\quad \mathrm{F}_{n_{1}, n_{2}, n_{3}}^{2}\left(t_{1}, t_{2}, t_{3}\right)$ is the sum where all the index $k_{i}, i \in\{1,2,3\}$ start at 1 , but with just one index reaching the upper bound $n_{i}, i \in\{1,2,3\}$;

* $\quad \mathrm{F}_{n_{1}, n_{2}, n_{3}}^{3}\left(t_{1}, t_{2}, t_{3}\right)$ is the sum where all the index $k_{i}$ are less or equal to $n_{i}-1, i \in\{1,2,3\}$, but just one index $k_{i}, i \in\{1,2,3\}$ reaching the lower bound 0 ;

* $\quad \mathrm{F}_{n_{1}, n_{2}, n_{3}}^{4}\left(t_{1}, t_{2}, t_{3}\right)$ is the rest of the sum in Equation (99) after removing the terms $\mathrm{F}_{n_{1}, n_{2}, n_{3}}^{1}\left(t_{1}, t_{2}, t_{3}\right)$, $\mathrm{F}_{n_{1}, n_{2}, n_{3}}^{2}\left(t_{1}, t_{2}, t_{3}\right)$ and $\mathrm{F}_{n_{1}, n_{2}, n_{3}}^{3}\left(t_{1}, t_{2}, t_{3}\right)$.

- $\quad$ Case 1: First, we take the sum with index $1 \leq k_{1} \leq n_{1}-1,1 \leq k_{2} \leq n_{2}-1$ and $1 \leq k_{3} \leq n_{3}-1$ in Equation (99). We obtain

$$
\begin{aligned}
& \mathrm{F}_{n_{1}, n_{2}, n_{3}}^{1}\left(t_{1}, t_{2}, t_{3}\right) \\
= & \sum_{k_{1}=1}^{n_{1}-1} \sum_{k_{2}=1}^{1} \sum_{k_{3}=1}^{n_{3}-1}\left(\sum_{i_{1}, i_{2}, i_{3} \in\{0,1\}} f\left(\frac{k_{1}-i_{1}}{n_{1}-1}, \frac{k_{2}-i_{2}}{n_{2}-1}, \frac{k_{3}-i_{3}}{n_{3}-1}\right)\left(\begin{array}{l}
n_{1}-1 \\
k_{1}-i_{1}
\end{array}\right)\left(\begin{array}{l}
n_{2}-1 \\
k_{2}-i_{2}
\end{array}\right)\left(\begin{array}{l}
n_{3}-1 \\
k_{3}-i_{3}
\end{array}\right)\right. \\
& \left.-f\left(\frac{k_{1}}{n_{1}}, \frac{k_{2}}{n_{2}}, \frac{k_{3}}{n_{3}}\right)\left(\begin{array}{l}
n_{1} \\
k_{1}
\end{array}\right)\left(\begin{array}{l}
n_{2} \\
k_{2}
\end{array}\right)\left(\begin{array}{l}
n_{3} \\
k_{3}
\end{array}\right)\right) t_{1}^{k_{1}} t_{2}^{k_{2}} t_{3}^{k_{3}} .
\end{aligned}
$$

Next, we add and subtract the following expression in $\mathrm{F}_{n_{1}, n_{2}, n_{3}}^{1}\left(t_{1}, t_{2}, t_{3}\right)$ to obtain:

$$
\begin{aligned}
& \sum_{k_{1}=1}^{n_{1}-1} \sum_{k_{2}=1}^{n_{2}-1} \sum_{k_{3}=1}^{n_{3}-1}\left(\sum_{i_{1}, i_{2} \in\{0,1\}} f\left(\frac{k_{1}-i_{1}}{n_{1}-1}, \frac{k_{2}-i_{2}}{n_{2}-1}, \frac{k_{3}}{n_{3}}\right)\left(\begin{array}{l}
n_{1}-1 \\
k_{1}-i_{1}
\end{array}\right)\left(\begin{array}{l}
n_{2}-1 \\
k_{2}-i_{2}
\end{array}\right)\left(\begin{array}{l}
n_{3} \\
k_{3}
\end{array}\right)\right. \\
& \left.+\sum_{i_{1} \in\{0,1\}} f\left(\frac{k_{1}-i_{1}}{n_{1}-1}, \frac{k_{2}}{n_{2}}, \frac{k_{3}}{n_{3}}\right)\left(\begin{array}{l}
n_{1}-1 \\
k_{1}-i_{1}
\end{array}\right)\left(\begin{array}{l}
n_{2} \\
k_{2}
\end{array}\right)\left(\begin{array}{l}
n_{3} \\
k_{3}
\end{array}\right)\right) t_{1}^{k_{1}} t_{2}^{k_{2}} t_{3}^{k_{3}} .
\end{aligned}
$$

Next, we group now the terms in $\mathrm{F}_{n_{1}, n_{2}, n_{3}}^{1}\left(t_{1}, t_{2}, t_{3}\right)$ given by the previous expression in the following three blocks: $\mathrm{F}_{n_{1}, n_{2}, n_{3}}^{1}\left(t_{1}, t_{2}, t_{3}\right)=B_{1}+B_{2}+B_{3}$ with 


$$
\begin{aligned}
& B_{1}=\sum_{k_{1}=1}^{n_{1}-1} \sum_{k_{2}=1}^{n_{2}-1} \sum_{k_{3}=1}^{n_{3}-1}\left(\sum _ { i _ { 1 } , i _ { 2 } , i _ { 3 } \in \{ 0 , 1 \} } \left(f\left(\frac{k_{1}-i_{1}}{n_{1}-1}, \frac{k_{2}-i_{2}}{n_{2}-1}, \frac{k_{3}-i_{3}}{n_{3}-1}\right)\left(\begin{array}{l}
n_{1}-1 \\
k_{1}-i_{1}
\end{array}\right)\left(\begin{array}{l}
n_{2}-1 \\
k_{2}-i_{2}
\end{array}\right)\left(\begin{array}{l}
n_{3}-1 \\
k_{3}-i_{3}
\end{array}\right)\right.\right. \\
& +f\left(\frac{k_{1}-i_{1}}{n_{1}-1}, \frac{k_{2}-i_{2}}{n_{2}-1}, \frac{k_{3}-\overline{i_{3}}}{n_{3}-1}\right)\left(\begin{array}{l}
n_{1}-1 \\
k_{1}-i_{1}
\end{array}\right)\left(\begin{array}{l}
n_{2}-1 \\
k_{2}-i_{2}
\end{array}\right)\left(\begin{array}{l}
n_{3}-1 \\
k_{3}-\overline{i_{3}}
\end{array}\right) \\
& \left.\left.-f\left(\frac{k_{1}-i_{1}}{n_{1}-1}, \frac{k_{2}-i_{2}}{n_{2}-1}, \frac{k_{3}}{n_{3}}\right)\left(\begin{array}{c}
n_{1}-1 \\
k_{1}-i_{1}
\end{array}\right)\left(\begin{array}{l}
n_{2}-1 \\
k_{2}-i_{2}
\end{array}\right)\left(\begin{array}{l}
n_{3} \\
k_{3}
\end{array}\right)\right)\right) t_{1}^{k_{1}} t_{2}^{k_{2}} t_{3}^{k_{3}}, \\
& B_{2}=\sum_{k_{1}=1}^{n_{1}-1} \sum_{k_{2}=1}^{n_{2}-1} \sum_{k_{3}=1}^{n_{3}-1}\left(\sum _ { i _ { 1 } , i _ { 2 } \in \{ 0 , 1 \} } \left(f\left(\frac{k_{1}-i_{1}}{n_{1}-1}, \frac{k_{2}-i_{2}}{n_{2}-1}, \frac{k_{3}}{n_{3}}\right)\left(\begin{array}{l}
n_{1}-1 \\
k_{1}-i_{1}
\end{array}\right)\left(\begin{array}{l}
n_{2}-1 \\
k_{2}-i_{2}
\end{array}\right)\left(\begin{array}{l}
n_{3} \\
k_{3}
\end{array}\right)\right.\right. \\
& +f\left(\frac{k_{1}-i_{1}}{n_{1}-1}, \frac{k_{2}-\overline{i_{2}}}{n_{2}-1}, \frac{k_{3}}{n_{3}}\right)\left(\begin{array}{l}
n_{1}-1 \\
k_{1}-i_{1}
\end{array}\right)\left(\begin{array}{l}
n_{2}-1 \\
k_{2}-\overline{i_{2}}
\end{array}\right)\left(\begin{array}{l}
n_{3} \\
k_{3}
\end{array}\right) \\
& \left.\left.-f\left(\frac{k_{1}-i_{1}}{n_{1}-1}, \frac{k_{2}}{n_{2}}, \frac{k_{3}}{n_{3}}\right)\left(\begin{array}{l}
n_{1}-1 \\
k_{1}-i_{1}
\end{array}\right)\left(\begin{array}{l}
n_{2} \\
k_{2}
\end{array}\right)\left(\begin{array}{l}
n_{3} \\
k_{3}
\end{array}\right)\right)\right) t_{1}^{k_{1}} t_{2}^{k_{2}} t_{3}^{k_{3}}, \\
& B_{3}=\sum_{k_{1}=1}^{n_{1}-1} \sum_{k_{2}=1}^{n_{2}-1} \sum_{k_{3}=1}^{n_{3}-1}\left(\sum _ { i _ { 1 } \in \{ 0 , 1 \} } \left(f\left(\frac{k_{1}-i_{1}}{n_{1}-1}, \frac{k_{2}}{n_{2}}, \frac{k_{3}}{n_{3}}\right)\left(\begin{array}{l}
n_{1}-1 \\
k_{1}-i_{1}
\end{array}\right)\left(\begin{array}{l}
n_{2} \\
k_{2}
\end{array}\right)\left(\begin{array}{l}
n_{3} \\
k_{3}
\end{array}\right)\right.\right. \\
& +f\left(\frac{k_{1}-\overline{i_{1}}}{n_{1}-1}, \frac{k_{2}}{n_{2}}, \frac{k_{3}}{n_{3}}\right)\left(\begin{array}{l}
n_{1}-1 \\
k_{1}-\overline{i_{1}}
\end{array}\right)\left(\begin{array}{l}
n_{2} \\
k_{2}
\end{array}\right)\left(\begin{array}{l}
n_{3} \\
k_{3}
\end{array}\right) \\
& \left.\left.-f\left(\frac{k_{1}}{n_{1}}, \frac{k_{2}}{n_{2}}, \frac{k_{3}}{n_{3}}\right)\left(\begin{array}{l}
n_{1} \\
k_{1}
\end{array}\right)\left(\begin{array}{l}
n_{2} \\
k_{2}
\end{array}\right)\left(\begin{array}{l}
n_{3} \\
k_{3}
\end{array}\right)\right)\right) t_{1}^{k_{1}} t_{2}^{k_{2}} t_{3}^{k_{3}} \text {. }
\end{aligned}
$$

We are going to show that $B_{i} \geq 0$ for $i \in\{1,2,3\}$. It is obvious that

$$
\left(\begin{array}{c}
n-1 \\
k
\end{array}\right)=\left(\begin{array}{l}
n \\
k
\end{array}\right) \frac{n-k}{n}(n \geq k+1) \text { and }\left(\begin{array}{l}
n-1 \\
k-1
\end{array}\right)=\left(\begin{array}{l}
n \\
k
\end{array}\right) \frac{k}{n}(n \geq k) \text { with } k, n \text { positive integers. }
$$

Now, using Equation (107) in each $B_{i}$ we get

$$
\begin{aligned}
B_{1}= & \sum_{k_{1}=1}^{n_{1}-1} \sum_{k_{2}=1}^{n_{2}-1} \sum_{k_{3}=1}^{n_{3}-1}\left(\sum _ { i _ { 1 } , i _ { 2 } \in \{ 0 , 1 \} } ( \begin{array} { c } 
{ n _ { 1 } - 1 } \\
{ k _ { 1 } - i _ { 1 } }
\end{array} ) ( \begin{array} { l } 
{ n _ { 2 } - 1 } \\
{ k _ { 2 } - i _ { 2 } }
\end{array} ) ( \begin{array} { l } 
{ n _ { 3 } } \\
{ k _ { 3 } }
\end{array} ) \left(\frac{n_{3}-k_{3}}{n_{3}} f\left(\frac{k_{1}-i_{1}}{n_{1}-1}, \frac{k_{2}-i_{2}}{n_{2}-1}, \frac{k_{3}}{n_{3}-1}\right)\right.\right. \\
& \left.+\frac{k_{3}}{n_{3}} f\left(\frac{k_{1}-i_{1}}{n_{1}-1}, \frac{k_{2}-i_{2}}{n_{2}-1}, \frac{k_{3}-1}{n_{3}-1}\right)-f\left(\frac{k_{1}-i_{1}}{n_{1}-1}, \frac{k_{2}-i_{2}}{n_{2}-1}, \frac{k_{3}}{n_{3}}\right)\right) \\
& +\sum_{i_{1}, i_{2} \in\{0,1\}}\left(\begin{array}{l}
n_{1}-1 \\
k_{1}-i_{1}
\end{array}\right)\left(\begin{array}{l}
n_{2}-1 \\
k_{2}-i_{2}
\end{array}\right)\left(\begin{array}{l}
n_{3} \\
k_{3}
\end{array}\right)\left(\frac{k_{3}}{n_{3}} f\left(\frac{k_{1}-i_{1}}{n_{1}-1}, \frac{k_{2}-i_{2}}{n_{2}-1}, \frac{k_{3}-1}{n_{3}-1}\right)\right. \\
& \left.\left.+\frac{n_{3}-k_{3}}{n_{3}} f\left(\frac{k_{1}-i_{1}}{n_{1}-1}, \frac{k_{2}-i_{2}}{n_{2}-1}, \frac{k_{3}}{n_{3}-1}\right)-f\left(\frac{k_{1}-i_{1}}{n_{1}-1}, \frac{k_{2}-i_{2}}{n_{2}-1}, \frac{k_{3}}{n_{3}}\right)\right)\right) t_{1}^{k_{1}} t_{2}^{k_{2}} t_{3}^{k_{3}},
\end{aligned}
$$




$$
\begin{aligned}
B_{2}= & \sum_{k_{1}=1}^{n_{1}-1} \sum_{k_{2}=1}^{n_{2}-1} \sum_{k_{3}=1}^{n_{3}-1}\left(\sum _ { i _ { 1 } \in \{ 0 , 1 \} } ( \begin{array} { l } 
{ n _ { 1 } - 1 } \\
{ k _ { 1 } - i _ { 1 } }
\end{array} ) ( \begin{array} { l } 
{ n _ { 2 } } \\
{ k _ { 2 } }
\end{array} ) ( \begin{array} { l } 
{ n _ { 3 } } \\
{ k _ { 3 } }
\end{array} ) \left(\frac{n_{2}-k_{2}}{n_{2}} f\left(\frac{k_{1}-i_{1}}{n_{1}-1}, \frac{k_{2}}{n_{2}-1}, \frac{k_{3}}{n_{3}}\right)\right.\right. \\
& \left.+\frac{k_{2}}{n_{2}} f\left(\frac{k_{1}-i_{1}}{n_{1}-1}, \frac{k_{2}-1}{n_{2}-1}, \frac{k_{3}}{n_{3}}\right)-f\left(\frac{k_{1}-i_{1}}{n_{1}-1}, \frac{k_{2}}{n_{2}}, \frac{k_{3}}{n_{3}}\right)\right) \\
& +\sum_{i_{1} \in\{0,1\}}\left(\begin{array}{l}
n_{1}-1 \\
k_{1}-i_{1}
\end{array}\right)\left(\begin{array}{l}
n_{2} \\
k_{2}
\end{array}\right)\left(\begin{array}{l}
n_{3} \\
k_{3}
\end{array}\right)\left(\frac{k_{2}}{n_{2}} f\left(\frac{k_{1}-i_{1}}{n_{1}-1}, \frac{k_{2}-1}{n_{2}-1}, \frac{k_{3}}{n_{3}}\right)\right. \\
& \left.\left.+\frac{n_{2}-k_{2}}{n_{2}} f\left(\frac{k_{1}-i_{1}}{n_{1}-1}, \frac{k_{2}}{n_{2}-1}, \frac{k_{3}}{n_{3}}\right)-f\left(\frac{k_{1}-i_{1}}{n_{1}-1}, \frac{k_{2}}{n_{2}}, \frac{k_{3}}{n_{3}}\right)\right)\right) t_{1}^{k_{1}} t_{2}^{k_{2}} t_{3}^{k_{3}}, \\
B_{3}= & \sum_{k_{1}=1}^{n_{1}-1} \sum_{k_{2}=1}^{n_{2}-1} \sum_{k_{3}=1}^{n_{3}-1}\left(( \begin{array} { l } 
{ n _ { 1 } } \\
{ k _ { 1 } }
\end{array} ) ( \begin{array} { l } 
{ n _ { 2 } } \\
{ k _ { 2 } }
\end{array} ) ( \begin{array} { l } 
{ n _ { 3 } } \\
{ k _ { 3 } }
\end{array} ) \left(\frac{n_{1}-k_{1}}{n_{1}} f\left(\frac{k_{1}}{n_{1}-1}, \frac{k_{2}}{n_{2}}, \frac{k_{3}}{n_{3}}\right)\right.\right. \\
& \left.+\frac{k_{1}}{n_{1}} f\left(\frac{k_{1}-1}{n_{1}-1}, \frac{k_{2}}{n_{2}}, \frac{k_{3}}{n_{3}}\right)-f\left(\frac{k_{1}}{n_{1}}, \frac{k_{2}}{n_{2}}, \frac{k_{3}}{n_{3}}\right)\right) \\
& +\left(\begin{array}{l}
n_{1} \\
k_{1}
\end{array}\right)\left(\begin{array}{l}
n_{2} \\
k_{2}
\end{array}\right)\left(\begin{array}{l}
n_{3} \\
k_{3}
\end{array}\right)\left(\frac{k_{1}}{n_{1}} f\left(\frac{k_{1}-1}{n_{1}-1}, \frac{k_{2}}{n_{2}}, \frac{k_{3}}{n_{3}}\right)\right. \\
& \left.\left.+\frac{n_{1}-k_{1}}{n_{1}} f\left(\frac{k_{1}}{n_{1}-1}, \frac{k_{2}}{n_{2}}, \frac{k_{3}}{n_{3}}\right)-f\left(\frac{k_{1}}{n_{1}}, \frac{k_{2}}{n_{2}}, \frac{k_{3}}{n_{3}}\right)\right)\right) t_{1}^{k_{1}} t_{2}^{k_{2}} t_{3}^{k_{3}},
\end{aligned}
$$

which implies that $B_{i} \geq 0, i \in\{1,2,3\}$ by the convexity of $f$ and the fact that $t_{1}, t_{2}$ and $t_{3}$ are also positive.

- Case 2: We take the sum in Equation (99), where all the index start at 1 , but just one index $k_{i}$, $i \in\{1,2,3\}$ reaches the upper bound $n_{i}, i \in\{1,2,3\}$. This corresponds to $F_{n_{1}, n_{2}, n_{3}}^{2}\left(t_{1}, t_{2}, t_{3}\right)$ which can be written as

$$
\mathrm{F}_{n_{1}, n_{2}, n_{3}}^{2}\left(t_{1}, t_{2}, t_{3}\right)=\mathrm{F}_{n_{1}, n_{2}, n_{3}}^{2,1}\left(t_{1}, t_{2}, t_{3}\right)+\mathrm{F}_{n_{1}, n_{2}, n_{3}}^{2,2}\left(t_{1}, t_{2}, t_{3}\right)+\mathrm{F}_{n_{1}, n_{2}, n_{3}}^{2,3}\left(t_{1}, t_{2}, t_{3}\right)
$$

where $\mathrm{F}_{n_{1}, n_{2}, n_{3}}^{2, i}\left(t_{1}, t_{2}, t_{3}\right)$ is the sum where the index $k_{i}$ reaches the upper bound $n_{i}$ for $i \in\{1,2,3\}$. We have to show that $\mathrm{F}_{n_{1}, n_{2}, n_{3}}^{2, i}\left(t_{1}, t_{2}, t_{3}\right), i \in\{1,2,3\}$, are positive. Let us start with $\mathrm{F}_{n_{1}, n_{2}, n_{3}}^{2,1}\left(t_{1}, t_{2}, t_{3}\right)$ :

$$
\begin{aligned}
\mathrm{F}_{n_{1}, n_{2}, n_{3}}^{2,1}\left(t_{1}, t_{2}, t_{3}\right)= & \sum_{k_{2}=1}^{n_{2}-1} \sum_{k_{3}=1}^{n_{3}-1}\left(\sum_{i_{2}, i_{3} \in\{0,1\}} f\left(1, \frac{k_{2}-i_{2}}{n_{2}-1}, \frac{k_{3}-i_{3}}{n_{3}-1}\right)\left(\begin{array}{l}
n_{2}-1 \\
k_{2}-i_{2}
\end{array}\right)\left(\begin{array}{l}
n_{3}-1 \\
k_{3}-i_{3}
\end{array}\right)\right. \\
& \left.-f\left(1, \frac{k_{2}}{n_{2}}, \frac{k_{3}}{n_{3}}\right)\left(\begin{array}{l}
n_{2} \\
k_{2}
\end{array}\right)\left(\begin{array}{l}
n_{3} \\
k_{3}
\end{array}\right)\right) t_{1}^{n_{1}} t_{2}^{k_{2}} t_{3}^{k_{3}} .
\end{aligned}
$$

We add and subtract the following expression in $\mathrm{F}_{n_{1}, n_{2}, n_{3}}^{2,1}\left(t_{1}, t_{2}, t_{3}\right)$

$$
\sum_{k_{2}=1}^{n_{2}-1} \sum_{k_{3}=1}^{n_{3}-1}\left(\sum_{i_{2} \in\{0,1\}} f\left(1, \frac{k_{2}-i_{2}}{n_{2}-1}, \frac{k_{3}}{n_{3}}\right)\left(\begin{array}{l}
n_{2}-1 \\
k_{2}-i_{2}
\end{array}\right)\left(\begin{array}{l}
n_{3} \\
k_{3}
\end{array}\right)\right) t_{1}^{n_{1}} t_{2}^{k_{2}} t_{3}^{k_{3}} .
$$

We now group the terms in $\mathrm{F}_{n_{1}, n_{2}, n_{3}}^{2,1}\left(t_{1}, t_{2}, t_{3}\right)$ in the following two blocks: $\mathrm{F}_{n_{1}, n_{2}, n_{3}}^{2,1}\left(t_{1}, t_{2}, t_{3}\right)=B_{1}+B_{2}$ with 


$$
\begin{aligned}
B_{1}= & \sum_{k_{2}=1}^{n_{2}-1} \sum_{k_{3}=1}^{n_{3}-1}\left(\sum _ { i _ { 2 } , i _ { 3 } \in \{ 0 , 1 \} } \left(f\left(1, \frac{k_{2}-i_{2}}{n_{2}-1}, \frac{k_{3}-i_{3}}{n_{3}-1}\right)\left(\begin{array}{l}
n_{2}-1 \\
k_{2}-i_{2}
\end{array}\right)\left(\begin{array}{l}
n_{3}-1 \\
k_{3}-i_{3}
\end{array}\right)\right.\right. \\
& +f\left(1, \frac{k_{2}-i_{2}}{n_{2}-1}, \frac{k_{3}-\overline{i_{3}}}{n_{3}-1}\right)\left(\begin{array}{l}
n_{2}-1 \\
k_{2}-i_{2}
\end{array}\right)\left(\begin{array}{l}
n_{3}-1 \\
k_{3}-\overline{i_{3}}
\end{array}\right) \\
& \left.\left.-f\left(1, \frac{k_{2}-i_{2}}{n_{2}-1}, \frac{k_{3}}{n_{3}}\right)\left(\begin{array}{l}
n_{2}-1 \\
k_{2}-i_{2}
\end{array}\right)\left(\begin{array}{l}
n_{3} \\
k_{3}
\end{array}\right)\right)\right) t_{1}^{n_{1}} t_{2}^{k_{2}} t_{3}^{k_{3}}, \\
B_{2}= & \sum_{k_{2}=1}^{n_{2}-1} \sum_{k_{3}=1}^{n_{3}-1}\left(\sum _ { i _ { 2 } \in \{ 0 , 1 \} } \left(f\left(1, \frac{k_{2}-i_{2}}{n_{2}-1}, \frac{k_{3}}{n_{3}}\right)\left(\begin{array}{l}
n_{2}-1 \\
k_{2}-i_{2}
\end{array}\right)\left(\begin{array}{l}
n_{3} \\
k_{3}
\end{array}\right)\right.\right. \\
& \left.\left.+f\left(1, \frac{k_{2}-\overline{i_{2}}}{n_{2}-1}, \frac{k_{3}}{n_{3}}\right)\left(\begin{array}{l}
n_{2}-1 \\
k_{2}-\overline{i_{2}}
\end{array}\right)\left(\begin{array}{l}
n_{3} \\
k_{3}
\end{array}\right)-f\left(1, \frac{k_{2}}{n_{2}}, \frac{k_{3}}{n_{3}}\right)\left(\begin{array}{l}
n_{2} \\
k_{2}
\end{array}\right)\left(\begin{array}{l}
n_{3} \\
k_{3}
\end{array}\right)\right)\right) t_{1}^{n_{1}} t_{2}^{k_{2}} t_{3}^{k_{3}} .
\end{aligned}
$$

We are going to show that $B_{i} \geq 0$ for $i \in\{1,2\}$. Using (107) in each $B_{i}$, we get

$$
\begin{aligned}
B_{1}= & \sum_{k_{2}=1}^{n_{2}-1} \sum_{k_{3}=1}^{n_{3}-1}\left(\sum _ { i _ { 2 } \in \{ 0 , 1 \} } ( \begin{array} { l } 
{ n _ { 2 } - 1 } \\
{ k _ { 2 } - i _ { 2 } }
\end{array} ) ( \begin{array} { l } 
{ n _ { 3 } } \\
{ k _ { 3 } }
\end{array} ) \left(\frac{n_{3}-k_{3}}{n_{3}} f\left(1, \frac{k_{2}-i_{2}}{n_{2}-1}, \frac{k_{3}}{n_{3}-1}\right)\right.\right. \\
& \left.+\frac{k_{3}}{n_{3}} f\left(1, \frac{k_{2}-i_{2}}{n_{2}-1}, \frac{k_{3}-1}{n_{3}-1}\right)-f\left(1, \frac{k_{2}-i_{2}}{n_{2}-1}, \frac{k_{3}}{n_{3}}\right)\right) \\
& +\sum_{i_{2} \in\{0,1\}}\left(\begin{array}{l}
n_{2}-1 \\
k_{2}-i_{2}
\end{array}\right)\left(\begin{array}{l}
n_{3} \\
k_{3}
\end{array}\right)\left(\frac{k_{3}}{n_{3}} f\left(1, \frac{k_{2}-i_{2}}{n_{2}-1}, \frac{k_{3}-1}{n_{3}-1}\right)\right. \\
& \left.\left.+\frac{n_{3}-k_{3}}{n_{3}} f\left(1, \frac{k_{2}-i_{2}}{n_{2}-1}, \frac{k_{3}}{n_{3}-1}\right)-f\left(1, \frac{k_{2}-i_{2}}{n_{2}-1}, \frac{k_{3}}{n_{3}}\right)\right)\right) t_{1}^{n_{1}} t_{2}^{k_{2}} t_{3}^{k_{3}}, \\
B_{2}= & \sum_{k_{2}=1}^{n_{2}-1} \sum_{k_{3}=1}^{n_{3}-1}\left(( \begin{array} { l } 
{ n _ { 2 } } \\
{ k _ { 2 } }
\end{array} ) ( \begin{array} { l } 
{ n _ { 3 } } \\
{ k _ { 3 } }
\end{array} ) \left(\frac{n_{2}-k_{2}}{n_{2}} f\left(1, \frac{k_{2}}{n_{2}-1}, \frac{k_{3}}{n_{3}}\right)+\frac{k_{2}}{n_{2}} f\left(1, \frac{k_{2}-1}{n_{2}-1}, \frac{k_{3}}{n_{3}}\right)\right.\right. \\
& \left.\left.-f\left(1, \frac{k_{2}}{n_{2}}, \frac{k_{3}}{n_{3}}\right)\right)\right) t_{1}^{n_{1}} t_{2}^{k_{2}} t_{3}^{k_{3}},
\end{aligned}
$$

which implies that $B_{i} \geq 0, i \in\{1,2\}$ by the convexity of $f$ and the fact that $t_{1}, t_{2}$ and $t_{3}$ are also positive.

Using a similar reasoning as for $\mathrm{F}_{n_{1}, n_{2}, n_{3}}^{2,1}\left(t_{1}, t_{2}, t_{3}\right)$, one shows that $\mathrm{F}_{n_{1}, n_{2}, n_{3}}^{2,2}\left(t_{1}, t_{2}, t_{3}\right)$ and $\mathrm{F}_{n_{1}, n_{2}, n_{3}}^{2,}\left(t_{1}, t_{2}, t_{3}\right)$ are also positive. The main difference appears in the expression that we add and subtract:

(i) for $\mathrm{F}_{n_{1}, n_{2}, n_{3}}^{2,}\left(t_{1}, t_{2}, t_{3}\right)$, the added and subtracted expression is

$$
\sum_{k_{1}=1}^{n_{1}-1} \sum_{k_{3}=1}^{n_{3}-1}\left(\sum_{i_{1} \in\{0,1\}} f\left(\frac{k_{1}-i_{1}}{n_{1}-1}, 1, \frac{k_{3}}{n_{3}}\right)\left(\begin{array}{l}
n_{1}-1 \\
k_{1}-i_{1}
\end{array}\right)\left(\begin{array}{l}
n_{3} \\
k_{3}
\end{array}\right)\right) t_{1}^{k_{1}} t_{2}^{n_{2}} t_{3}^{k_{3}} ;
$$

(ii) for $\mathrm{F}_{n_{1}, n_{2}, n_{3}}^{2,3}\left(t_{1}, t_{2}, t_{3}\right)$, the added and subtracted expression is

$$
\sum_{k_{1}=1}^{n_{1}-1} \sum_{k_{2}=1}^{n_{2}-1}\left(\sum_{i_{1} \in\{0,1\}} f\left(\frac{k_{1}-i_{1}}{n_{1}-1}, \frac{k_{2}}{n_{2}}, 1\right)\left(\begin{array}{l}
n_{1}-1 \\
k_{1}-i_{1}
\end{array}\right)\left(\begin{array}{l}
n_{2} \\
k_{2}
\end{array}\right)\right) t_{1}^{k_{1}} t_{2}^{k_{2}} t_{3}^{n_{3}} .
$$


- Case 3: We take the sum in Equation (99), where all the index $k_{i}$ are less or equal to $n_{i}-1, i \in\{1,2,3\}$, but just one index $k_{i}, i \in\{1,2,3\}$ reaching the lower bound 0 . This corresponds to $\mathrm{F}_{n_{1}, n_{2}, n_{3}}^{3}\left(t_{1}, t_{2}, t_{3}\right)$ which can be written as

$$
\mathrm{F}_{n_{1}, n_{2}, n_{3}}^{3}\left(t_{1}, t_{2}, t_{3}\right)=\mathrm{F}_{n_{1}, n_{2}, n_{3}}^{3,1}\left(t_{1}, t_{2}, t_{3}\right)+\mathrm{F}_{n_{1}, n_{2}, n_{3}}^{3,2}\left(t_{1}, t_{2}, t_{3}\right)+\mathrm{F}_{n_{1}, n_{2}, n_{3}}^{3,3}\left(t_{1}, t_{2}, t_{3}\right),
$$

where $\mathrm{F}_{n_{1}, n_{2}, n_{3}}^{3, i}\left(t_{1}, t_{2}, t_{3}\right)$ is the sum where the index $k_{i}$ reaches the lower bound 0 for $i \in\{1,2,3\}$. We have

$$
\left\{\begin{aligned}
\mathrm{F}_{n_{1}, n_{2}, n_{3}}^{3,1}\left(t_{1}, t_{2}, t_{3}\right) & =\sum_{k_{2}=1}^{n_{2}-1} \sum_{k_{3}=1}^{n_{3}-1} \mathrm{~F}_{n_{1}, n_{2}, n_{3}}^{0, k_{2}, k_{3}} t_{2}^{k_{2}} t_{3}^{k_{3}} ; \\
\mathrm{F}_{n_{1}, n_{2}, n_{3}}^{3,1}\left(t_{1}, t_{2}, t_{3}\right) & =\sum_{k_{1}=1}^{n_{1}-1} \sum_{k_{3}=1}^{n_{3}-1} \mathrm{~F}_{n_{1}, n_{2}, n_{3}}^{k_{1}, 0, k_{3}} t_{1}^{k_{1}} t_{3}^{k_{3}} ; \\
\mathrm{F}_{n_{1}, n_{2}, n_{3}}^{3,1}\left(t_{1}, t_{2}, t_{3}\right) & =\sum_{k_{1}=1}^{n_{1}-1} \sum_{k_{2}=1}^{n_{2}-1} \mathrm{~F}_{n_{1}, n_{2}, n_{3}}^{k_{1}, k_{2}, 0} t_{1}^{k_{1}} t_{2}^{k_{2}}
\end{aligned}\right.
$$

Using a similar reasoning as in case 2 , one shows that $\mathrm{F}_{n_{1}, n_{2}, n_{3}}^{3, i}\left(t_{1}, t_{2}, t_{3}\right), i \in\{1,2,3\}$, are positive. The main difference appears in the expression that we add and subtract:

$$
\begin{aligned}
& \text { for } \mathrm{F}_{n_{1}, n_{2}, n_{3}}^{3,}\left(t_{1}, t_{2}, t_{3}\right) \text {, the added and subtracted expression is } \\
& \qquad \sum_{k_{2}=1}^{n_{2}-1} \sum_{k_{3}=1}^{n_{3}-1}\left(\sum_{i_{2} \in\{0,1\}} f\left(0, \frac{k_{2}-i_{2}}{n_{2}-1}, \frac{k_{3}}{n_{3}}\right)\left(\begin{array}{l}
n_{2}-1 \\
k_{2}-i_{2}
\end{array}\right)\left(\begin{array}{l}
n_{3} \\
k_{3}
\end{array}\right)\right) t_{2}^{k_{2}} t_{3}^{k_{3}} ;
\end{aligned}
$$

(ii) for $\mathrm{F}_{n_{1}, n_{2}, n_{3}}^{3,2}\left(t_{1}, t_{2}, t_{3}\right)$, the added and subtracted expression is

$$
\sum_{k_{1}=1}^{n_{1}-1} \sum_{k_{3}=1}^{n_{3}-1}\left(\sum_{i_{1} \in\{0,1\}} f\left(\frac{k_{1}-i_{1}}{n_{2}-1}, 0, \frac{k_{3}}{n_{3}}\right)\left(\begin{array}{l}
n_{1}-1 \\
k_{1}-i_{1}
\end{array}\right)\left(\begin{array}{l}
n_{3} \\
k_{3}
\end{array}\right)\right) t_{1}^{k_{1}} t_{3}^{k_{3}}
$$

(iii) for $\mathrm{F}_{n_{1}, n_{2}, n_{3}}^{3,3}\left(t_{1}, t_{2}, t_{3}\right)$, the added and subtracted expression is

$$
\sum_{k_{1}=1}^{n_{1}-1} \sum_{k_{2}=1}^{n_{2}-1}\left(\sum_{i_{3} \in\{0,1\}} f\left(\frac{k_{1}-i_{1}}{n_{2}-1}, \frac{k_{2}}{n_{2}}, 0\right)\left(\begin{array}{l}
n_{1}-1 \\
k_{1}-i_{1}
\end{array}\right)\left(\begin{array}{l}
n_{2} \\
k_{2}
\end{array}\right)\right) t_{1}^{k_{1}} t_{2}^{k_{2}}
$$

- Case 4: We take in Equation (99) the rest of the sum after removing $\mathrm{F}_{n_{1}, n_{2}, n_{3}}^{1}\left(t_{1}, t_{2}, t_{3}\right)$, $\mathrm{F}_{n_{1}, n_{2}, n_{3}}^{2}\left(t_{1}, t_{2}, t_{3}\right)$ and $\mathrm{F}_{n_{1}, n_{2}, n_{3}}^{3}\left(t_{1}, t_{2}, t_{3}\right)$. This corresponds to $\mathrm{F}_{n_{1}, n_{2}, n_{3}}^{4}\left(t_{1}, t_{2}, t_{3}\right)$ and has all its terms related with the following monomials: $t_{e}^{k_{e}} t_{j}^{n_{j}} t_{p}^{n_{p}}, t_{e}^{n_{e}} t_{j}^{n_{j}} t_{p}^{n_{p}}, t_{j}^{0} t_{e}^{0} t_{p}^{0}, t_{e}^{k_{e}} t_{j}^{n_{j}}, t_{j}^{k_{j}}$ in (99) where $e, j, p \in$ $\{1,2,3\}, e \neq j, e \neq p, j \neq p$, and $k_{e} \in\left\{1, \cdots, n_{e}-1\right\}$. In this case we do not need to add and subtract some expressions in $\mathrm{F}_{n_{1}, n_{2}, n_{3}}^{4}\left(t_{1}, t_{2}, t_{3}\right)$ which can be written as 


$$
\begin{aligned}
& \mathrm{F}_{n_{1}, n_{2}, n_{3}}^{4}\left(t_{1}, t_{2}, t_{3}\right) \\
& =\sum_{k_{1}=1}^{n_{1}-1}\left(\begin{array}{l}
n_{1} \\
k_{1}
\end{array}\right)\left(\frac{n_{1}-k_{1}}{n_{1}} f\left(\frac{k_{1}}{n_{1}-1}, 0,0\right)+\frac{k_{1}}{n_{1}} f\left(\frac{k_{1}-1}{n_{1}-1}, 0,0\right)-f\left(\frac{k_{1}}{n_{1}}, 0,0\right)\right) t_{1}^{k_{1}} \\
& +\sum_{k_{2}=1}^{n_{2}-1}\left(\begin{array}{l}
n_{2} \\
k_{2}
\end{array}\right)\left(\frac{n_{2}-k_{2}}{n_{2}} f\left(0, \frac{k_{2}}{n_{2}-1}, 0\right)+\frac{k_{2}}{n_{2}} f\left(0, \frac{k_{2}-1}{n_{2}-1}, 0\right)-f\left(0, \frac{k_{2}}{n_{2}}, 0\right)\right) t_{2}^{k_{2}} \\
& +\sum_{k_{1}=1}^{n_{1}-1}\left(\begin{array}{l}
n_{3} \\
k_{3}
\end{array}\right)\left(\frac{n_{3}-k_{3}}{n_{3}} f\left(0,0, \frac{k_{3}}{n_{3}-1}\right)+\frac{k_{3}}{n_{3}} f\left(0,0, \frac{k_{3}-1}{n_{3}-1}\right)-f\left(0,0, \frac{k_{3}}{n_{3}}\right)\right) t_{3}^{k_{3}} \\
& +\sum_{k_{1}=1}^{n_{1}-1}\left(\begin{array}{l}
n_{1} \\
k_{1}
\end{array}\right)\left(\frac{n_{1}-k_{1}}{n_{1}} f\left(\frac{k_{1}}{n_{1}-1}, 1,1\right)+\frac{k_{1}}{n_{1}} f\left(\frac{k_{1}-1}{n_{1}-1}, 1,1\right)-f\left(\frac{k_{1}}{n_{1}}, 1,1\right)\right) t_{1}^{k_{1}} t_{2}^{n_{2}} t_{3}^{n_{3}} \\
& +\sum_{k_{2}=1}^{n_{2}-1}\left(\begin{array}{l}
n_{2} \\
k_{2}
\end{array}\right)\left(\frac{n_{2}-k_{2}}{n_{2}} f\left(1, \frac{k_{2}}{n_{2}-1}, 1\right)+\frac{k_{2}}{n_{2}} f\left(1, \frac{k_{2}-1}{n_{2}-1}, 1\right)-f\left(1, \frac{k_{2}}{n_{2}}, 1\right)\right) t_{1}^{n_{1}} t_{2}^{k_{2}} t_{3}^{n_{3}} \\
& +\sum_{k_{1}=1}^{n_{1}-1}\left(\begin{array}{l}
n_{3} \\
k_{3}
\end{array}\right)\left(\frac{n_{3}-k_{3}}{n_{3}} f\left(1,1, \frac{k_{3}}{n_{3}-1}\right)+\frac{k_{3}}{n_{3}} f\left(1,1, \frac{k_{3}-1}{n_{3}-1}\right)-f\left(1,1, \frac{k_{3}}{n_{3}}\right)\right) t_{1}^{n_{1}} t_{2}^{n_{2}} t_{3}^{k_{3}} \\
& +\sum_{k_{1}=1}^{n_{1}-1}\left(\begin{array}{l}
n_{1} \\
k_{1}
\end{array}\right)\left(\frac{n_{1}-k_{1}}{n_{1}} f\left(\frac{k_{1}}{n_{1}-1}, 1,0\right)+\frac{k_{1}}{n_{1}} f\left(\frac{k_{1}-1}{n_{1}-1}, 1,0\right)-f\left(\frac{k_{1}}{n_{1}}, 1,0\right)\right) t_{1}^{k_{1}} t_{2}^{n_{2}} \\
& +\sum_{k_{1}=1}^{n_{1}-1}\left(\begin{array}{l}
n_{1} \\
k_{1}
\end{array}\right)\left[\frac{n_{1}-k_{1}}{n_{1}} f\left(\frac{k_{1}}{n_{1}-1}, 0,1\right)+\frac{k_{1}}{n_{1}} f\left(\frac{k_{1}-1}{n_{1}-1}, 0,1\right)-f\left(\frac{k_{1}}{n_{1}}, 0,1\right)\right] t_{1}^{k_{1}} t_{3}^{n_{3}} \\
& +\sum_{k_{2}=1}^{n_{2}-1}\left(\begin{array}{l}
n_{2} \\
k_{2}
\end{array}\right)\left(\frac{n_{2}-k_{2}}{n_{2}} f\left(1, \frac{k_{2}}{n_{2}-1}, 0\right)+\frac{k_{2}}{n_{2}} f\left(1, \frac{k_{2}-1}{n_{2}-1}, 0\right)-f\left(1, \frac{k_{2}}{n_{2}}, 0\right)\right) t_{1}^{n_{1}} t_{2}^{k_{2}} \\
& +\sum_{k_{2}=1}^{n_{2}-1}\left(\begin{array}{l}
n_{2} \\
k_{2}
\end{array}\right)\left(\frac{n_{2}-k_{2}}{n_{2}} f\left(0, \frac{k_{2}}{n_{2}-1}, 1\right)+\frac{k_{2}}{n_{2}} f\left(0, \frac{k_{2}-1}{n_{2}-1}, 1\right)-f\left(0, \frac{k_{2}}{n_{2}}, 1\right)\right) t_{2}^{k_{2}} t_{3}^{n_{3}} \\
& +\sum_{k_{1}=1}^{n_{1}-1}\left(\begin{array}{l}
n_{3} \\
k_{3}
\end{array}\right)\left(\frac{n_{3}-k_{3}}{n_{3}} f\left(1,0, \frac{k_{3}}{n_{3}-1}\right)+\frac{k_{3}}{n_{3}} f\left(1,0, \frac{k_{3}-1}{n_{3}-1}\right)-f\left(1,0, \frac{k_{3}}{n_{3}}\right)\right) t_{1}^{n_{1}} t_{3}^{k_{3}} \\
& +\sum_{k_{1}=1}^{n_{1}-1}\left(\begin{array}{l}
n_{3} \\
k_{3}
\end{array}\right)\left(\frac{n_{3}-k_{3}}{n_{3}} f\left(0,1, \frac{k_{3}}{n_{3}-1}\right)+\frac{k_{3}}{n_{3}} f\left(0,1, \frac{k_{3}-1}{n_{3}-1}\right)-f\left(0,1, \frac{k_{3}}{n_{3}}\right)\right) t_{2}^{n_{2}} t_{3}^{k_{3}} \text {. }
\end{aligned}
$$

Then $\mathrm{F}_{n_{1}, n_{2}, n_{3}}^{4}\left(t_{1}, t_{2}, t_{3}\right)$ is positive since $f$ is convex and all the $t_{i}, i \in\{1,2,3\}$, are positive.

\section{Order of Convergence}

To provide information about the speed of convergence of multivariate Bernstein polynomials, we need the following intermediate result. This result also exists for one variable (see (Reference [1] Lemma 6.3.5)).

Lemma 4. There is a constant $C$ independent of $n_{i}$ such that, for all $\left(x_{1}, \cdots, x_{m}\right) \in[0,1]^{m}, i=1, \cdots, m$,

$$
\sum_{\left|\frac{k_{1}}{n_{1}}-x_{1}\right| \geq n_{1}^{-1 / 4}} \cdots \sum_{\left|\frac{k_{m}}{n_{m}}-x_{m}\right| \geq n_{m}^{-1 / 4}} \prod_{i=1}^{m}\left(\begin{array}{l}
n_{i} \\
k_{i}
\end{array}\right) x_{i}^{k_{i}}\left(1-x_{i}\right)^{n_{i}-k_{i}} \leq \frac{C}{\prod_{i=1}^{m} n_{i}^{3 / 2}} .
$$


Proof of Lemma 4. We will sometimes use the square brackets to draw attention to computations of certain terms.

$$
\begin{aligned}
& \sum_{\left|\frac{k_{1}}{n_{1}}-x_{1}\right| \geq n_{1}^{-1 / 4}} \cdots \sum_{\left|\frac{k_{m}}{n_{m}}-x_{m}\right| \geq n_{m}^{-1 / 4}} \prod_{i=1}^{m}\left(\begin{array}{l}
n_{i} \\
k_{i}
\end{array}\right) x_{i}^{k_{i}}\left(1-x_{i}\right)^{n_{i}-k_{i}}
\end{aligned}
$$

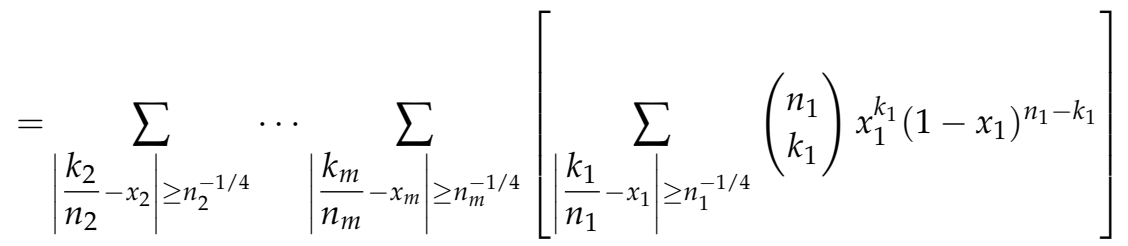

$$
\begin{aligned}
& \times \prod_{i=2}^{m}\left(\begin{array}{l}
n_{i} \\
k_{i}
\end{array}\right) x_{i}^{k_{i}}\left(1-x_{i}\right)^{n_{i}-k_{i}},
\end{aligned}
$$

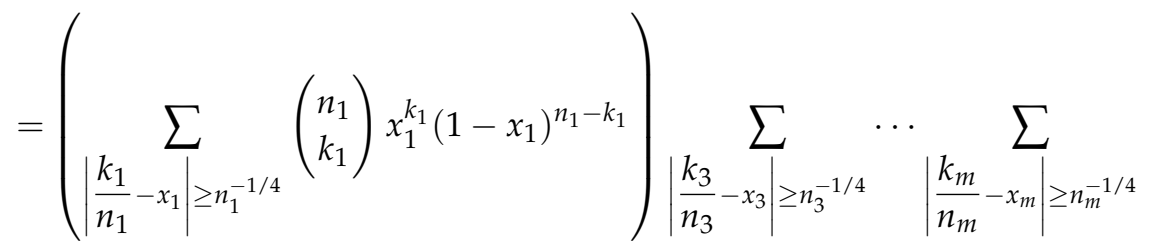

$$
\begin{aligned}
& {\left[\sum_{\left|\frac{k_{2}}{n_{2}}-x_{2}\right| \geq n_{2}^{-1 / 4}}\left(\begin{array}{l}
n_{2} \\
k_{2}
\end{array}\right) x_{2}^{k_{2}}\left(1-x_{2}\right)^{n_{2}-k_{2}}\right] \prod_{i=3}^{m}\left(\begin{array}{l}
n_{i} \\
k_{i}
\end{array}\right) x_{i}^{k_{i}}\left(1-x_{i}\right)^{n_{i}-k_{i}}}
\end{aligned}
$$

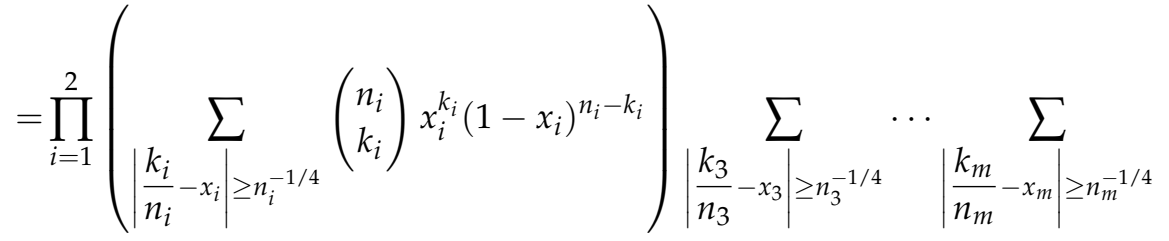

$$
\begin{aligned}
& \prod_{i=3}^{m}\left(\begin{array}{l}
n_{i} \\
k_{i}
\end{array}\right) x_{i}^{k_{i}}\left(1-x_{i}\right)^{n_{i}-k_{i}} .
\end{aligned}
$$

By repeating successively the actions with $k_{3}, k_{4}, \cdots, k_{m}$, we obtain

$$
\sum_{\left|\frac{k_{1}}{n_{1}}-x_{1}\right| \geq n_{1}^{-1 / 4}} \ldots \sum_{\left|\frac{k_{m}}{n_{m}}-x_{m}\right| \geq n_{m}^{-1 / 4}} \prod_{i=1}^{m}\left(\begin{array}{c}
n_{i} \\
k_{i}
\end{array}\right) x_{i}^{k_{i}}\left(1-x_{i}\right)^{n_{i}-k_{i}}=\prod_{i=1}^{m} \sum_{\left|\frac{k_{i}}{n_{i}}-x_{i}\right| \geq n_{i}^{-1 / 4}}\left(\begin{array}{c}
n_{i} \\
k_{i}
\end{array}\right) x_{i}^{k_{i}}\left(1-x_{i}\right)^{n_{i}-k_{i}} .
$$

Let us use the following result which can be found in References $[1,21,22]$ : There is a constant $C$ independent of a given positive integer $n$ such that for all $x$ in $[0,1]$,

$$
\sum_{\left|\frac{k}{n}-x\right| \geq n^{-1 / 4}}\left(\begin{array}{l}
n \\
k
\end{array}\right) x^{k}(1-x)^{n-k} \leq \frac{C}{n^{3 / 2}}
$$

By applying this to any $x_{i}, i=\{1, \cdots, m\}$, we have 


$$
\begin{aligned}
& \sum_{\left|\frac{k_{1}}{n_{1}}-x_{1}\right| \geq n_{1}^{-1 / 4}} \cdots \sum_{\left|\frac{k_{m}}{n_{m}}-x_{m}\right| \geq n_{m}^{-1 / 4}} \prod_{i=1}^{m}\left(\begin{array}{l}
n_{i} \\
k_{i}
\end{array}\right) x_{i}^{k_{i}}\left(1-x_{i}\right)^{n_{i}-k_{i}} \\
& \leq \prod_{i=1}^{m}\left(\frac{C_{i}}{n_{i}^{3 / 2}}\right) \text { with } C_{i} \text { constants } \\
& =\frac{C}{\prod_{i=1}^{m} n_{i}^{3 / 2}} \text { with } C \text { constant }\left(C=C_{1} \cdots C_{m}\right) \text {. }
\end{aligned}
$$

Remark 2. Lemma 4 is still valid if the summation is not on all the $k_{i}, 1 \leq i \leq m$ but on some of them. In this case, the upper bound will contain only terms involved in the summation.

The following Theorem will help us to conclude on the order of convergence of our Bernstein polynomials with several variables, see References [22,36].

Theorem 6. Let $f\left(x_{1}, \cdots, x_{m}\right)$ be bounded in $[0,1]^{m}$ and let $\left(a_{1}, \cdots, a_{m}\right)$ be a point of $[0,1]^{m}$ at which $f^{(2) \cdots(2)}\left(a_{1}, \cdots, a_{m}\right)$ exists and is continuous. Then,

$$
\lim _{n_{j} \rightarrow \infty, 1 \leq j \leq m}\left[B_{n_{1}, \cdots, n_{m}}\left(f ; a_{1}, \cdots, a_{m}\right)-f\left(a_{1}, \cdots, a_{m}\right)-\frac{1}{2} \sum_{j=1}^{m} \frac{a_{j}\left(1-a_{j}\right)}{n_{j}} \frac{\partial^{2} f}{\partial x_{j}^{2}}\left(a_{1}, \cdots, a_{m}\right)\right]=0,
$$

and

$$
\lim _{N \rightarrow \infty} N\left[B_{N}, \cdots, N\left(f ; a_{1}, \cdots, a_{m}\right)-f\left(a_{1}, \cdots, a_{m}\right)\right]=\frac{1}{2} \sum_{i=1}^{m} a_{i}\left(1-a_{i}\right) \frac{\partial^{2} f}{\partial x_{i}^{2}}\left(a_{1}, \cdots, a_{m}\right) .
$$

Proof of Theorem 6. Since $f^{(2) \cdots(2)}\left(x_{1}, \cdots, x_{m}\right)$ exists and is continuous at $\left(a_{1}, \cdots, a_{m}\right)$, then using Taylor expansion of order two

$$
\begin{gathered}
f\left(x_{1}, \cdots, x_{m}\right) \\
=f\left(a_{1}, \cdots, a_{m}\right)+\sum_{j=1}^{m} \frac{\partial f}{\partial x_{j}}\left(a_{1}, \cdots, a_{m}\right)\left(x_{j}-a_{j}\right)+\frac{1}{2}\left(\sum_{j=1}^{m} \frac{\partial^{2} f}{\partial x_{j}^{2}}\left(a_{1}, \cdots, a_{m}\right)\left(x_{j}-a_{j}\right)^{2}\right. \\
\left.\quad+\sum_{i, j=1, i \neq j}^{m} \frac{\partial^{2} f}{\partial x_{i} \partial x_{j}}\left(a_{1}, \cdots, a_{m}\right)\left(x_{i}-a_{i}\right)\left(x_{j}-a_{j}\right)\right)+s\left(x_{1}, \cdots, x_{m}\right) \sum_{j=1}^{m}\left(x_{j}-a_{j}\right)^{2},
\end{gathered}
$$

where $\lim _{\left(x_{1}, \cdots, x_{m}\right) \rightarrow\left(a_{1}, \cdots, a_{m}\right)} s\left(x_{1}, \cdots, x_{m}\right)=0$. Set $\left(x_{1}, \cdots, x_{m}\right)=\left(\frac{k_{1}}{n_{1}}, \cdots, \frac{k_{m}}{n_{m}}\right)$, multiplying both sides by $\prod_{i=1}^{m}\left(\begin{array}{l}n_{i} \\ k_{i}\end{array}\right) a_{i}^{k_{i}}\left(1-a_{i}\right)^{n_{i}-k_{i}}$ and sum from $k_{i}=0$ to $k_{i}=n_{i}$, for all $i=1, \cdots, m$, we obtain 


$$
\begin{aligned}
& B_{n_{1}, \cdots, n_{m}}\left(f ; a_{1}, \cdots, a_{m}\right) \\
& =f\left(a_{1}, \cdots, a_{m}\right)+\sum_{j=1}^{m} \frac{\partial f}{\partial x_{j}}\left(a_{1}, \cdots, a_{m}\right) \sum_{k_{1}=0}^{n_{1}} \cdots \sum_{k_{m}=0}^{n_{m}}\left(\frac{k_{j}}{n_{j}}-a_{j}\right) \prod_{i=1}^{m}\left(\begin{array}{l}
n_{i} \\
k_{i}
\end{array}\right) a_{i}^{k_{i}}\left(1-a_{i}\right)^{n_{i}-k_{i}} \\
& +\frac{1}{2} \sum_{j=1}^{m} \frac{\partial^{2} f}{\partial x_{j}^{2}}\left(a_{1}, \cdots, a_{m}\right) \sum_{k_{1}=0}^{n_{1}} \cdots \sum_{k_{m}=0}^{n_{m}}\left(\frac{k_{j}}{n_{j}}-a_{j}\right)^{2} \prod_{i=1}^{m}\left(\begin{array}{c}
n_{i} \\
k_{i}
\end{array}\right) a_{i}^{k_{i}}\left(1-a_{i}\right)^{n_{i}-k_{i}} \\
& +\frac{1}{2} \sum_{i, j=1, i \neq j}^{m} \frac{\partial^{2} f}{\partial x_{i} \partial x_{j}}\left(a_{1}, \cdots, a_{m}\right) \sum_{k_{1}=0}^{n_{1}} \cdots \sum_{k_{m}=0}^{n_{m}}\left(\frac{k_{i}}{n_{i}}-a_{i}\right)\left(\frac{k_{j}}{n_{j}}-a_{j}\right) \prod_{l=1}^{m}\left(\begin{array}{l}
n_{l} \\
k_{l}
\end{array}\right) a_{l}^{k_{l}}\left(1-a_{l}\right)^{n_{l}-k_{l}} \\
& +\sum_{k_{1}=0}^{n_{1}} \cdots \sum_{k_{m}=0}^{n_{m}} s\left(\frac{k_{1}}{n_{1}}, \cdots, \frac{k_{m}}{n_{m}}\right) \sum_{j=1}^{m}\left(\frac{k_{j}}{n_{j}}-a_{j}\right)^{2} \prod_{i=1}^{m}\left(\begin{array}{l}
n_{i} \\
k_{i}
\end{array}\right) a_{i}^{k_{i}}\left(1-a_{i}\right)^{n_{i}-k_{i}} \\
& =f\left(a_{1}, \cdots, a_{m}\right)+\sum_{j=1}^{m} \frac{\partial f}{\partial x_{j}}\left(a_{1}, \cdots, a_{m}\right)\left(\sum_{k_{j}=0}^{n_{j}}\left(\frac{k_{j}}{n_{j}}-a_{j}\right)\left(\begin{array}{l}
n_{j} \\
k_{j}
\end{array}\right) a_{j}^{k_{j}}\left(1-a_{j}\right)^{n_{j}-k_{j}}\right) \\
& \times \sum_{k_{1}=0,1 \neq j}^{n_{1}} \ldots \sum_{k_{m}=0, m \neq j}^{n_{m}} \prod_{i=1, i \neq j}^{m}\left(\begin{array}{c}
n_{i} \\
k_{i}
\end{array}\right) a_{i}^{k_{i}}\left(1-a_{i}\right)^{n_{i}-k_{i}}+\frac{1}{2} \sum_{j=1}^{m} \frac{\partial^{2} f}{\partial x_{j}^{2}}\left(a_{1}, \cdots, a_{m}\right) \\
& \times\left(\sum_{k_{j}=0}^{n_{j}}\left(\frac{k_{j}}{n_{j}}-a_{j}\right)^{2}\left(\begin{array}{l}
n_{j} \\
k_{j}
\end{array}\right) a_{j}^{k_{j}}\left(1-a_{j}\right)^{n_{j}-k_{j}}\right) \sum_{k_{1}=0,1 \neq j}^{n_{1}} \ldots \sum_{k_{m}=0, m \neq j}^{n_{m}} \prod_{i=1, i \neq j}^{m}\left(\begin{array}{l}
n_{i} \\
k_{i}
\end{array}\right) a_{i}^{k_{i}}\left(1-a_{i}\right)^{n_{i}-k_{i}} \\
& +\frac{1}{2} \sum_{i, j=1, i \neq j}^{m} \frac{\partial^{2} f}{\partial x_{i} \partial x_{j}}\left(a_{1}, \cdots, a_{m}\right)\left(\sum_{k_{i}=0}^{n_{i}}\left(\frac{k_{i}}{n_{i}}-a_{i}\right)\left(\begin{array}{l}
n_{i} \\
k_{i}
\end{array}\right) a_{i}^{k_{i}}\left(1-a_{i}\right)^{n_{i}-k_{i}}\right)\left(\sum_{k_{j}=0}^{n_{j}}\left(\frac{k_{j}}{n_{j}}-a_{j}\right)\left(\begin{array}{l}
n_{j} \\
k_{j}
\end{array}\right)\right. \\
& \left.\times a_{j}^{k_{j}}\left(1-a_{j}\right)^{n_{j}-k_{j}}\right) \sum_{k_{1}=0,1 \neq i, j}^{n_{1}} \ldots \sum_{k_{m}=0, m \neq i, j}^{n_{m}} \prod_{l=1, l \neq i, j}^{m}\left(\begin{array}{l}
n_{l} \\
k_{l}
\end{array}\right) a_{l}^{k_{l}}\left(1-a_{l}\right)^{n_{l}-k_{l}} \\
& +\sum_{j=1}^{m}\left(\sum_{k_{1}=0}^{n_{1}} \cdots \sum_{k_{m}=0}^{n_{m}} s\left(\frac{k_{1}}{n_{1}}, \cdots, \frac{k_{m}}{n_{m}}\right)\left(\frac{k_{j}}{n_{j}}-a_{j}\right)^{2} \prod_{i=1}^{m}\left(\begin{array}{c}
n_{i} \\
k_{i}
\end{array}\right) a_{i}^{k_{i}}\left(1-a_{i}\right)^{n_{i}-k_{i}}\right) .
\end{aligned}
$$

Let us use the following results from P. J. Davis [1] which are valid for all $x \in[0,1]$ :

$$
\left\{\begin{array}{l}
\sum_{k=0}^{n}\left(\begin{array}{l}
n \\
k
\end{array}\right)\left(\frac{k}{n}-x\right) x^{k}(1-x)^{n-k}=0 \\
\sum_{k=0}^{n}\left(\begin{array}{l}
n \\
k
\end{array}\right)\left(\frac{k}{n}-x\right)^{2} x^{k}(1-x)^{n-k}=\frac{x(1-x)}{n} ; \\
\sum_{k=0}^{n}\left(\begin{array}{l}
n \\
k
\end{array}\right)\left(\frac{k}{n}-x\right)^{3} x^{k}(1-x)^{n-k}=x(1-x) \frac{-2 x+1}{n^{2}} ; \\
\sum_{k=0}^{n}\left(\begin{array}{l}
n \\
k
\end{array}\right)\left(\frac{k}{n}-x\right)^{4} x^{k}(1-x)^{n-k}=x(1-x) \frac{(3 n-6) x(1-x)+1}{n^{3}} .
\end{array}\right.
$$

The second equation in system (135) can also be found in (Reference [33] Corollary 5.3). Hence, with Equations (31) and (135), we have

$$
\begin{aligned}
& B_{n_{1}, \cdots, n_{m}}\left(f ; a_{1}, \cdots, a_{m}\right) \\
= & f\left(a_{1}, \cdots, a_{m}\right)+\sum_{j=1}^{m} \frac{a_{j}\left(1-a_{j}\right)}{2 n_{j}} \frac{\partial^{2} f}{\partial x_{j}^{2}}\left(a_{1}, \cdots, a_{m}\right) \\
& +\sum_{j=1}^{m}\left(\sum_{k_{1}=0}^{n_{1}} \cdots \sum_{k_{m}=0}^{n_{m}} s\left(\frac{k_{1}}{n_{1}}, \cdots, \frac{k_{m}}{n_{m}}\right)\left(\frac{k_{j}}{n_{j}}-a_{j}\right)^{2} \prod_{i=1}^{m}\left(\begin{array}{c}
n_{i} \\
k_{i}
\end{array}\right) a_{i}^{k_{i}}\left(1-a_{i}\right)^{n_{i}-k_{i}}\right) .
\end{aligned}
$$


Let

$$
S=\sum_{j=1}^{m}\left(\sum_{k_{1}=0}^{n_{1}} \cdots \sum_{k_{m}=0}^{n_{m}} s\left(\frac{k_{1}}{n_{1}}, \cdots, \frac{k_{m}}{n_{m}}\right)\left(\frac{k_{j}}{n_{j}}-a_{j}\right)^{2} \prod_{i=1}^{m}\left(\begin{array}{l}
n_{i} \\
k_{i}
\end{array}\right) a_{i}^{k_{i}}\left(1-a_{i}\right)^{n_{i}-k_{i}}\right)
$$

and $\varepsilon>0$. We can find $n_{i}, i=1, \cdots, m$ sufficiently large such that $\left|x_{i}-a_{i}\right|<\frac{1}{n_{i}^{1 / 4}}$ implies $\left|s\left(x_{1}, \cdots, x_{m}\right)\right| \leq \varepsilon$ since $\lim _{\left(x_{1}, \cdots, x_{m}\right) \rightarrow\left(a_{1}, \cdots, a_{m}\right)} s\left(x_{1}, \cdots, x_{m}\right)=0$. Let us consider the set $E=\prod_{i=1}^{m}\left\{0, \cdots, n_{i}\right\}$, and for $j=1, \cdots, m$, define the sets

$$
\Omega_{j}=\left\{k_{j} \in\left\{0, \cdots, n_{j}\right\}:\left|\frac{k_{j}}{n_{j}}-a_{j}\right|<\frac{1}{n_{j}^{1 / 4}}\right\} \text { and } F=E \backslash\left(\Omega_{1} \times \cdots \times \Omega_{m}\right) .
$$

Then $F=\bigcup_{k=1}^{m} F_{k}$, with $F_{k}=\left\{\prod_{i=1}^{m} \Omega_{i k}^{\left[\alpha_{i k}\right]} \in F: \alpha_{i k} \in\{0,1\}, \quad \sum_{i=1}^{m} \alpha_{i k}=k\right\}$, where $\Omega_{i k}^{\left[\alpha_{i k}\right]}=$ $\left\{\begin{array}{ll}\Omega_{i} & \text { if } \alpha_{i k}=0 \\ \Omega_{i}^{c} & \text { if } \alpha_{i k}=1\end{array}\right.$ and $\Omega_{i}^{c}=\left\{k_{i} \in\left\{0, \cdots, n_{i}\right\}:\left|\frac{k_{i}}{n_{i}}-x_{i}\right| \geq \frac{1}{n_{i}^{1 / 4}}\right\}$. For $A_{k}=\prod_{i=1}^{m} \Omega_{i k}^{\left[\alpha_{i k}\right]} \in F_{k}$, $k=1, \cdots, m$, let us also define $I_{A_{k}}=\left\{i \in\{1, \cdots, m\}: \alpha_{i k}=1\right\}$ for $k=1, \cdots, m$ (that means $\left.\operatorname{card}\left(I_{A_{k}}\right)=k \geq 1\right)$. We have

$$
\begin{aligned}
& |S| \leq \sum_{j=1}^{m}\left(\sum_{\Omega_{1}} \cdots \sum_{\Omega_{m}}\left|s\left(\frac{k_{1}}{n_{1}}, \cdots, \frac{k_{m}}{n_{m}}\right)\right|\left(\frac{k_{j}}{n_{j}}-a_{j}\right)^{2} \prod_{i=1}^{m}\left(\begin{array}{l}
n_{i} \\
k_{i}
\end{array}\right) a_{i}^{k_{i}}\left(1-a_{i}\right)^{n_{i}-k_{i}}\right. \\
& \left.+\sum_{F}\left|s\left(\frac{k_{1}}{n_{1}}, \cdots, \frac{k_{m}}{n_{m}}\right)\right|\left(\frac{k_{j}}{n_{j}}-a_{j}\right)^{2} \prod_{i=1}^{m}\left(\begin{array}{l}
n_{i} \\
k_{i}
\end{array}\right) a_{i}^{k_{i}}\left(1-a_{i}\right)^{n_{i}-k_{i}}\right) \\
& \leq \varepsilon \sum_{j=1}^{m}\left(\sum_{k_{1}=0}^{n_{1}} \cdots \sum_{k_{m}=0}^{n_{m}}\left(\frac{k_{j}}{n_{j}}-a_{j}\right)^{2} \prod_{i=1}^{m}\left(\begin{array}{l}
n_{i} \\
k_{i}
\end{array}\right) a_{i}^{k_{i}}\left(1-a_{i}\right)^{n_{i}-k_{i}}\right) \\
& \left.+M \sum_{l=1}^{m} \sum_{A_{l} \in F_{l}}\left(\prod_{i=1}^{m}\left(\begin{array}{l}
n_{i} \\
k_{i}
\end{array}\right) a_{i}^{k_{i}}\left(1-a_{i}\right)^{n_{i}-k_{i}}\right]\right) \quad\left(\text { since } F=\bigcup_{l=1}^{m} F_{l}\right) \\
& \text { where } M=\sup _{\left(x_{1}, \cdots, x_{m}\right) \in[0,1]^{m}}\left|s\left(x_{1}, \cdots, x_{m}\right)\right| \sum_{j=1}^{m}\left(x_{j}-a_{j}\right)^{2} \\
& \leq \varepsilon \sum_{j=1}^{m}\left(\left(\sum_{k_{j}=0}^{n_{j}}\left(\frac{k_{j}}{n_{j}}-a_{j}\right)^{2}\left(\begin{array}{l}
n_{j} \\
k_{j}
\end{array}\right) a_{j}^{k_{j}}\left(1-a_{j}\right)^{n_{j}-k_{j}}\right) \sum_{k_{1}=0,1 \neq j}^{n_{1}} \ldots\right. \\
& \left.\sum_{k_{m}=0, m \neq j}^{n_{m}} \prod_{i=1, i \neq j}^{m}\left(\begin{array}{c}
n_{i} \\
k_{i}
\end{array}\right) a_{i}^{k_{i}}\left(1-a_{i}\right)^{n_{i}-k_{i}}\right)+M \sum_{l=1}^{m} \sum_{A_{l} \in F_{l}} \prod_{i \in I_{A_{l}}} \frac{C_{i}}{n_{i}^{3 / 2}}
\end{aligned}
$$

with $C_{i}$ constants (we bounded the summation on $F$ using Equation (129) and the same procedure as we have used to show (55) in Theorem 2. Hence, 


$$
\begin{aligned}
|S| \leq & \varepsilon \sum_{j=1}^{m}\left(\sum_{k_{j}=0}^{n_{j}}\left(\frac{k_{j}}{n_{j}}-a_{j}\right)^{2}\left(\begin{array}{c}
n_{j} \\
k_{j}
\end{array}\right) a_{j}^{k_{j}}\left(1-a_{j}\right)^{n_{j}-k_{j}}\right)+M \sum_{l=1}^{m} \sum_{A_{l} \in F_{l}} \prod_{i \in I_{A_{l}}} \frac{C}{n^{3 / 2}} \\
& \quad\left(\text { using }(31), \text { and } n=\min \left\{n_{j}, 1 \leq j \leq m\right\} \leq n_{i}, i=1, \cdots, m \text { and } C=\max \left(C_{i}: i=1, \cdots, m\right)\right) \\
\leq & \left.\varepsilon \sum_{j=1}^{m}\left(\frac{a_{j}\left(1-a_{j}\right)}{n_{j}}\right)+M \sum_{l=1}^{m} \sum_{A_{l} \in F_{l}} \frac{C^{l}}{n^{3 l / 2}} \quad \text { (using Equation }(135) \text { and card }\left(I_{A_{l}}\right)=l\right) \\
\leq & \frac{\varepsilon}{n} \sum_{j=1}^{m}\left(a_{j}\left(1-a_{j}\right)\right)+\frac{1}{n} \sum_{l=1}^{m} \sum_{A_{l} \in F_{l}} \frac{M \times C^{l}}{n^{1 / 2}} \quad\left(\text { since } n^{3 l / 2}>n^{3 / 2}\right) .
\end{aligned}
$$

For $n_{1}, \cdots, n_{m}$ sufficiently large (that means $n$ sufficiently large), we have $\sum_{l=1}^{m} \sum_{A_{l} \in F_{l}} \frac{M \times C^{l}}{n^{1 / 2}}<\varepsilon$. This implies

$$
|S| \leq \frac{\varepsilon}{n}\left(1+\sum_{j=1}^{m} a_{j}\left(1-a_{j}\right)\right)
$$

Therefore,

$$
\begin{aligned}
& \left|B_{n_{1}, \cdots, n_{m}}\left(f ; a_{1}, \cdots, a_{m}\right)-f\left(a_{1}, \cdots, a_{m}\right)-\sum_{j=1}^{m} \frac{a_{j}\left(1-a_{j}\right)}{2 n_{j}} \frac{\partial^{2} f}{\partial x_{j}^{2}}\left(a_{1}, \cdots, a_{m}\right)\right| \\
= & |S| \leq \frac{\varepsilon}{n}\left(1+\sum_{j=1}^{m} a_{j}\left(1-a_{j}\right)\right) .
\end{aligned}
$$

Since $\varepsilon$ is arbitrary, we have

$$
\lim _{n_{j} \rightarrow \infty, 1 \leq j \leq m}\left[B_{n_{1}, \cdots, n_{m}}\left(f ; a_{1}, \cdots, a_{m}\right)-f\left(a_{1}, \cdots, a_{m}\right)-\sum_{j=1}^{m} \frac{a_{j}\left(1-a_{j}\right)}{2 n_{j}} \frac{\partial^{2} f}{\partial x_{j}^{2}}\left(a_{1}, \cdots, a_{m}\right)\right]=0 .
$$

Remark 3. By taking $n_{i}=N, 1 \leq i \leq m$, in Theorem 6, we have

$$
\lim _{N \rightarrow \infty} N\left(B_{N}, \cdots, N\left(f ; a_{1}, \cdots, a_{m}\right)-f\left(a_{1}, \cdots, a_{m}\right)\right)=\frac{1}{2} \sum_{i=1}^{m} a_{i}\left(1-a_{i}\right) \frac{\partial^{2} f}{\partial x_{i}^{2}}\left(a_{1}, \cdots, a_{m}\right) .
$$

From the previous Remark, one can deduce that the order of convergence of Bernstein polynomials with several variables for a bounded function $f\left(x_{1}, \cdots, x_{m}\right),\left(x_{1}, \cdots, x_{m}\right) \in[0,1]^{m}$, in some neighborhood of $\left(a_{1}, \cdots, a_{m}\right) \in[0,1]^{m}$ included in $[0,1]^{m}$, is one, provided that $f^{(2)}\left(a_{1}, \cdots, a_{m}\right)$ exists. That is less than Taylor's order of convergence.

\section{Conclusions}

Our concern here was the multivariate Bernstein polynomials given by Equation (6) which we recall here

$$
B_{n_{1}, \cdots, n_{m}}\left(f ; x_{1}, \cdots, x_{m}\right)=\sum_{k_{1}=0}^{n_{1}} \cdots \sum_{k_{m}=0}^{n_{m}} f\left(\frac{k_{1}}{n_{1}}, \cdots, \frac{k_{m}}{n_{m}}\right) \prod_{i=1}^{m}\left(\begin{array}{l}
n_{i} \\
k_{i}
\end{array}\right) x_{i}^{k_{i}}\left(1-x_{i}\right)^{n_{i}-k_{i}},
$$

and which is a generalization in several variables of the univariate Bernstein polynomials given by (1) 


$$
B_{n}(f ; x)=\sum_{k=0}^{n} f\left(\frac{k}{n}\right)\left(\begin{array}{l}
n \\
k
\end{array}\right) x^{k}(1-x)^{n-k} .
$$

We extended the beautiful properties of the above mentioned univariate Bernstein polynomials by generalizing them for functions of several real variables: uniform convergence, uniform convergence of the derivatives, order of convergence, monotonicity, fixed sign for the $p$-th derivative, and deduction of the upper and lower bounds of the Bernstein polynomials from those of the corresponding functions. To achieve that, we first recalled not only these properties for univariate Bernstein polynomials, but also the intermediate properties used to prove them (see Equations (11)-(16)). Then, we extended these intermediate properties for the univariate case to obtain the generalised intermediate properties for the multivariate case given by (6) (see (20)-(26)). Finally, using those generalized intermediate properties in combination with some others tools related to functions with several variables, we got the generalization of these beautiful properties of the univariate Bernstein polynomials to multivariate Bernstein polynomials given by Equation (6).

More precisely, our contributions in this paper related to this form of multivariate Bernstein polynomials can be summarized as follows:

1. deriving the expression of these multivariate Bernstein polynomials in terms of differences (similar to the case of one variable in References [1,15]);

2. establishing the pointwise and uniform convergence of these Bernstein polynomials with several variables (a generalization of the case of two variables treated in (Reference [28] Lemma 1));

3. deriving the expression of the $\left(p_{1}, \cdots, p_{m}\right)$-th derivative of these multivariate Bernstein polynomials of order $\left(n_{1}+p_{1}, \cdots, n_{m}+p_{m}\right)$-th in terms of $\left(p_{1}, \cdots, p_{m}\right)$-th differences of the corresponding functions (similar to the case of one variable in References [1,15]);

4. proving the monotonicity, the fixed sign of the $\left(p_{1}, \cdots, p_{m}\right)$-th derivative, the convexity, and the upper and lower bounds of these multivariate Bernstein polynomials from those of the corresponding functions (similar to the case of one variable in References [1,31]);

5. establishing the pointwise and uniform convergence of the $\left(p_{1}, \cdots, p_{m}\right)$-th derivative of these multivariate Bernstein polynomials (a generalization of the case of two variables treated in (Reference [35] Theorem of Kingsley) and (Reference [28] Theorem 1));

6. generalizing the inequality related to the decreasing of the sequence of Bernstein's polynomials with one variable (see References [1,31]) when $f$ is a convex function;

7. proving that the order of convergence of these Bernstein polynomials with several variables is one.

With these new results, we have proved that the conclusion made by P.J. Davis in Reference [1] for Bernstein polynomials of one variable is true for the case of several variables, that is, the Bernstein polynomials of several variables yield smooth approximants, but their convergence is very slow.

Author Contributions: The conceptualization and the supervision were done by the first author (M.F.). The formal analysis and the investigation were done by the second author (M.M.W.). All authors have read and agreed to the published version of the manuscript.

Funding: The work of these two authors was initially funded by the African Institute for Mathematical SciencesCameroon, during the academic year 2013/2014.

Acknowledgments: The authors would like to acknowledge the support of the two institutions where this work has been carried out: The Mathematics Institute of the University of Kassel in Germany and the African Institute for Mathematical Sciences in Cameroon.

Conflicts of Interest: The authors declare that they have no known competing financial interests or personal relation-ships that could have appeared to influence the work reported in this paper. 


\section{References}

1. Davis, P.J. Interpolation and Approximation; Dover Publications: New York, NY, USA, 1975.

2. Kim, T.; Jang, L.-C.; Yi, H. A note on the modified q-Bernstein polynomials. Discret. Dyn. Nat. Soc. 2010. [CrossRef]

3. Lu, W.-M.; Zhang, L. Pointwise approximation theorems for combinations of Bernstein polynomials with inner singularities. Appl. Math. 2010, 2. [CrossRef]

4. Simsek, Y.; Acikgoz, M. A new generating function of (q-)Bernstein-type polynomials and their interpolation function. Abstr. Appl. Anal. 2010. [CrossRef]

5. Xie, L.S. Pointwise approximation theorems for combinations and derivatives of Bernstein polynomials. Acta Math. Sin. 2005, 21, 1241-1248. [CrossRef]

6. Xie, L. Pointwise simultaneous approximation by combination of Bernstein operators. J. Approx. Theory 2005, 137, 1-21. [CrossRef]

7. Yu, A.; Veretennikova, E.V. On partial derivatives of multivariate Bernstein polynomials. Sib. Adv. Math. 2016, 26, 294-305.

8. Gal, S.G.; Anastassiou, G.A. Shape-Preserving Approximation by Real and Complex Polynomials; Birkhäuser: Boston, MA, USA, 2010.

9. Peña, J.M. Shape Preserving Representation in Computer-Aided Geometric Design; Nova Science Publishers, Inc.: New York, NY, USA, 1999.

10. Occorsio, D. Some new properties of generalized Bernstein polynomials. Stud. Univ. Babes-Bolyai Math. 2011, $56,147-160$.

11. Costabile, F.; Gualtieri, M.I.; Serra, S. Asymptotic expansion and extrapolation for Bernstein Polynomials with applications. BIT Numer. Math. 1996, 36, 676-687. [CrossRef]

12. Jafarian, A.; Nia, S.M.; Golmankhaneh, A.K.; Baleanu, D. On Bernstein Polynomials Method to the System of Abel Integral Equations. Abstr. Appl. Anal. 2014. [CrossRef]

13. Tucker, D.H. A note on Bernstein polynomial type approximations. Proc. Am. Math. Soc. 1967, 18, 492-494. [CrossRef]

14. Doha, E.H.; Bhrawy, A.H.; Saker, M.A. Integrals of Bernstein polynomials: An application for the solution of high even-order differential equations. Appl. Math. Lett. 2011, 24, 559-565. [CrossRef]

15. Phillips, G.M. Interpolation and Approximation by Polynomials; Springer: New York, NY, USA, 2003.

16. Gzyl, H.; Palacios, J.L. On the Approximation Properties of Bernstein Polynomials via Probabilistic Tools. Boletín de la Asociación Matemática Venez. 2003, 10, 5-13.

17. Alshbool, M.H.T.; Hashim, I. Multistage Bernstein polynomials for the solutions of the Fractional Order Stiff Systems. J. King Saud Univ. Sci. 2016, 28, 280-285. [CrossRef]

18. Kadison, R.V.; Liu, Z. Bernstein Polynomials and Approximation. 2018. Available online: https://www.math. upenn.edu/kadison/bernstein.pdf (accessed on 30 April 2020).

19. Dalmo, R. Matrix factorization of multivariate Bernstein polynomials. Int. J. Pure Appl. Math. 2015, 103, 749-780. [CrossRef]

20. Dinghas, A. Über einige Identitäten vom Bernsteinschen Typus. Det Koneglige Norske Videnskabers Selskab. 1951, 24, 96-97.

21. Duchoň, M. A generalized Bernstein Approximation theorem. Tatra Mt. Math. Publ. 2011, 49, 99-109. [CrossRef]

22. Lorentz, G.G. Bernstein Polynomials; American Mathematical Soc.: New York, NY, USA, 2013.

23. Bayad, A.; Kim, T.; Rim, S.-H. Bernstein Polynomials on Simplex. 2011. Available online: https://arxiv.org/pdf/ 1106.2482.pdf (accessed on 30 April 2020).

24. Campiti, M.; Raşa, I. Extrapolation Properties of Multivariate Bernstein Polynomials. Mediterr. J. Math. 2019, 16, 109. [CrossRef]

25. Derriennic, M.-M. On multivariate Approximation by Bernstein-Type polynomials. J. Approx. Theory 1985, 45, 155-166. [CrossRef] 
26. Ding, C.; Cao, F. K-Functionals and multivariate Bernstein polynomials. J. Approx. Theory 2008, 155, $125-135$. [CrossRef]

27. Jetter, K.; Stöckler, J. An identity for multivariate Bernstein polynomials. Comput. Aided Geom. Des. 2003, 20, 563-577. [CrossRef]

28. Martinez, F.L. Some properties of two-dimensional Bernstein polynomials. J. Approx. Theory 1989, 59, 300-306. [CrossRef]

29. Heitzinger, C. Simulation and Inverse Modeling of Semiconductor Manufacturing Processes; Technische Universität Wien: Vienna, Australia, 2002.

30. Ditzian, Z. Direct estimate for Bernstein polynomials. J. Approx. Theory 1994, 79, 165-166. [CrossRef]

31. Sauer, T. Multivariate Bernstein polynomials and convexity. Comput. Aided Geom. Des. 1991, 8, 465-478. [CrossRef]

32. Popoviciu, T. Sur l'approximation des fonctions convexes d'ordre supérieur. Bull. Mathématique De La Société Roumaine Des Sci. 1941, 43, 85-141.

33. Fellhauer, A. Approximation of Smooth Functions Using Bernstein Polynomials in Multiple Variables. 2016. Available online: https:/ / arxiv.org/pdf/1609.01940.pdf (accessed on 30 April 2020).

34. Acikgoz, M.; Araci, S. A new approach to modified q-Bernstein polynomials for functions of two variables with their generating and interpolation functions. Turk. J. Anal. Number Theory 2012. [CrossRef]

35. Kingsley, E.H. Bernstein polynomials for functions of two variables of class $C^{(k)}$. Proc. Am Math. Soc. 1951, 2, 64-71.

36. Voronowskaja, E. Détermination de la forme asymptotique d'approximation des fonctions par les polynomes de M. Bernstein. C. R. Acad. Sci. URSS 1932, 79, 79-85.

37. Agrawal, P.N. Inverse theorem in simultaneous approximation by Micchelli combination of Bernstein polynomials. Demonstr. Math. 1994, 27, 501-510. [CrossRef]

38. Bernstein, S.N. Complément à l'article de E. Voronowskaja: The asymptotic behavior of the approximation of functions by their Bernstein polynomials. C. R. Acad. Sci. URSS 1932, 86-92.

39. Butzer, P.L. Linear combinations of Bernstein polynomials. Can. J. Math. 1953, 5, 559-567. [CrossRef]

40. Guo, S.; Yue, S.; Li, C.; Yang, G.; Sun, Y. A Pointwise approximation theorem for linear combinations of Bernstein polynomials. Abstr. Appl. Anal. 1996, 1. [CrossRef]

41. WooD, B. Uniform approximation by linear combinations of Bernstein-Type polynomials. J. Approx. Theory 1984, 41, 51-55. [CrossRef]

42. Acikgoz, M.; Sen, E.; Araci, S. q-Bernstein-Type polynomials for functions of two variables with their generating and interpolation functions. Turk. J. Anal. Number Theory 2013, 1, 36-42. [CrossRef]

43. Oruç, H.; Tuncer, N. On the q-Bernstein polynomials. In Algorithms for Approximation IV, The Proceedings of the 2001 International Symposium, Queensgate, Huddersfield, UK, 16-20 July 2001; University of Huddersfield: Huddersfield, UK, 2002.

44. Phillips, G.M. On generalized Bernstein polynomials. Numer. Anal. 1996, 263-269. [CrossRef]

45. Vishwakarma, D.K.; Kumar, A.A.; Kumar, A. Multivariate q-Bernstein-Schurer-Kantorovich operators. J. Math. Syst. Sci. 2016, 6, 234-241. [CrossRef]

46. Bernstein, S.N. Démonstration du théorème de Weierstrass fondée sur le calcul de probabilités. Commun. Kharkov Math. Soc. 1913, 13, 1-2.

47. Wouodjié, M.M. On Multivariate Bernstein Polynomials. Master's Thesis, African Institute for Mathematical Sciences, Limbé, Cameroon, 2014. Available online: http://www.mathematik.uni-kassel.de/ merlin/Merlin_ Master_Thesis_AIMS_Cameroon.pdf (accessed on 30 April 2020).

48. Reimer, M. Multivariate Polynomial Approximation; Birkhäuserc: Basel, Switzerland, 2012. 